University of Louisville

ThinkIR: The University of Louisville's Institutional Repository

8-2018

\title{
Combination oral immunotherapy with IL-10 and IL-12 effectively treats colon tumors via synergistic effects on IL-17 producing $\mathrm{T}$ cells and colon epithelial barrier integrity.
}

Neal Bhutiani

University of Louisville

Follow this and additional works at: https://ir.library.louisville.edu/etd

Part of the Medical Immunology Commons

\section{Recommended Citation}

Bhutiani, Neal, "Combination oral immunotherapy with IL-10 and IL-12 effectively treats colon tumors via synergistic effects on IL-17 producing T cells and colon epithelial barrier integrity." (2018). Electronic Theses and Dissertations. Paper 3013.

https://doi.org/10.18297/etd/3013

This Doctoral Dissertation is brought to you for free and open access by ThinkIR: The University of Louisville's Institutional Repository. It has been accepted for inclusion in Electronic Theses and Dissertations by an authorized administrator of ThinkIR: The University of Louisville's Institutional Repository. This title appears here courtesy of the author, who has retained all other copyrights. For more information, please contact thinkir@louisville.edu. 


\title{
COMBINATION ORAL IMMUNOTHERAPY WITH IL-10 AND IL-12 EFFECTIVELY TREATS COLON TUMORS VIA SYNERGISTIC EFFECTS ON IL-17 PRODUCING T CELLS AND COLON EPITHELIAL BARRIER INTEGRITY
}

\section{By}

Neal Bhutiani

B.S., Duke University, 2009

M.D., Johns Hopkins University School of Medicine, 2013

\author{
A Dissertation \\ Submitted to the Faculty of the \\ School of Medicine of the University of Louisville \\ in Partial Fulfillment of the Requirements \\ for the Degree of
}

Doctor of Philosophy in Microbiology and Immunology

Department of Microbiology and Immunology University of Louisville

Louisville, Kentucky 
Copyright 2018 by Neal Bhutiani

All rights reserved 

COMBINATION ORAL IMMUNOTHERAPY WITH IL-10 AND IL-12 EFFECTIVELY TREATS COLON TUMORS VIA SYNERGISTIC EFFECTS ON IL-17 PRODUCING T CELLS AND COLON EPITHELIAL BARRIER INTEGRITY

By

Neal Bhutiani

B.S., Duke University, 2009

M.D., Johns Hopkins University School of Medicine, 2013

A Dissertation Approved on

June 28, 2018

by the following Dissertation Committee:

Dissertation Director
Dr. Nejat K. Egilmez

Dr. Haribabu Bodduluri

Dr. Gerald W. Dryden

Dr. Bing Li

Dr. Robert C.G. Martin, II

Dr. Nathan W. Schmidt 


\section{ACKNOWLEDGEMENTS}

Dr. Qingsheng Li, an incomparably gifted scientist and exceptionally helpful person, without whom many of these studies would not have been successfully completed.

Mr. Charles D. Anderson for his constant assistance with everything from animal care to RNA extraction.

Drs. Magdia De Jesus and Heather Gallagher for graciously providing their expertise in immunofluorescence microscopy.

Dr. Stacia Furtado for providing us with therapeutic particles.

Drs. Venkatkrishna R. Jala and Rajbir Singh for their invaluable help with colon explant cultures.

Drs. Mostafa Fraig and William E. Grizzle for their timely and thorough pathologic review of our colon samples.

Dr. Lacey R. McNally for encouraging our use of multispectral optoacoustic tomography to noninvasively image colitis and track our nanoparticles, elegantly demonstrating the diagnostic power of this novel imaging modality for colorectal diseases.

My PhD Committee for their guidance and encouragement throughout my time as a graduate student.

Dr. Kelly M. McMasters for his support of my desire to pursue a second terminal degree during my surgical residency and establish myself as a surgeon-scientist as well as his friendship, mentorship, and honest advice.

Dr. Nejat K. Egilmez for his guidance, patience, and support during my time in his lab. I sincerely appreciate his ability to provide encouragement amidst frustration, his commitment to my long-term professional success as a surgeon-scientist, and his insistence on holding me to the highest of standards so that I might realize my potential.

My family, who has worked incredibly hard to provide me with every conceivable opportunity and to whom I owe every success. 


\begin{abstract}
COMBINATION ORAL IMMUNOTHERAPY WITH IL-10 AND IL-12 EFFECTIVELY TREATS COLON TUMORS VIA SYNERGISTIC EFFECTS ON IL-17 PRODUCING T CELLS AND COLON EPITHELIAL BARRIER INTEGRITY

Neal Bhutiani

June 28, 2018

In this dissertation, the relationship between colon cancer and inflammation, the utility of novel imaging modalities for diagnosis of colitis and cancer, and the therapeutic efficacy of orally delivered, particle-based immunotherapy for the treatment of colon cancer are evaluated.
\end{abstract}

In Chapters One and Two, multispectral optoacoustic tomography (MSOT) is demonstrated to effectively detect colon inflammation without the use of exogenous contrast prior to detection using conventional colonoscopy. Oral particle uptake is demonstrated in the distal small intestine and proximal colon, confirming site-specific delivery. 
In Chapter Three, administration of IL-10 and IL-12 containing particles is shown to act synergistically to significantly reduce tumor burden in the setting of established colon tumors. Cellular mechanisms deriving from effects on CD8+ T cells and T17 cells as well as a physiologic mechanism stemming from combination therapy's strengthening of colon epithelial barrier integrity are described.

In Chapter Four, the lack of efficacy of orally administered anti-PD1 therapy is demonstrated. IL-17 and $ү \delta$ T cells, but not CD4+ T cells, are shown to be critical mediators of treatment failure. The significant anti-tumor effect of combination treatment with either anti-IL-17A or anti- $y \delta$ TCR and anti-PD1 demonstrate exciting therapeutic targets for future clinical trials.

Finally, in Chapter Five, a clinically relevant model of colon cancer is described. This mutationally-driven model recapitulates the clinical scenario of single adenoma development, adenoma to carcinoma transition, carcinoma progression, and eventual metastasis to the liver. Such a model provides an excellent platform for preclinical evaluation of many different aspects of colon cancer. 


\section{TABLE OF CONTENTS}

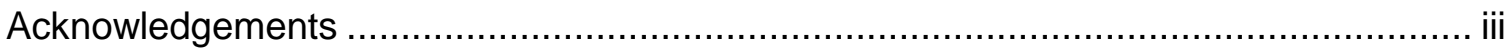

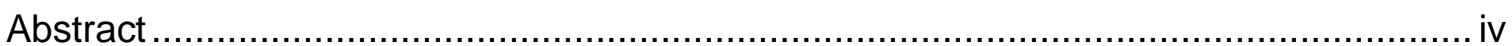

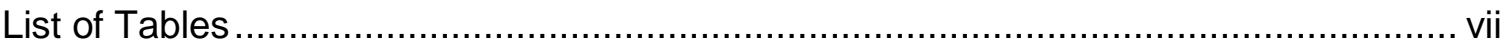

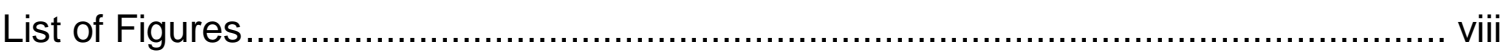

Introduction: Colon Cancer: Epidemiology, Molecular and Immunologic

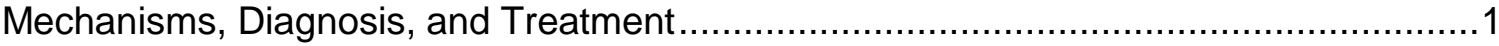

Chapter One: On the Relationship between Colitis and Cancer: Noninvasive

imaging of colitis induced by enterotoxic Bacteroides fragilis

Chapter Two: Tracking Oral Microspheres: Evaluating the uptake and distribution of orally-administered polylactic acid particles

Chapter Three: Treating Colon Cancer with Oral IL-10 and IL-12: The Synergy Mystery: Identifying the mechanisms behind the synergistic effect of IL-10 and IL-12 on colon tumor burden and delineating the effect of IL-10 and IL-12 on colon epithelial barrier integrity and its relationship to the efficacy of antineoplastic therapy.

Chapter Four: Saving Checkpoint Inhibitors: Elucidating mechanisms of failure of anti-PD1 therapy in colon cancer and identifying strategies to augment treatment efficacy

Chapter Five: The Next Frontier: Establishing a practical model for spontaneous colon tumors.

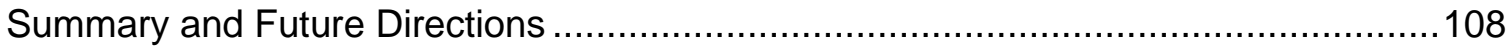

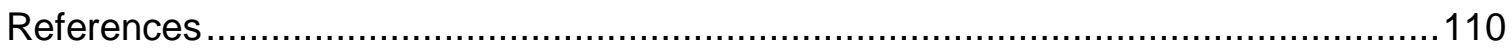

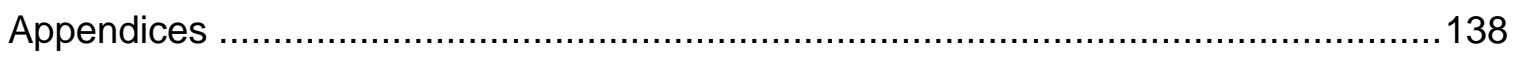

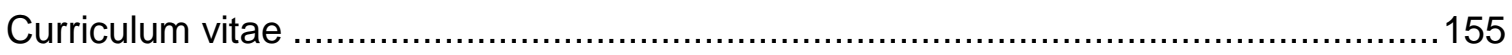




\section{LIST OF TABLES}

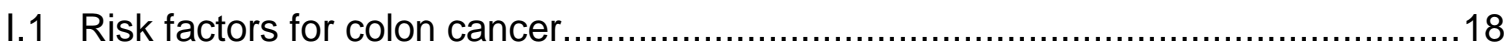

1.1 Murine endoscopic index of colitis severity for objective quantification of

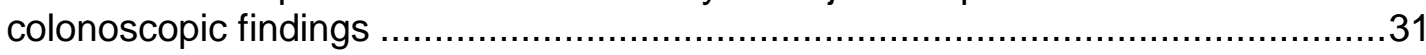




\section{LIST OF FIGURES}

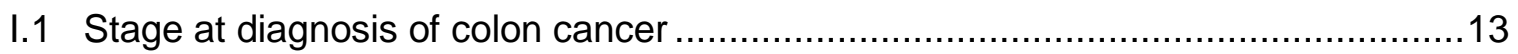

I.2 Survival distribution function of patients diagnosed with colon cancer by stage ......14

I.3 Sequence of genetic mutations in sporadic and colitis-associated colon cancer .....15

I.4 IL-17 signaling in normal versus neoplastic colon epithelial cells ..........................16

I.5 Physiologic alterations associated with disruption of colon epithelial barrier integrity

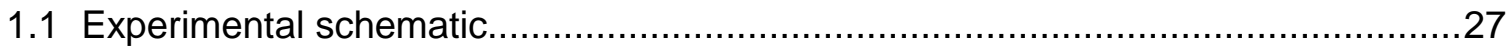

1.2 MSOT depicts inflammatory changes in murine colitis .........................................28

1.3 Mean MSOT signal intensity for oxyhemoglobin correlates with colitis severity score

1.4 Histology findings demonstrate inflammatory changes consistent with colitis .........30

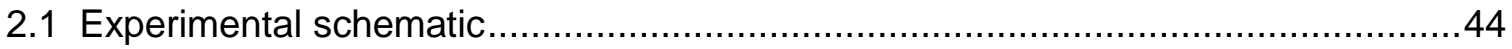

2.2 Characterization of the PLA-BSA-680 nanoparticles ..........................................45

2.3 Evaluation of optoacoustic signal within tissue phantoms of AF-680, PLA only, and PLA-BSA-AF680.

2.4 MSOT signal in gastrointestinal organs after oral gavage of either PLA-BSA-AF680 particles or BSA-AF680 alone .

2.5 Mean MSOT signal intensity correlates with particle localization...........................48

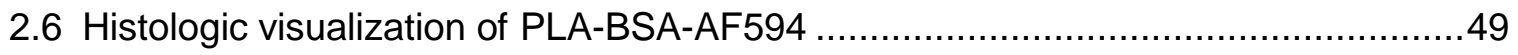

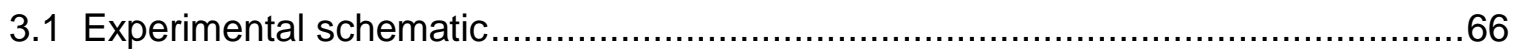

3.2 Oral delivery of cytokines to gut lamina propria..................................................67

3.3 Oral IL-10 reduces tumor burden in mice with established disease ........................68

3.4 Oral IL-10 and IL-12 act synergistically to eradicate established disease and 
improve overall survival

3.5 Distinct immunological effects of IL-10 and IL-12 on T-cell subsets are partially responsible for the antitumor synergy .....

3.6 Therapeutic synergy requires IFNy and is in part associated with the effects of cytokines on gut epithelial barrier integrity

3.7 IL-12 induces IFNy and IL-10RA expression in the colon ..................................

3.8 IFNy and IL-10 jointly promote tight junction protein expression in the colon ..........77

3.9 Hypothesized mechanism of action of IL-10 and IL-12 on colon epithelial barrier

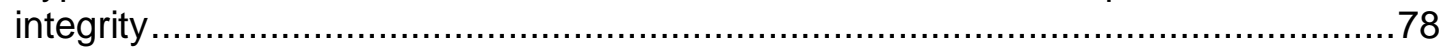

4.1 Oral anti-PD1 monotherapy is ineffective in treatment of established colon tumors

4.2 IL-17 blockade significantly improves the efficacy of oral anti-PD1 in treatment of established colon tumors

4.3 IL-17-based resistance to anti-PD1 therapy appears mediated by $ү \delta T C R+T$ cells but not CD4+ T cells

5.1 Mouse colonoscopy and adenovirus instillation

5.2 Gross and histologic demonstration of colon adenoma formation

5.3 Histologic evaluation of colon tumor, mesenteric lymph nodes, and liver 12 weeks after adenovirus instillation

5.4 Gross demonstration of colorectal liver metastases 48 weeks after adenovirus instillation 


\section{INTRODUCTION}

\section{COLON CANCER}

EPIDEMIOLOGY, MOLECULAR AND IMMUNOLOGIC MECHANISMS, DIAGNOSIS, AND TREATMENT

\section{Colon cancer epidemiology}

Approximately 4.5 percent of men and women in the United States will be diagnosed with colon cancer in their lifetime. ${ }^{1,2}$ In 2018 alone, nearly 100,000 people will be diagnosed with a new colon cancer. ${ }^{1}$ More significantly, in this same period, 50,630 people are expected die of colon cancer (including rectal cancer), making it the second leading cause of cancer-related deaths in the United States. ${ }^{1,2}$

While the disease has been decreasing in incidence over time as a result of improved screening leading to detection of and intervention upon pre-malignant lesions, it still represents a clinically significant disease process that impacts a significant number of people. Indeed, among those diagnosed with colon cancer, only about $40 \%$ are diagnosed with local disease (e.g. that confined only to the colon). ${ }^{2}$ The remaining $60 \%$ demonstrate evidence of disease in either the regional lymph nodes (35\%) or distant sites, particularly the liver (Figure 1). ${ }^{2}$ While 5-year survival approaches $90 \%$ for patients with localized disease with surgery and modern chemotherapy, that number 
drops to approximately $70 \%$ for patients with disease in their regional lymph nodes and less than $14 \%$ for patients with distant metastases (Figure 2$){ }^{2}$

Several risk factors have been shown to increase the risk of colon cancer (Table 1). ${ }^{1}$ Behavioral risk factors include physical inactivity, obesity, a diet high in processed and/or red meat, smoking, and moderate to heavy alcohol use. Aside from these, a family history of colon cancer has been associated with a two- to four-fold increased risk of developing colon cancer. ${ }^{3,4}$ This association becomes stronger with the larger the number of first degree relatives with colon cancer and the younger the age of those relatives at diagnosis.

Among those with a family history of colon cancer, approximately $5 \%$ have a genetic syndrome that increases their predisposition to developing the disease. ${ }^{5}$ The two most prominent, hereditary nonpolyposis colon cancer (HNPCC) and familial adenomatous polyposis (FAP), represent distinct clinicopathologic entities characterized by defects in DNA mismatch repair (MMR) machinery (MLH1, MSH2, MSH6, PMS2) and the adenomatous polyposis coli (APC) gene, respectively. ${ }^{1,6}$ Patients with HNPCC exhibit both a higher likelihood of developing colon cancer over the course of their lifetime (nearly $50 \%$ by age 70 ) as well as a younger median age at diagnosis (45 years). ${ }^{7}$ FAP patients, meanwhile, manifest innumerable polyps throughout their colon by preadolescence and have a nearly $100 \%$ risk of developing colon cancer by age $40 .^{8}$ 
In addition to lifestyle and genetic risk factors, medical comorbidities also significantly modulate an individual's risk for colon cancer. Type 2 diabetes and, more significantly, chronic inflammatory bowel disease (IBD; i.e. ulcerative colitis, Crohn's disease), have both been shown to increase an individual's risk of developing colon cancer. ${ }^{9-11}$ Indeed, patients with IBD have between a 2 and 5-fold greater likelihood of developing colon cancer over the course of their lifetime, particularly if diagnosed at a young age. This association derives from the persistent chronic inflammatory processes in the colon leading to increased cell turnover, higher mutation potential, and other elements which will be subsequently discussed in greater detail.

\section{Natural history}

In general, the pathogenesis of colon cancer adopts two distinct paradigms depending on whether cancer arises spontaneously or in the setting of IBD. ${ }^{12,13}$ In the former case, colon cancer arises as a result of an accumulation of spontaneous mutations that lead to hyperplasia, dysplasia, and eventually, development of invasive adenocarcinoma. Disruption of the epithelial barrier and microbial invasion results in "tumor-elicited inflammation," which enhances tumor growth. Meanwhile, in the setting of inflammatory bowel disease, chronic underlying colonic mucosal inflammation leads to mutations that result in adenocarcinoma formation, with tumor development resulting in propagation of the pro-inflammatory milieu.

The above differences are highlighted on a phenotypic and genotypic level. While sporadic colon cancer usually demonstrates a defined adenoma to carcinoma sequence with serial accumulation of genetic mutations in a relatively defined fashion, cancer that 
arises in the setting of inflammatory bowel disease usually is the result of progressively more dysplastic colon tissue with a different pattern and sequence of genetic mutations (Figure 3)..$^{12,14-17}$

Further complicating matters, sporadic colon cancer comprises several distinct subsets that behave differently on several levels. Microsatellite unstable tumors represent a particular entity that deserves attention. These cancers, which usually arise in the setting of silencing of the aforementioned mismatch repair gene MLH1, carry a favorable prognosis and demonstrate different levels of neoantigen exposure and immunogenicity than their microsatellite stable (MSS) counterparts. ${ }^{18-20}$ The distinction between these tumor types will be subsequently discussed in greater detail and represents a critical area of focus for novel colon cancer therapies.

\section{Screening for colon cancer}

As previously mentioned, screening efforts have played a major role in decreasing the incidence of colon cancer in the United States. Still, these measures, which can include colonoscopy, flexible sigmoidoscopy, or fecal occult blood test, demonstrate varying degrees of screening efficacy, with colonoscopy (the most invasive test) demonstrating the highest rate of polyp of dysplasia detection. ${ }^{1}$

The need for an invasive test for optimal colon cancer screening has posed several issues for both patients and the healthcare system. Many patients dislike the discomfort associated with colonoscopy, as it involves not only an invasive procedure but also a 
pre-test cathartic (bowel prep) for stool evacuation. ${ }^{21,22}$ Additionally, since the test requires the expertise of a skilled physician and nursing staff, the costs associated with screening are not insignificant, and patients must travel to centers with all necessary infrastructure to have their screening performed. ${ }^{1}$ For these and other reasons, screening rates have stagnated at approximately $65-70 \%$ in the United States.

The above factors, coupled with a larger number of American adults requiring colon cancer screening, point to the need for non-invasive, less operator-dependent modalities. Recently, CT colonography has emerged as a potential alternative to colonoscopy. ${ }^{23}$ However, it remains limited in its ability to detect polyps smaller than one centimeter. New technologies or new applications of existing technologies are thus needed as part of the effort to improve patient compliance with colon cancer screening guidelines and facilitate early detection of pre-malignant polyps, thereby reducing the overall burden of the disease.

\section{Current therapy for colon cancer}

While early stage colon cancer is treated effectively with surgical resection with or without systemic chemotherapy, locally advanced and metastatic disease portends a significantly worse prognosis. ${ }^{1,2}$ Indeed treatment of Stage 3 and Stage $4 a$ and/or $4 \mathrm{~b}$ of colorectal cancer $(\mathrm{CRC})$ involves multiple rounds of chemotherapy, surgery, and possibly hepatic embolization or ablation, conferring significant morbidity. 
Current standard of care for patients with locally advanced or metastatic colon cancer consists of 5-fluorouracil (5-FU) based chemotherapy (i.e. in combination with leucovorin and either oxaliplatin (FOLFOX) or irinotecan (FOLFIRI)). ${ }^{24-27}$ Systemic therapy in these patients focuses on delaying progression of disease and, if possible, reducing their disease burden/thus rendering patients candidates for resection. ${ }^{28,29}$ Additionally, chemotherapy is employed in a subset of patients who undergo resection thought to be at high risk for recurrence postoperatively. This usually consists of between 3 and 6 months of FOLFOX or an oral 5-FU analog (capecitabine) plus oxaliplatin. ${ }^{30}$ Patients with isolated lung or liver metastases may be candidates for metastatic tumor resection, which, in appropriately selected patients, can significantly improve survival. ${ }^{31-34}$

In patients receiving up-front chemotherapy for unresectable disease, approximately 30 $45 \%$ of patients respond to first-line therapy, and overall survival is approximately 20 months. ${ }^{26,35,36}$ Meanwhile, patients receiving postoperative chemotherapy exhibit approximately $75 \%$ disease free survival at 5 years. ${ }^{30}$ Still, these chemotherapy regimens can cause significant toxicity, particularly nausea and vomiting, neurotoxicity (in the case of oxaliplatin), hepatotoxicity, and neutropenia, in over $60 \%$ of patients. ${ }^{37}$ Indeed, the risk of severe toxicity increases among older patients, those with pretreatment comorbidities, and poor performance status.

In recent years, targeted therapies against VEGF and EGFR have become standard elements of the antineoplastic armamentarium in patients with advanced colon cancer. Anti-VEGF and Anti-EGFR agents. The former group, which includes bevacizumab and aflibercept and targets angiogenesis and aim to decrease blood supply to the tumor, has 
been shown to be effective either as monotherapy or in conjunction with the multiagent chemotherapy regimens described above..$^{38-40}$ However, its use significantly increases bleeding risk among patients undergoing surgery and incidence of severe toxicity. ${ }^{41-43}$ The latter group, which blocks pro-tumorigenic signaling through the epidermal growth factor pathway receptor, has demonstrated effect in patients with wild type $R A S$ oncogenes either as monotherapy or in combination with irinotecan- or, to a lesser extent, oxaliplatin-based regimens. ${ }^{44,45}$ While these agents have a less severe side effect profile than anti-VEGF agents, they are associated with an increase in toxicity when added to multi-agent chemotherapy regimens. ${ }^{46}$ These statistics, together with those presented above, highlight the importance of developing additional therapies for treatment of advanced stage colon cancer.

The immune system in colon cancer

As with many cancers, the immune system has been shown to play a significant role in intrinsic prevention of colon cancer. In general, this involves both innate and adaptive immunity. Specifically, natural killer (NK) cells have been demonstrated to prevent tumors and control their growth through recognition of activating ligands and decreased major histocompatibility complex I expression on tumor cells. ${ }^{47,48}$ Indeed, increased NK cell infiltration of colon tumors has been linked with improved prognosis in patients with colon cancer. ${ }^{49,50}$ Additionally, a subset of tumor infiltrating macrophages (M1 or M1-like macrophages) not only directly target tumor cells, but also increase major histocompatibility complex (MHC) and costimulatory molecule expression in addition to producing pro-inflammatory cytokines like IL-12 and TNFa, thereby augmenting both the innate and adaptive immune responses. ${ }^{51}$ 
As previously alluded to, colon tumor cells, like many other kinds of cancer, overexpress certain antigens that allow for their recognition by the (adaptive) immune system. Many of these antigens represent normal self-antigens that are simply overexpressed in tumor tissue (e.g. carcinoembryonic antigen (CEA), MUC-1, and p56) or proteins that result from mutations in genes such as Kras and $p 53 .^{52,53}$ Prevalence of immunogenic tumor neoantigens is further increased in microsatellite instability (MSI) high tumors, leading to greater lymphocyte infiltration of the tumor. ${ }^{54,55}$ This phenomenon, is, to an extent, counterbalanced by increased regulatory $\mathrm{T}$ cell infiltration which can blunt the anti-tumor immune response. ${ }^{56}$ Still, the importance of tumor infiltration by cytotoxic CD8+ T cells and memory CD8+ T cells cannot be overstated. Patients with higher proportions of infiltrating effector and memory CD8+ T cells have been shown to have improved prognosis across stages. ${ }^{57-61}$ Moreover, assessing these two parameters outperformed conventional tumor-nodes-metastasis (TNM) staging in predicting recurrence in these patients, highlighting the importance of the anti-tumor immune response in colon cancer. $^{62}$

Conversely, regardless of the mechanism by which colon cancer arises, immune dysregulation plays a major role in survival of tumor cells, tumor evasion of anti-tumor immune responses, and downregulation of said immune responses. CD4+ CD25+ FoxP3+ T regulatory cells exert immunosuppressive effects that blunt anti-tumor responses through release of cytokines like TGF $\beta$ and metabolites like adenosine. ${ }^{56,63}$ Regulatory $\mathrm{T}$ cell accumulation in tumors appears largely mediated by transformation of CD4+ T cells via TGF $\beta$ signaling, which can be secreted by a number of cells, including 
immature DCs (e.g. those found under circumstances of inhibited DC maturation in the presence of VEGF). ${ }^{63-67}$

The importance of regulatory T cells to outcomes in colon cancer is highlighted by the fact that murine models of colon cancer have demonstrated that regulatory $T$ cell depletion was associated with improved response to anti-tumor vaccines. ${ }^{68}$ Similarly, in vitro depletion of regulatory $\mathrm{T}$ cells in peripheral blood of colorectal cancer patients augments lymphocyte responses against tumor-associated antigens. ${ }^{69,70}$

In addition to Tregs, myeloid derived suppressor cells exert similar immunosuppressive functions and inhibit the functions of CD8+ T cells. Their importance in promoting tumor survival and immunoevasion has been demonstrated in mouse models, where myeloid derived suppressor cell (MDSC) depletion resulted in improved anti-tumor response and tumor regression. ${ }^{71}$ These cellular mechanisms of immune evasion are complemented by the high expression of PD-L1 on the surface of colon tumor cells, which bind to PD-1 on T cells and induce anergy and apoptotic cell death. ${ }^{72,73}$

More so than in other cancer types, interleukin-17 has been shown to play a critical role in development and progression of colon tumors in both mouse models of disease and among patients with early stage colon cancer. ${ }^{61,74-76}$ Production of this cytokine by several different cell types (Th17 cells, y $\delta$ T cells, natural killer T cells, and innate lymphoid cells), appears induced by IL-23 generated by tumor associated myeloid cells due to weakened epithelial tight junction integrity and bacterial migration. ${ }^{77}$ Subsequent 
signaling through IL-17RA on tumor cells results in signaling cascades involving mitogen-activated protein (MAP) kinases, extracellular signal-regulated kinases (ERK), and NF-KB, among other factors, to promote survival and proliferation of neoplastic epithelial cells (Figure 4). ${ }^{77,78}$

Moving beyond the immune system itself, disruption of colon epithelial barrier integrity and bacterial translocation have also been implicated in colon tumorigenesis and progression. Regardless of the etiology of barrier disruption, the process promotes cell turnover and generation of a host of antimicrobial peptides and pro-inflammatory cytokines. ${ }^{77,79}$ The resulting change in the immune milieu essentially establishes a persistent pro-inflammatory state that is exacerbated by bacterial migration and the dysplastic transformation of epithelial cells (Figure 5). ${ }^{79}$

\section{Immunotherapy for colon cancer}

In light of the significant role of immune dysregulation in its development and progression, the oncology community has invested significant effort in evaluating the potential role for immunotherapy in the treatment of colon cancer. In preclinical studies, vaccines, checkpoint inhibitors, and adoptive cell therapies have demonstrated significant anti-tumor effect. However, as will be subsequently discussed, such therapies have met with more limited success in clinical trials.

Beginning over 20 years ago, researchers began investigating the potential of immunebased therapies in the treatment of colon cancer. Until recently, much of these efforts 
focused on non-specific immune stimulation in conjunction with multi-agent chemotherapy and development of colon cancer vaccines. The former efforts have proven effective, particular the combination of granulocyte-monocyte colony stimulating factor (GM-CSF) with gemcitabine and FOLFOX. ${ }^{80-82}$ In a phase III clinical trial in patients with advanced colon cancer, this regimen was shown to result in a near doubling of response rate and a $50 \%$ increase in progression free survival compared to treatment with FOLFOX alone ${ }^{82}$ The efficacy of this strategy was marked by increases in memory $T$ cells and increases in circulating regulatory $T$ cells.

Vaccination efforts have focused on immunization with a wide variety of agents, including $\beta$-human chorionic gonadotropin, carcinoembryonic antigen, mutant RAS peptides, autologous tumor cells, and antigens loaded onto dendritic cells (DCs). Among these, the autologous tumor cell BCG vaccine (OncoVax) has demonstrated the greatest success. $^{83,84}$ Studies demonstrated that OncoVax treatment resulted in decreased recurrence rates and improved 5-year recurrence free survival rates among patients with stage II colon cancer. A multicenter phase III study is currently underway for patients with stage II colon cancer comparing OncoVax plus surgery to surgery alone.

More recently, clinical trials investigating the effect of therapy with immune checkpoint inhibitors, particularly anti-PD1, have demonstrated a markedly different effect in patients with colon cancer based on their microsatellite status. Indeed, patients with MSI high tumors exhibit response rates of $40-50 \%$ with significant improvements in progression free and overall survival. On the contrary, patients with microsatellite stable 
tumors respond only rarely ( $<5 \%$ of cases). ${ }^{85-88}$ The mechanisms of anti-PD1 resistance in MSS colon tumors remain unclear, though recently published work suggests that TGF $\beta$ signaling as well as mutations in JAK1/2 may play a role in this process. ${ }^{89,90}$ Further preclinical studies aimed at elucidating such mechanisms and clinical trials attempting to use combination regimens to overcome this resistance are currently underway. 
Figures and Tables

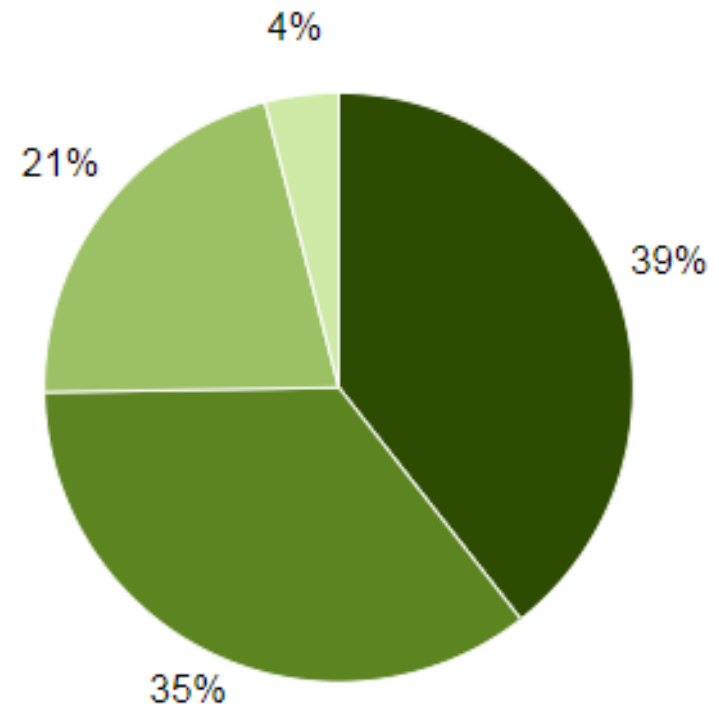

- Localized (39\%)

Confined to Primary Site

Regional (35\%)

Spread to Regional Lymph

Nodes

Distant (21\%)

Cancer Has Metastasized

Unknown (4\%)

Unstaged

Figure 1: Stage at diagnosis of colon cancer. Surveillance, Epidemiology, and End Results Program. Cancer Stat Facts: Colorectal Cancer.

https://seer.cancer.gov/statfacts/html/colorect.html. Accessed 5/9/2018, 2018. 


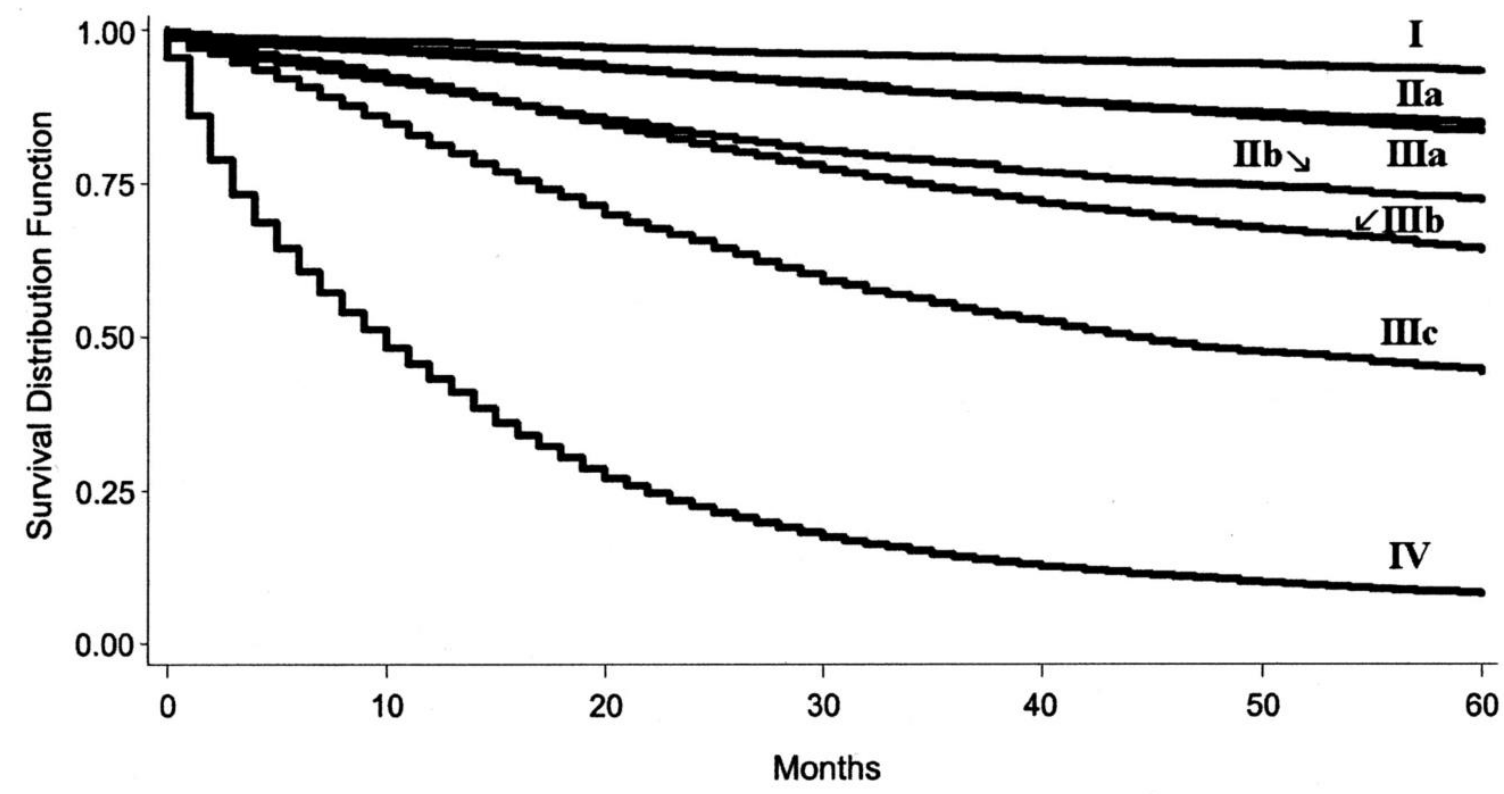

\begin{tabular}{|c|cc|ccc|ccc|}
\hline \multirow{2}{*}{ Stage } & \multicolumn{2}{|c|}{ 0 mo } & \multicolumn{3}{c|}{ 30 mo } & \multicolumn{3}{c|}{ 60 mo } \\
\cline { 2 - 9 } & $\begin{array}{c}\text { Survival, } \\
\text { \% }\end{array}$ & No. & $\begin{array}{c}\text { Survival, } \\
\text { \% }\end{array}$ & No. & $\boldsymbol{P}$ & $\begin{array}{c}\text { Survival, } \\
\%\end{array}$ & No. & $\boldsymbol{P}$ \\
\hline I & 100 & 14500 & 96.1 & 8,581 & - & 93.2 & 4514 & - \\
IIa & 100 & 28535 & 91.0 & 2,105 & $<.001$ & 84.7 & 8494 & $<.001$ \\
IIb & 100 & 5826 & 80.2 & 3,060 & $<.001 *$ & 72.2 & 1611 & $<.001^{*}$ \\
IIIa & 100 & 1989 & 91.4 & 1,120 & NS $\dagger$ & 83.4 & 551 & NS $\dagger$ \\
IIIb & 100 & 15946 & 77.3 & 7,786 & $<.001 \ddagger$ & 64.1 & 3579 & $<.001 \ddagger$ \\
IIIc & 100 & 8600 & 59.1 & 3,039 & $<.001$ & 44.3 & 1250 & $<.001$ \\
IV & 100 & 20802 & 17.3 & 1,832 & $<.001$ & 8.1 & 432 & $<.001$ \\
\hline
\end{tabular}

Figure 2: Survival distribution function of patients diagnosed with colon cancer by stage From: Colon Cancer Survival Rates With the New American Joint Committee on Cancer Sixth Edition Staging

J Natl Cancer Inst. 2004;96(19):1420-1425. doi:10.1093/jnci/djh275

J Natl Cancer Inst | (C) Oxford University Press 


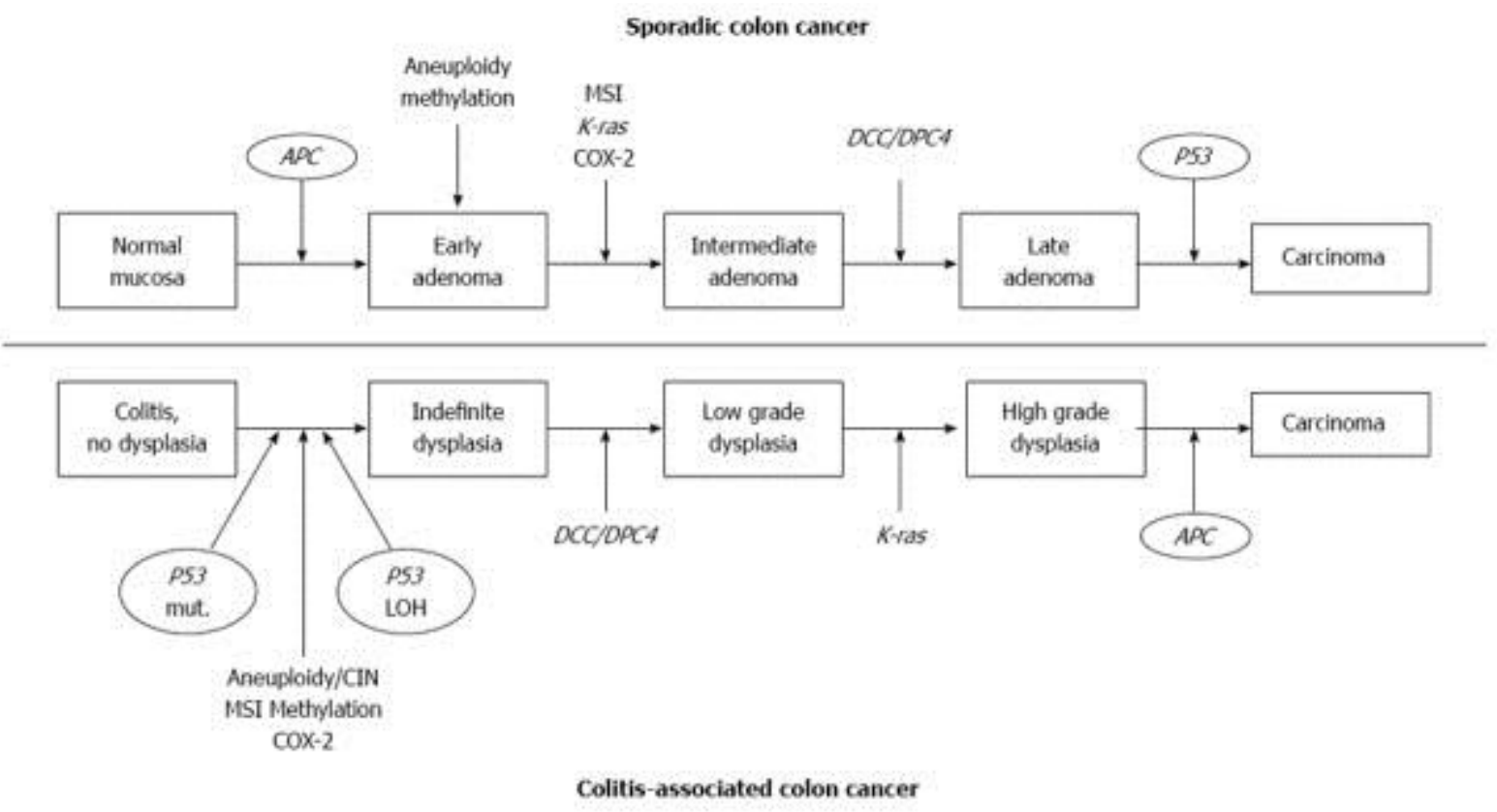

Figure 3: Sequence of genetic mutations in sporadic and colitis-associated colon cancer. Kim ER, Chang DK. Colorectal cancer in inflammatory bowel disease: the risk, pathogenesis, prevention and diagnosis. World journal of gastroenterology. 2014;20(29):9872-9881. 


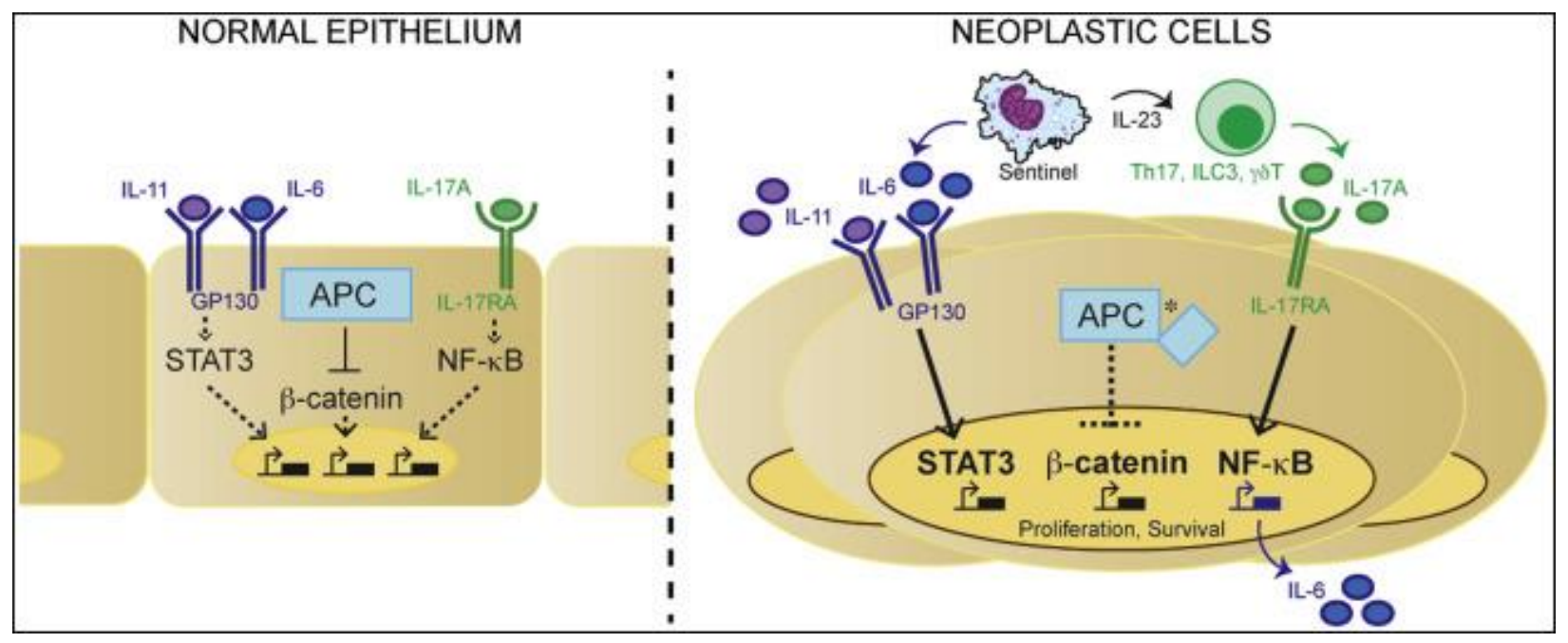

Figure 4: IL-17 Signaling in normal versus neoplastic colon epithelial cells. From Grivennikov SI, Wang K, Mucida D, et al. Adenoma-linked barrier defects and microbial products drive IL-23/L-17-mediated tumour growth. Nature. 2012;491(7423):254-258. 


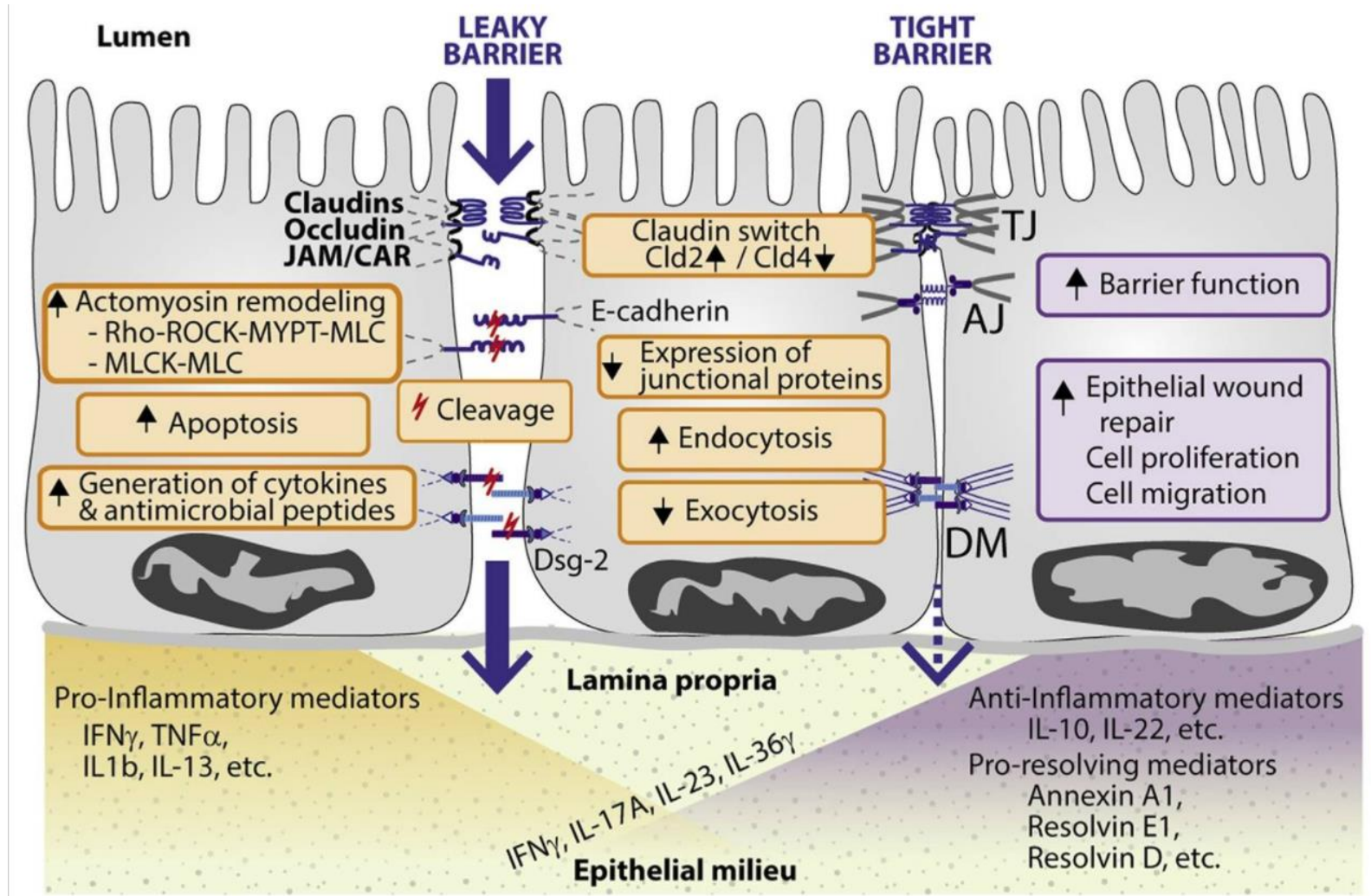

Figure 5: Physiologic alterations associated with disruption of colon epithelial barrier integrity. From Luissint AC, Parkos CA, Nusrat A. Inflammation and the Intestinal Barrier: Leukocyte-Epithelial Cell Interactions, Cell Junction Remodeling, and Mucosal Repair. Gastroenterology. 2016;151(4):616-632 
Relative

risk* $^{*}$

\section{Factors that increase risk:}

Heredity and medical history

$\begin{array}{ll}\text { Family history } & \\ 1 \text { first-degree relative } & \\ \text { More than } 1 \text { relative } & \\ \text { M7 } & 4.0 \\ \text { Relative with diagnosis before age } 45^{50} & 3.9 \\ \text { Inflammatory bowel disease }^{01} & 1.7 \\ \text { Diabetes }^{07} & 1.3\end{array}$

\section{Behavioral factors}

Alcohol consumption (daily average) ${ }^{145}$

2-3 drinks $\quad 1.2$

$>3$ drinks $\quad 1.4$

Obesity (body mass index $\left.\geq 30 \mathrm{~kg} / \mathrm{m}^{2}\right)^{104}$

Red meat consumption $(100 \mathrm{~g} / \text { day })^{131}$

Processed meat consumption $(50 \mathrm{~g} / \text { day })^{131}$

Smoking (ever vs. never) ${ }^{136}$

\section{Factors that decrease risk:}

Physical activity (colon) ${ }^{97} \quad 0.7$

Dairy consumption $(400 \mathrm{~g} / \text { day })^{119} \quad 0.8$

Milk consumption $(200 \mathrm{~g} / \text { day })^{119} \quad 0.9$

* Relative risk compares the risk of disease among people with a particular "exposure" to the risk among people without that exposure. Relative risk for dietary factors compares the highest with the lowest consumption. If the relative risk is more than 1.0 , then risk is higher among exposed than unexposed persons. Relative risks less than 1.0 indicate a protective effect.

(c)2017 American Cancer Society, Inc., Surveillance Research

Table 1: Risk factors for colon cancer. Colorectal Cancer Facts and Figures 2017-2019. Atlanta, GA: American Cancer Society;2017 
CHAPTER ONE

ON THE RELATIONSHIP BETWEEN COLITIS AND CANCER

NONINVASIVE IMAGING OF COLITIS INDUCED BY ENTEROTOXIC BACTEROIDES

FRAGILIS

\section{Introduction}

Inflammatory bowel disease (IBD) has grown increasingly prevalent over the last half century, with an annual incidence of approximately 250-300 per 100,000 persons in North America. ${ }^{1}$ Diagnosis usually involves a combination of factors identified in a patient's history and on physical examination, stool studies, and ultimately, colonoscopy with multiple biopsies. ${ }^{2}$ Furthermore, given the increased risk of colorectal cancer in patients with IBD, current practice guidelines recommend annual screening colonoscopies 8-10 y after onset of disease to assess for dysplastic changes and status of disease. ${ }^{3}$ In addition to being especially uncomfortable for patients with IBD, colonoscopy represents an invasive procedure that carries risk from both sedation or anesthesia and colonic perforation. To date, no noninvasive imaging modalities have 
been developed that accurately identify early inflammatory changes in patients' small bowel and colon, that can be used longitudinally to monitor the status of disease, or that detect changes concerning for malignancy.

Multispectral optoacoustic tomography (MSOT) represents a novel modality that detects sound waves resulting from specific molecular excitation by light. Specifically, laser stimulation of tissues results in generation and emission of ultrasound waves. Ultrasound waves demonstrate a significantly decreased scatter compared with light waves before reaching the detecting transducer. ${ }^{4}$ Thus, MSOT retains speed and sensitivity while having an improved signal-to-noise ratio compared with conventional radiographic imaging modalities (e.g., CT and $\mathrm{MRI}^{4-13}$ ). To date, MSOT has demonstrated a resolution at $78.9 \mathrm{~mm} .^{5,7}$ Furthermore, it can detect changes in tissue architecture and the presence of oxygenated and deoxygenated hemoglobin, ${ }^{5-10}$ permitting evaluation of changes in structure and vascularity, common in IBD. Recently, MSOT instrumentation has evolved and includes the potential for combination of detecting both optoacoustic and ultrasound signals, which increases its radiologic capabilities. Currently, MSOT has been used to effectively image tumor xenografts ${ }^{5-9}$ as well as several orthotopic tumor models, including pancreatic adenocarcinoma, ${ }^{6,7}$ but not to specifically assess inflammatory or dysplastic changes in the bowel in murine models.

Colonization with enterotoxic Bacteroides fragilis (ETBF), a pathogenic variant of a human intestinal commensal organism, has been implicated in the pathogenesis of IBD. ${ }^{14,15}$ Indeed, wild-type C57B/6 mice inoculated with ETBF after antibiotic-mediated depletion of intestinal flora can either initiate colitis or worsen susceptibility to colitis induced by other means. ${ }^{14,15}$ Like colitis observed in IBD patients, the inflammation persists over time and comprises both local and systemic components. Here, we use 
this model of ETBF-induced colitis in C57B/6 mice to evaluate the ability of MSOT to detect intestinal and colonic inflammation.

\section{Methods}

\section{Mice}

C57B/6 mice (Jackson Labs) were bred in accordance with University of Louisville (UofL) Institutional Animal Care and Use Committee (IACUC) guidelines. Six- to 7-wkold mice of both genders were used for all experiments. A total of 9 mice were used for experiments. All experiments were conducted in accordance with UofL IACUC guidelines. Figure 1 shows an experimental schematic.

\section{Bacteria}

ETBF strain 86-5443-2-2 was cultured under anaerobic conditions on brain-heart infusion 1 clindamycin agar plates. Plates were streaked with bacterial stock stored in glycerol and subsequently incubated under anaerobic conditions at $37 \mathrm{C}$. After $48 \mathrm{~h}$, bacteria were harvested and suspended in liquid brain-heart infusion 1 clindamycin broth before being incubated under anaerobic conditions for another $24 \mathrm{~h}$.

\section{Induction of Colitis}

To deplete enteric pathogen load and facilitate ETBF colonization, mice were administered clindamycin $(0.1 \mathrm{~g} / \mathrm{L})$ and streptomycin $(5 \mathrm{~g} / \mathrm{L})$ dissolved in drinking water for $4 \mathrm{~d}$ before bacterial inoculation. Approximately $1 \times 10^{8}$ bacteria suspended in $200 \mathrm{~mL}$ of phosphate buffered saline were then administered via oral gavage into the gastrointestinal tract of these mice. Mice that received neither antibiotics nor bacteria were used as controls. All mice were fed casein-based, low-anthocyanin chow ad libitum (TekLad 2920X; Envigo) and monitored closely for signs of dehydration and provided daily with electrolyte-rich gel supplements. 
Imaging and statistical comparison

MSOT imaging was performed as previously described. ${ }^{5}$ Briefly, mice were anesthetized with $1.6 \%$ isoflurane inhalant delivered in $0.8 \mathrm{~L}$ of medical air and $0.1 \mathrm{~L}$ of $\mathrm{O} 2$, then depilated using a combination of shaving and application of Nair with aloe (Church \& Dwight Co.), which was removed with moist gauze. Mice were subsequently placed prone and imaged from the superior thorax to inferior pelvis with the MSOT system InVision 256TF (iThera Medical) using wavelengths of $680,710,730,740,760,770$, $780,800,850$, and $900 \mathrm{~nm}$ with 25 averages per wavelength and an acquisition time of $10 \mu \mathrm{s}$ per frame. Accurate subject positioning within the MSOT device was ensured using the device's image preview function. Mice were evaluated before bacterial inoculation (untreated normal control), $2 \mathrm{~d}$ after bacterial inoculation, and $7 \mathrm{~d}$ after bacteria inoculation. Images were reconstructed via backprojection with $75-\mu \mathrm{m}$ resolution. MSOT values for oxygenated and deoxygenated hemoglobin were determined using MSOT imaging software (ViewMSOT 3.5) and compared using linear regression (JMP software; JMP, SAS Institute Inc.). Region-of-interest analysis was used to determine oxy- and deoxy-hemoglobin separately (6) within the colon using a 3.5-mm2 ellipse on 4 regions of the mouse colon per time point. The mean oxygenated hemoglobin signal intensity among the 4 regions was determined and compared using a 1-way ANOVA test with Tukey honest significant difference with JMP software. Differences were considered significant for a $\mathrm{P}$ value of less than 0.05 .

\section{Colonoscopy and statistical comparison}

Mice were injected intraperitoneally with $0.1 \mathrm{~mL} / 20 \mathrm{~g}$ mouse weight of a ketaminexylazine cocktail ( $87.5 \mathrm{mg} / \mathrm{kg}$ ketamine $+12.5 \mathrm{mg} / \mathrm{kg}$ xylazine). After an adequate level of anesthesia was achieved, an 8-French pediatric cystoscope was introduced into the 
mouse anus and advanced proximally until the scope could no longer be advanced. ${ }^{15,16}$ The colonoscope was then withdrawn slowly. The Murine Endoscopic Index of Colitis Severity was used to quantify colitis severity (Table 1). Colitis severity score was compared among and between groups using 1-way ANOVA with Tukey honest significant difference. Correlation between mean MSOT oxygenated and deoxygenated hemoglobin intensity and colitis severity score for mice evaluated with both MSOT and colonoscopy at all 3 time points was assessed using Pearson correlation. Statistical analyses were performed using JMP software. Differences were considered significant for a $P$ value of less than 0.05 .

\section{Histology}

After colonoscopy, mice were euthanized. The colon was resected in its entirety and flushed twice with phosphate-buffered saline to evacuate residual stool. Colons were then bisected, fixed in $10 \%$ neutral-buffered formalin, and embedded in paraffin. Sections were cut at 6- $\mu \mathrm{m}$ thickness and stained using hematoxylin and eosin. Samples were masked to the pathologist on histologic analysis by a diagnostic pathologist at the University of Alabama, Birmingham, Department of Pathology.

\section{Results}

Mice treated with ETBF showed a mild increase in vascularity $2 \mathrm{~d}$ after bacterial inoculation (Figs. 2 and 3), with an increase in mean signal intensity of oxygenated hemoglobin compared with untreated mice (1.150 vs. 2.716 MSOT arbitrary units compared with untreated mice; $P=0.004)$. These findings were more prominent $7 \mathrm{~d}$ after inoculation, with increased mean signal intensity of oxygenated hemoglobin (1.150 vs. 2.716 vs. 3.422 MSOT arbitrary units for controls vs. $2 \mathrm{~d}$ after ETBF vs. $7 \mathrm{~d}$ after $\mathrm{ETBF}, \mathrm{P}=0.0002$ ) and the development of punctate lesions on the colonic surface (Fig. 
2C). Deoxygenated hemoglobin signal obtained via MSOT remained similar in all mice evaluated regardless of treatment or time point.

The findings on MSOT correlated well with colonoscopic findings $(r=0.82, P=0.013)$. Compared with untreated controls, mice at $2 \mathrm{~d}$ after ETBF inoculation demonstrated an increased colitis score (1.5 vs. 2.5 ), with mild colonic blunting and slightly deformable stool (Figs. 2D and 2E). At $7 \mathrm{~d}$ after ETBF inoculation, mice displayed colonic blunting, vascular aneurysms, fibrin coats, patchy granularity, and deeply deformable stool (Fig. $2 \mathrm{~F}$ ), yielding an average colitis score of 5.5 (Figs. 2 and 3B).

Additionally, findings on MSOT and colonoscopy corresponded well with masked histologic analysis. Mice showed evidence of increasing inflammatory cell infiltrate and architectural distortion at both 2 and $7 \mathrm{~d}$ after ETBF inoculation compared with controls (Fig. 4). A similar progression was also evident comparing mice 2 and $7 \mathrm{~d}$ after ETBF inoculation. Findings were consistent across all mice in each group.

\section{Discussion}

Our findings demonstrate that MSOT findings of hypervascularity and elevated levels of oxyhemoglobin are associated with inflammatory changes in the colon as well as inflammatory cell infiltrate evidenced on histology; this was associated with mild inflammatory changes on histology that were minimally detectable on colonoscopy in the same mice.

Clinically, MSOT offers multiple advantages over all current imaging and monitoring modalities including higher resolution without requiring exogenous contrast agents. Because quantification of oxygenated hemoglobin correlates with vascular changes 
seen on colonoscopy and inflammatory changes seen on histology, MSOT can provide an objective assessment tool that can be used to monitor the severity and progression. Additionally, given that polyposis and tumor growth are associated with local neo/hypervascularization, MSOT can be used to monitor IBD patients for tumorigenesis, with colonoscopy used only if concerning features are identified on imaging studies.

Use of MSOT for diagnosis and monitoring of IBD has the potential to significantly affect disease prognosis. The MSOT apparatus for use in human subjects consists of a handheld probe similar to bedside ultrasound. ${ }^{7,10,17,18}$ Improvements in depth of tissue penetration of optoacoustic imaging beyond $1 \mathrm{~cm}$ have been demonstrated in humans using the clinical MSOT system, Acuity, with up to a 5-cm depth achieved. ${ }^{17,18}$ Therefore, MSOT could be used to provide noninvasive screening for IBD in patients at high risk for developing the disease because of factors such as family history and ethnicity, thereby enabling earlier detection of disease and commencement of therapy to prevent progression. Additionally, small changes detected on MSOT that may predict clinical worsening of disease (e.g., increased vascularity, development of small polyps) can prompt alteration of medical therapy or more timely colonoscopy and, if necessary, surgery to prevent clinical worsening of disease or delayed detection of malignancy and risk of local invasion or metastasis. Conversely, intervals between invasive monitoring with colonoscopy can be increased in patients lacking significant changes in colonic and mesenteric vascularity and polyposis on MSOT over time. This would decrease monitoring cost as well as procedural risks and discomfort for IBD patients.

\section{Conclusions}

MSOT represents a noninvasive diagnostic modality that effectively identifies colitis in a murine model. Its diagnostic accuracy is at least equivalent to current standards of 
colonoscopy and tissue histology and surpasses that of conventional imaging modalities. With improvements in deep-tissue penetration, these factors, together with MSOT's detection of oxygenated and deoxygenated hemoglobin as endogenous contrast agents and correlation of signal intensity with colitis severity, would allow MSOT to serve as a viable modality for diagnosis and monitoring of patients with IBD for both progression of disease and development of neoplastic intestinal lesions. 


\section{Figures and Tables}

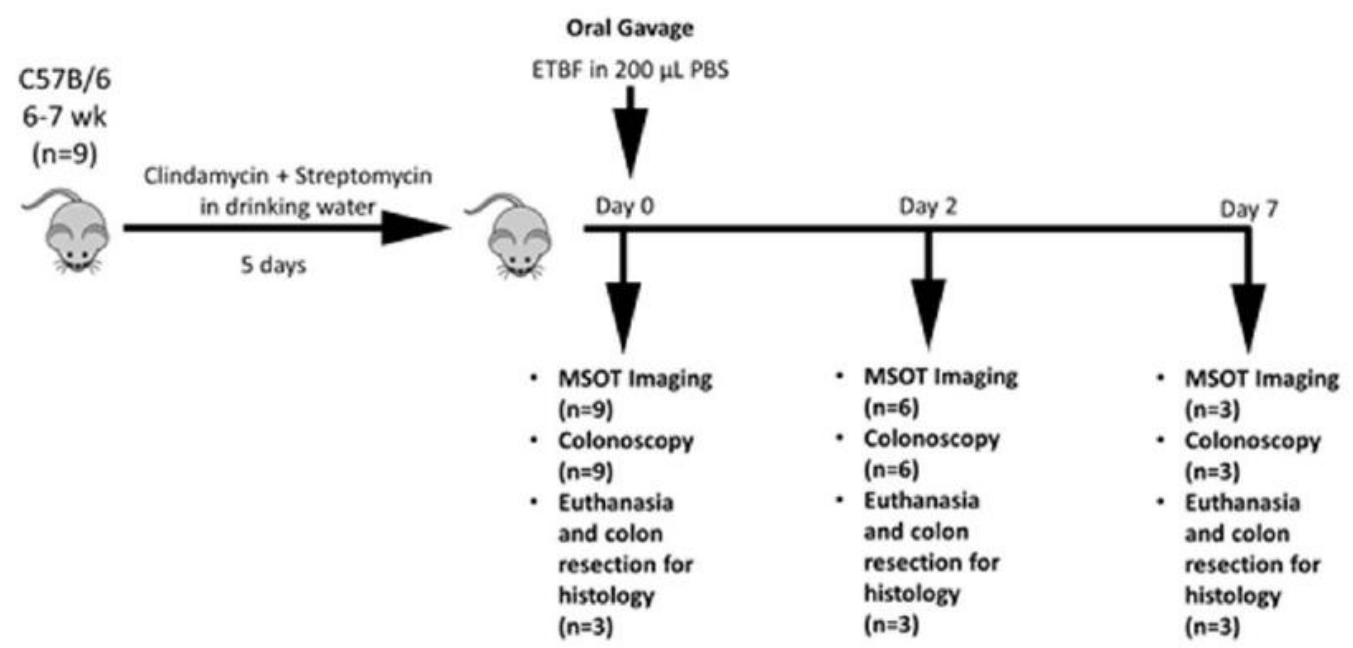

Figure 1: Experimental schematic 

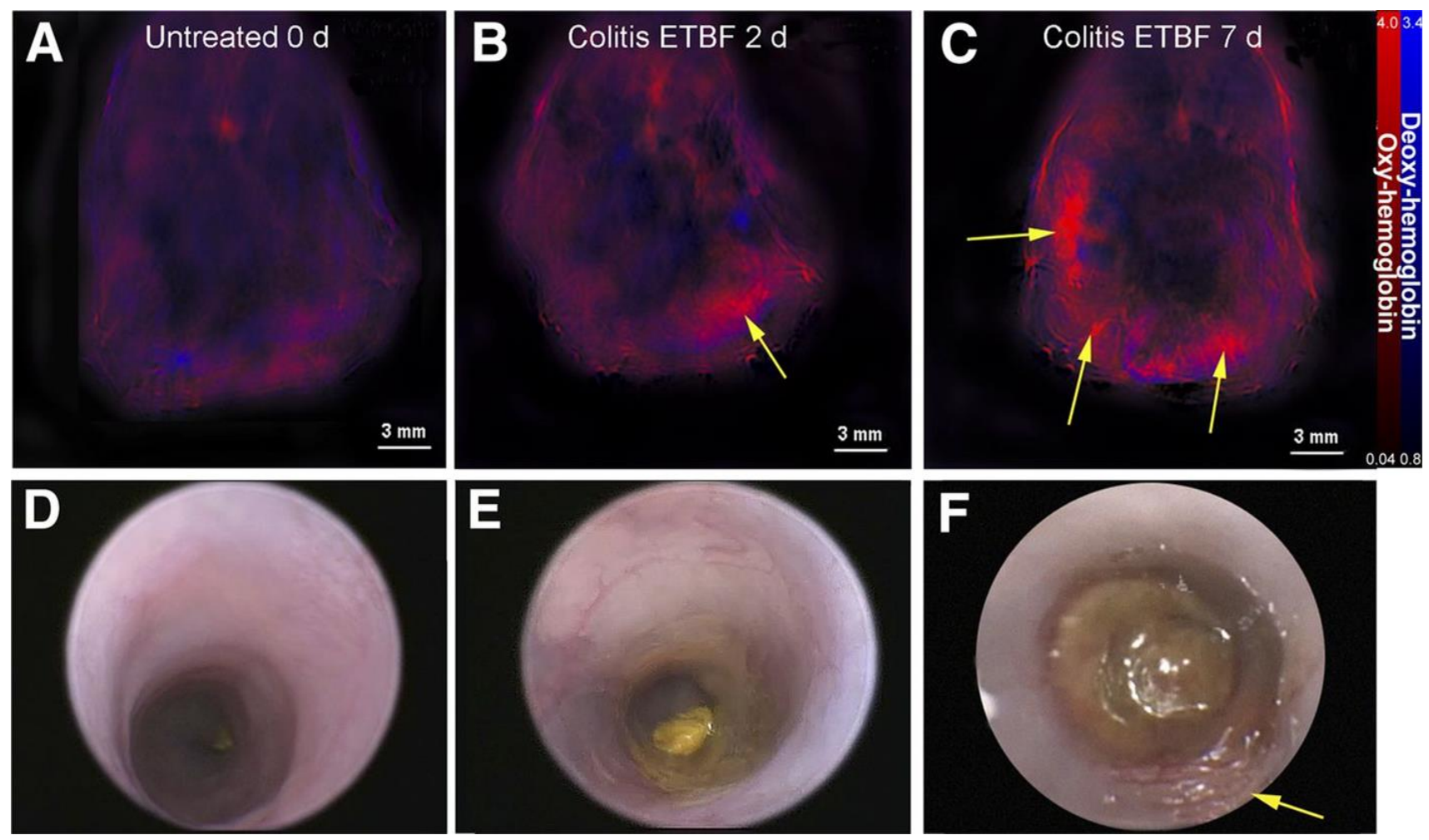

Figure 2: MSOT depicts inflammatory changes in murine colitis. Wild-type C57B/6 mice were orally inoculated with either phosphate-buffered saline alone (control) or ETBF. (A) MSOT imaging of mice before ETBF treatment (untreated). (B and C) Images of mice 2 and $7 \mathrm{~d}$, respectively, after bacterial inoculation. Arrows indicate concentrated areas of oxyhemoglobin corresponding to colitis (B and $\mathrm{C}$ ). Findings on MSOT were compared with colonoscopic findings (D-F) for each group of mice. Arrow indicates area of inflammation indicative of colitis $(F)$. 

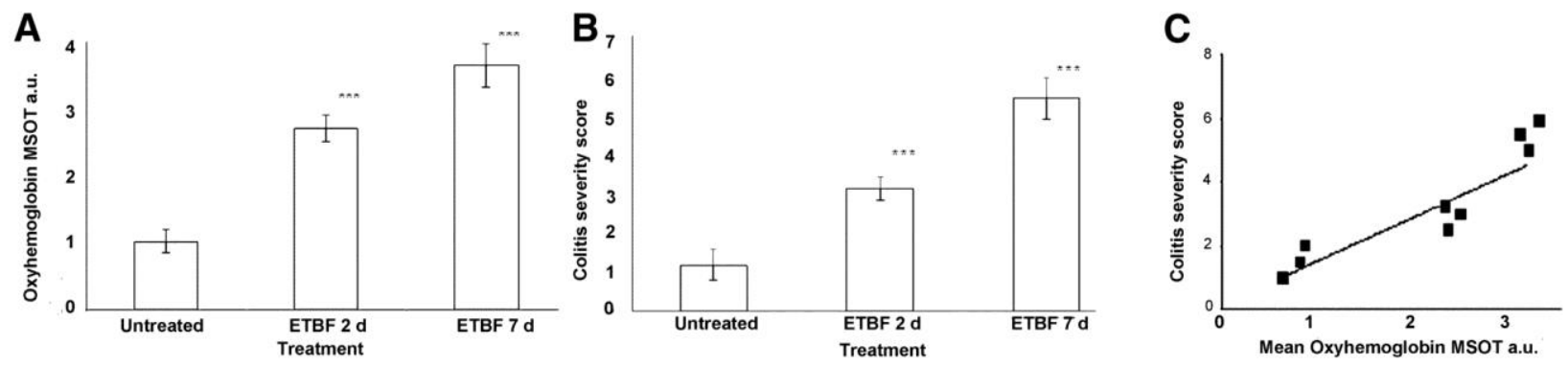

Figure 3: Mean MSOT signal intensity for oxyhemoglobin correlates with colitis severity score. Region of intensity measurements acquired for MSOT images were correlated to colitis severity score determined from colonoscopy images. (A) Mean signal intensity of oxyhemoglobin for each group (control, $2 \mathrm{~d}$ after ETBF inoculation, and $7 \mathrm{~d}$ after ETBF inoculation). ${ }^{* * *} \mathrm{P}<0.001$. (B) Average colitis severity score for each group. ${ }^{* * *} \mathrm{P}<$ 0.001. Error bars represent SD. (C) Correlation between mean signal intensity of oxyhemoglobin and mean colitis severity score for the 3 mice assessed using both MSOT and colonoscopy at all 3 time points $(r=0.82, P=0.013)$. a.u. $=$ arbitrary units. 


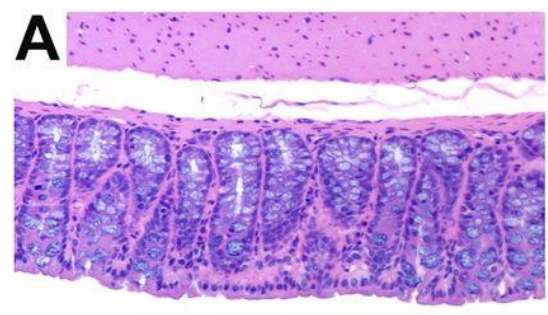

$50 \mu \mathrm{m}$

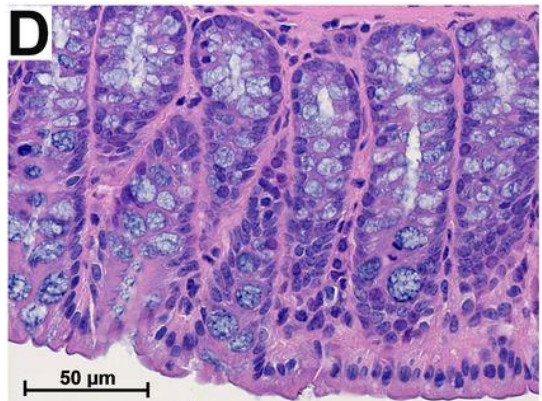

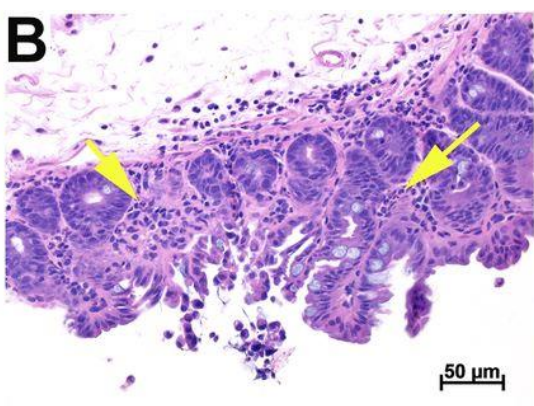
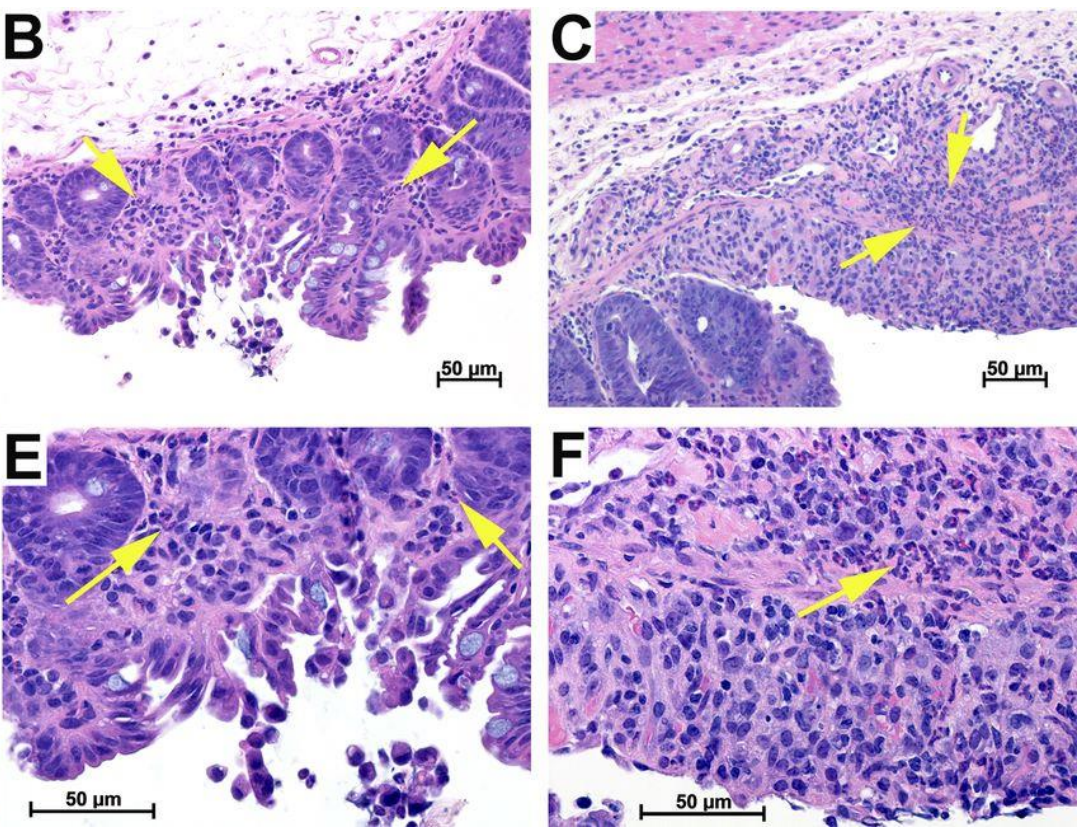

Figure 4: Histology findings demonstrate inflammatory changes consistent with colitis.

Hematoxylin and eosin analysis of mouse colon demonstrated no evidence of

inflammatory cell infiltrate in control mice (A and D) and progressively increasing

polymorphonuclear leukocyte infiltrate $2 \mathrm{~d}(\mathrm{~B}$ and $\mathrm{E})$ and $7 \mathrm{~d}(\mathrm{C}$ and $\mathrm{F})$ after ETBF

inoculation as indicated by arrows. Histology images shown in $\mathrm{C}$ and $\mathrm{F}$ are from mouse represented in Figure 1. 


\begin{tabular}{|c|c|c|c|c|c|}
\hline \multirow[b]{2}{*}{ Finding } & \multicolumn{5}{|c|}{ Murine endoscopic index of colitis severity } \\
\hline & 0 & 1 & 2 & 3 & Total \\
\hline Thickening of the colon & Transparent & Moderate & Marked & Nontransparent & $0-3$ \\
\hline Changes of the vascular pattern & Normal & Moderate & Marked & Bleeding & $0-3$ \\
\hline Fibrin visible & None & Little & Marked & Extreme & $0-3$ \\
\hline Granularity of the mucosal surface & None & Moderate & Marked & Extreme & $0-3$ \\
\hline \multirow[t]{2}{*}{ Stool consistency } & Normal + solid & Still shaped & Unshaped & Spread & $0-3$ \\
\hline & & & & & Overall, $0-15$ \\
\hline
\end{tabular}

Table 1: Murine endoscopic index of colitis severity for objective quantification of colonoscopic findings. Changes in colon thickness, vasculature, fibrin deposition, mucosal granularity, and stool consistence were all noted and assigned a score based on severity. These scores were summed to yield an overall colitis severity score. Table was modified from Becker, et al. (16). 
CHAPTER TWO

TRACKING ORAL MICROSPHERES

EVALUATING THE UPTAKE AND DISTRIBUTION OF ORALLY-ADMINISTERED POLYLACTIC ACID PARTICLES

\section{Introduction}

In both colorectal cancer and inflammatory bowel disease, effective, orally administered therapies represent the ideal route of drug delivery due to ease of administration and patient preference. Particularly in the case of immunomodulatory therapies, however, poor bioavailability and degradation by gastrointestinal enzymes prevents such administration from being more widely utilized. Additionally, noninvasively yet accurately imaging both gastrointestinal cancers and inflammatory bowel disease has proven difficult due to issues with resolution and reliance on grossly detectable differences in tissue density and vascularity. 
The development of micro- and nano-based carriers over the last twenty years represents an exciting mechanism to overcome these obstacles. Several studies have demonstrated the potential utility of orally administered therapeutic particles for treatment gastrointestinal (GI) diseases. ${ }^{1-5}$ While these molecules demonstrate mucoadhesive properties, which allow their binding to and uptake by the intestinal mucosa before subsequent trafficking to the mesenteric lymph nodes ${ }^{1}$, it is essential to track the biodistribution of these delivery vehicles within the gastrointestinal tract in a longitudinal manner to progress these treatments into clinical trials.

Multispectral optoacoustic tomography (MSOT) has recently emerged as a high resolution, non-invasive in vivo imaging modality. To date, optoacoustic imaging has largely identified solid tumors, namely melanoma, breast cancer, and pancreatic ductal adenocarcinoma, and various aspects of vascularity. ${ }^{6-12}$ As MSOT does not require exposure to radiation and nephrotoxic contrast agents, it represents a significant improvement in resolution compared to conventional imaging modalities (e.g. computed tomography $(\mathrm{CT})$, magnetic resonance imaging $(\mathrm{MRI})$, ultrasound $(\mathrm{U} / \mathrm{S})$ ) heightening its appeal as a tool for diagnosis and monitoring of various pathologies. However, unlike other solid organs, accurately imaging the gastrointestinal tract using MSOT has proven difficult owing to the motility of the small bowel and colon in conjunction with motion artifact introduced by murine respiration.

Our group has worked extensively with biodegradable polylactic acid particles for use in both intravenous and oral immunotherapy delivery. ${ }^{1-5,13-15}$ Furthermore, we have recently described high resolution imaging of the gastrointestinal tract using MSOT. ${ }^{16}$ 
This study aimed to evaluate the biodistribution and longitudinally track particle uptake through the murine Gl tract in vivo after oral administration.

\section{Methods}

\section{Orally Delivered Particles}

Polylactic acid particles containing either bovine serum albumin (BSA), BSA conjugated to AlexaFluor 680 dye, or BSA conjugated to AlexaFluor 594 dye were synthesized using a PIN as described previously. ${ }^{2}$ Briefly, BSA (Sigma Chemical Co., St. Louis, MO) or BSA-AF680 and PLA (Mr 24,000 and Mr 2,000; 1:1 (w/w)], Birmingham Polymers, Inc, Birmingham, AL) were suspended in methylene chloride (Fisher Scientific, Hampton, $\mathrm{NH}$ ) before being quickly poured into petroleum ether (Fisher) in order to form particles. After formation, solvent was removed by particle filtration and lyophilization overnight. Polylactic acid particles containing BSA-AF680 were characterized using dynamic light scattering and transmission electron microscopy. Multiple batches of particles were constructed to compare the repeatability of particle construction. Additionally, optical density of particles was measured using a spectrophotometer prior to drawing them into the gavage needle as well as after the particles were expelled from the needle. As less than 0.0001 change in OD was observed, we determined that the particles easily passed through at 24 gauge oral gavage needle. The encapsulation efficiencies for the BSA, BSA-AF680, or BSA-AF594 were extrapolated from the measurements of total protein encapsulated into the particles.

\section{Signal Assessment Ex Vivo in Tissue Phantoms}

To assess the ability of MSOT to detect AF-680 containing particles (hereafter PLABSA-AF680), PLA-BSA-AF680 was added to tissue phantoms designed to simulate optical properties of murine tissue. The tissue phantom was constructed by following procedures: Fixed cylindrical phantoms of $2 \mathrm{~cm}$ diameter were prepared using a gel 
made from distilled water containing Agar (Sigma Aldrich, St. Louis, MO, USA) for jellification (1.3\% w/w) and an intralipid 20\% emulsion (Sigma Aldrich, St. Louis, MO, USA) for light diffusion ( $6 \% \mathrm{v} / \mathrm{v})$, resulting in a gel presenting a reduced scattering coefficient of $\mu^{\prime s} \approx 10 \mathrm{~cm}^{-1}$. Blank PLA particles, PLA-BSA-AF60, or BSA-AF680 samples were inserted into cylindrical inclusions approximately $3 \mathrm{~mm}$ diameter. The gel used to construct the tissue phantom was used to seal the samples in the appropriate well prior to MSOT imaging MSOT imaging of the phantoms was done at a single position located approximately in the middle of the phantom. Data acquisition was performed at wavelengths of 680,710 , $730,740,760,770,780,800,850,900 \mathrm{~nm}$, using 10 averages per wavelength resulting in $1 \mathrm{~s}$ acquisition time per wavelength. Signal was measured using a Region of Interest method from MSOT images. Data obtained in MSOT arbitrary units (a.u.) was statistically compared using ANOVA.

Mice and particle delivery

Balb/c mice were placed on a low anthacyanin, casein-based diet for $48-72$ hours prior to particle administration. They were then orally gavaged with and equal admixture of

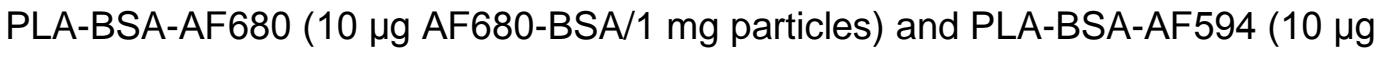
AF594-BSA/1 mg particles) suspended in phosphate buffered saline (PBS) or an equal admixture of naked AF680-BSA (hereafter BSA-AF680) and AF-594-BSA (hereafter BSA-AF594) suspended in PBS. This was done to allow for particle visualization using both MSOT and fluorescent microscopy. Mice each received a total of $5 \mathrm{mg}$ of PLABSA-AF680 + 5 mg PLA-BSA-AF594 in $100 \mu \mathrm{L}$ PBS or $50 \mu \mathrm{g}$ BSA-AF680 + 50 g BSAAF594 suspended in $100 \mu \mathrm{L}$ PBS. After hair removal using Nair with aloe (Church \& Dwight Co., Inc., New Jersey, USA), mice were imaged using multispectral optoacoustic tomography. 


\section{Multispectral Optoacoustic Tomography}

Multispectral optoacoustic tomographic (MSOT) imaging was performed as previously described. Briefly, after anesthesia with $1.6 \%$ isofluorane, mice were prepared for imaging using a combination of manual shaving and Nair cream with aloe (Church and Dwight Co., Princeton, NJ, USA $)^{17}$. Mice were subsequently imaged using the MSOT system InVision TF 256 (iThera Medical, Munich, Germany) using wavelengths of 680, $710,730,740,760,770,780,800,850$, and $900 \mathrm{~nm}$ with 25 averages per wavelength and an acquisition time of $10 \mu \mathrm{s}$ per frame. The water temperature was $35^{\circ} \mathrm{C}$ within the instrument during acquisition.

\section{MSOT Image Reconstruction and Analysis}

Raw data obtained with MSOT was reconstructed with multispectral analysis performed as previously described ${ }^{10,18}$. Spectral analysis was performed at wavelengths corresponding to deoxy-hemoglobin and BSA-680. Reconstruction was conducted using backprojection at a resolution of $75 \mu \mathrm{m}$ using ViewMSOT software version 3.5 (iThera Medical, Munich, Germany). The Multispectral Processing was conducted using Linear Regression with ViewMSOT 3.5, where known molar absorptivity spectra (e.g. for oxyhemoglobin, deoxy-hemoglobin, and nanoparticle) are used to model the relationship between chromophore concentration and MSOT signal over a range of wavelengths. The approach assumes knowledge about all absorbers present in the imaged tissue in order to correctly attribute contributions from the different wavelengths to the unmixed component images ${ }^{19,20}$. In order to ensure comparability among data sets, the reconstruction parameters (field of view, speed of sound, pixel size, and the high/low pass filters) and spectral unmixing parameters were consistently applied to all data. Spectral unmixing was performed in the absence of correction for fluence heterogeneities and attenuation as a function of tissue depth including spectral coloring. 
The location of organs was identified based upon vascular pattern. In addition, a region of interest (ROI) method was applied to determine signal strength in the stomach, liver, small bowel, colon, spleen, and mesenteric lymph nodes (MLN) of mice acquired at 1 min, $1 \mathrm{~h}, 6 \mathrm{~h}, 12 \mathrm{~h}, 24 \mathrm{~h}$, and $48 \mathrm{~h}$ post-gavage using ViewMSOT software and reported as MSOT a.u. The ROI was manually created with an ellipse drawing tool using the deoxy-hemoglobin spectrally unmixed component as a guide for organ location. The ROI area was kept constant for all image slices $3.5 \mathrm{~mm}^{2}$, thus creating a non-uniform elliptical prism volume of interest (VOI). The mean pixel intensity per cross-section in the VOI for the spectrally unmixed injected agent (BSA-680) was plotted as MSOT signal vs. position to assess the particle location. The maximal 'mean signal per cross-section' in the volume was used as a quantitative indicator of particle trafficking. Since optoacoustic signals using the detection geometry of this system are subject to out-ofplane contributions, this method was used to find the center of signal intensity and minimize variability from out-of-plane artifacts. The capacity of this optoacoustic system to deliver semi-quantitative data reflective of relative probe accumulation in vivo in murine models using the aforementioned reconstruction and multispectral unmixing methods was previously established. ${ }^{21}$ The MSOT a.u. values for the particle containing BSA-680 were compared using SAS 9.3 (Cary, NC, USA).

\section{Organ Histology and Microscopy}

After imaging, mice were euthanized and abdominal organs were embedded in paraffin, sectioned at 6 micron thickness, and stained with hematoxylin and eosin (H\&E) for evaluation using fluorescence microscopy. Images were acquired using both conventional white light and fluorescence lamps. MSOT images were correlated with fluorescence microscopy images to determine accuracy of MSOT based particle localization. For an experimental schematic, please see Figure 1. 


\section{Results}

PLA particles were characterized as to size, polydispersity index, and ability to encapsulate BSA-680. As is characteristic of the discrepancy between TEM and DLS particle sizing, PLA particles averaged $33 \mathrm{~nm}$ using TEM and $46 \mathrm{~nm}$ using DLS (Figures 2A-B). The generation of 3 batches of PLA-BSA-680 particles also resulted in similar sizing results along with similar polydispersity index of 0.11-0.14 (Figure 2C). Further comparison of the spectral signature of AF-680 and BSA-680 indicated very little spectral differences (Figure 2D). Assessment of particles in agar and intralipid tissue phantoms demonstrated that blank PLA particles produced no signal at any excitation wavelength (Figure 3). PLA-BSA-AF680 produced MSOT signal with intensity 32 MSOT a.u. This was slightly less intense than but otherwise similar to the signal produced by AF680 of 38 MSOT a.u. (Figure 3).

In in vivo studies, mice treated with PLA- BSA-AF680 exhibited MSOT signal in the stomach at 1 min after gavage. After gavage, signal was noted in the wall of the small bowel at starting at $1 \mathrm{hr}$ after gavage up through 24 hours after gavage, in the wall of the colon at 6, 12, and $24 \mathrm{~h}$, in the MLN 12 and $24 \mathrm{~h}$, and in the spleen 24 and 48h. No signal was detected in the liver. Maximum signal post-gavage was noted in the wall of the small bowel at $6 \mathrm{~h}$, colon at $24 \mathrm{~h}$, and MLN at $48 \mathrm{~h}$ (Figure 4). Figure 5 demonstrate the signal noted in each respective organ at 24 hours after gavage. Intralumenal and intraepithelial signal were differentiated through overlay of AF-680 signal on deoxyhemoglobin signal. Mean MSOT signal intensity in target organs at each timepoint corresponded with the visualized signal intensity (e.g. 24 hours, Figure 5). 
Compared to mice gavaged with PLA-BSA-AF680, those gavaged with BSA-AF680 demonstrated similar a much shorter duration of signal visibility on MSOT with very little signal detected after $1 \mathrm{~h}$, no signal detected after $6 \mathrm{~h}$, and decreased signal in target tissues. BSA-AF680 was detected in the stomach immediately after gavage (data not shown) and progressed through the lumen of the gastrointestinal tract over the course of $48 \mathrm{~h}$, with minimal signal noted in the wall of the small bowel and colon and no signal noted in the spleen or liver.

Particle localization on MSOT correlated well with findings on fluorescence microscopy, which demonstrated particle localization in organs identified on MSOT at each given timepoint (e.g. 24 hours, Figure 6). Specifically, particle uptake was visualized at the apical aspect of epithelial cells in the small bowel and colon and in the MLNs. BSAAF680 was visualized around splenic sinusoids at the timepoints when signal was observed on MSOT.

\section{Discussion}

Here, we describe high-resolution imaging of the murine gastrointestinal tract and histologically correlated, site-specific uptake of poly-lactic acid particles by cells in tissues along the gastrointestinal tract. To our knowledge, this represents the first description of such high resolution MSOT imaging of the murine gastrointestinal tract, specifically that which allows for tracking of orally delivered particles.

To date, high resolution gastrointestinal imaging using MSOT has proven challenging due to a combination of 1) intrinsic gastrointestinal motility, 2) lack of fixed organ position within the abdomen, 3) motion artifact conferred by respiration, and 4) artefactual signal produced by anthacyanins in murine food. Ensuring an adequate level of anesthesia, 
utilizing a casein-based, low-anthacyanin diet, and, most importantly, acquiring 25 averages per wavelength proved critical to enabling detection of organ specific particle localization in our studies. These techniques have also enabled us to image colitis in a murine model with detection of both general areas of inflammation/hypervascularity and individual blood vessels. ${ }^{16}$

In addition to the MSOT imaging protocol, the mucoadhesive properties of the PLA particles facilitated organ identification and histologic correlation with MSOT imaging. While much of the gavaged fluid-particle mixture passes through the gastrointestinal tract, mucoadhesion enables some of the particles to be retained and taken up by absorptive tissues. The mechanism of uptake remains unclear, but likely occurs via phagocytosis given the size of the particles ( $46 \mathrm{~nm}$ average diameter). The importance of mucosal adhesion is illustrated in comparing BSA-AF680 to PLA-BSA-AF680. The former passes through the Gl tract with minimal absorption, with the small amount of absorption that occurs likely mediated by pinocytosis or passive transport with water uptake. Meanwhile, the latter demonstrates discrete uptake and strong signal on both MSOT (Figure 4) and histology (Figure 6).

Indeed, this correlates well with our previous findings that MSOT can reliably detect individual nanoparticles as small as $75 \mathrm{~nm}$ in diameter. ${ }^{22}$ Together, the ability of MSOT to detect such small particles as well as its ability to detect fluorophores or other fluorescent contrast agents of various wavelengths present numerous opportunities for clinical imaging applications within the gastrointestinal tract. Of course, use of MSOT can avoid nephrotoxic contrast agents used in conventional imaging modalities such as CT and MRI. However, with the ability to detect multiple fluorophores, MSOT also can allow for particles to be administered orally and intravenously and thus enable 
simultaneous detection of inflammation, malignancy, and other pathologies.

Furthermore, varying particle size, protein or small molecule tags, and biochemical properties (e.g. porosity and pore size, chemical composition, etc.) can allow for tissue specific uptake based on organ or cell type (e.g. tumor cell). ${ }^{17,23}$

While MSOT accurately tracks particle uptake in the gastrointestinal tract and identifies fluorophore signal, it does not readily distinguish between encapsulated and naked fluorophore. For example, we observe signal of similar intensity in the colon at 24 hours and spleen at 48 hours, but the former is produced by encapsulated fluorophore and the latter by naked fluorophore. While this does not ostensibly impact imaging using particle-encapsulated fluorophore, it would become important in tracking fluorophoretagged particles. In the case of PLA particles in this study, it remains unclear at which point the particles are degraded. Discrete particles are observed in the small bowel, colon, and MLN but not in the spleen. Degradation could be a factor of particle time in circulation, a function of being in the lymphatic circulation, or both. Indeed, previous studies have demonstrated the lymphatic system's role in metabolizing elements of the intercellular matrix and connective tissues (e.g. hyaluronan). ${ }^{24}$ Particle-specific studies would be required to determine in vivo degradation kinetics, which would in turn impact potential clinical applications.

The findings of this study should be viewed in light of several limitations. All imaging was performed on Balb/c mice, as skin pigment results in signal artifact that prevents high-resolution imaging and would have prevented particle localization. Thus, the present of endogenous skin pigment (e.g. in the case of particularly dark-skin) would limit clinical MSOT utility using a hand-held probe, but would likely not inhibit an endoscopic probe in imaging such patients. Also, MSOT cannot detect non-light 
absorbing particles, so any clinical application would require incorporation of some absorbing material (e.g. protein conjugated fluorophore, gold) within the nanoparticle. All tissue was fixed in formalin for histology prior to H\&E staining. The totality of the processing may have resulted in the loss of some particles in target tissues at each timepoint. Finally, the results presented herein do not necessarily reflect particle content delivery or reflect information regarding the minimum dose of particles that results in detectable MSOT signal in each target organ, but rather, describe the ability to monitor particle travel in vivo using MSOT,

Despite these limitations, our findings present a number of opportunities for future investigation. With the recently-developed clinical MSOT apparatus, nanoparticletracking studies in the gastrointestinal tract can be replicated in humans to assess the diagnostic utility of MSOT in the clinical setting. Within the last several months, researchers at the University of Erlangen-Nurnberg Medical School demonstrated the ability of MSOT to detect inflammatory changes without exogenous contrast in human subjects. ${ }^{25}$ Evaluating MSOT's ability to track fluorophore-containing nanoparticles represents a logical extension of this work. In examining various iterations of particles and dyes, we would be able to correlate particle uptake with enterocolonic inflammation as well evaluate tumor-specific uptake in the setting of enterocolonic malignancy. Finally, using fluorophore-tagged nanoparticles, we could use MSOT to actively track theranostic nanoparticles to actively establish pharmacokinetics of contrast and/or drug delivery.

\section{Conclusions}

MSOT detects orally administered AF-680 dye encapsulated PLA particles in vivo. These particles demonstrate site-specific uptake in the wall of the small bowel, colon, 
and mesenteric lymph nodes with naked dye being visible in the spleen. MSOT tracking of fluorophore containing particles could improve monitoring of drug delivery and lead to more optimal individualized dosing schedules. Furthermore, with improved specificity, these particles could be further tested in humans in combination with the handheld MSOT to help surgeons identify sites of active disease or malignancy in the operating room. 


\section{Figures}

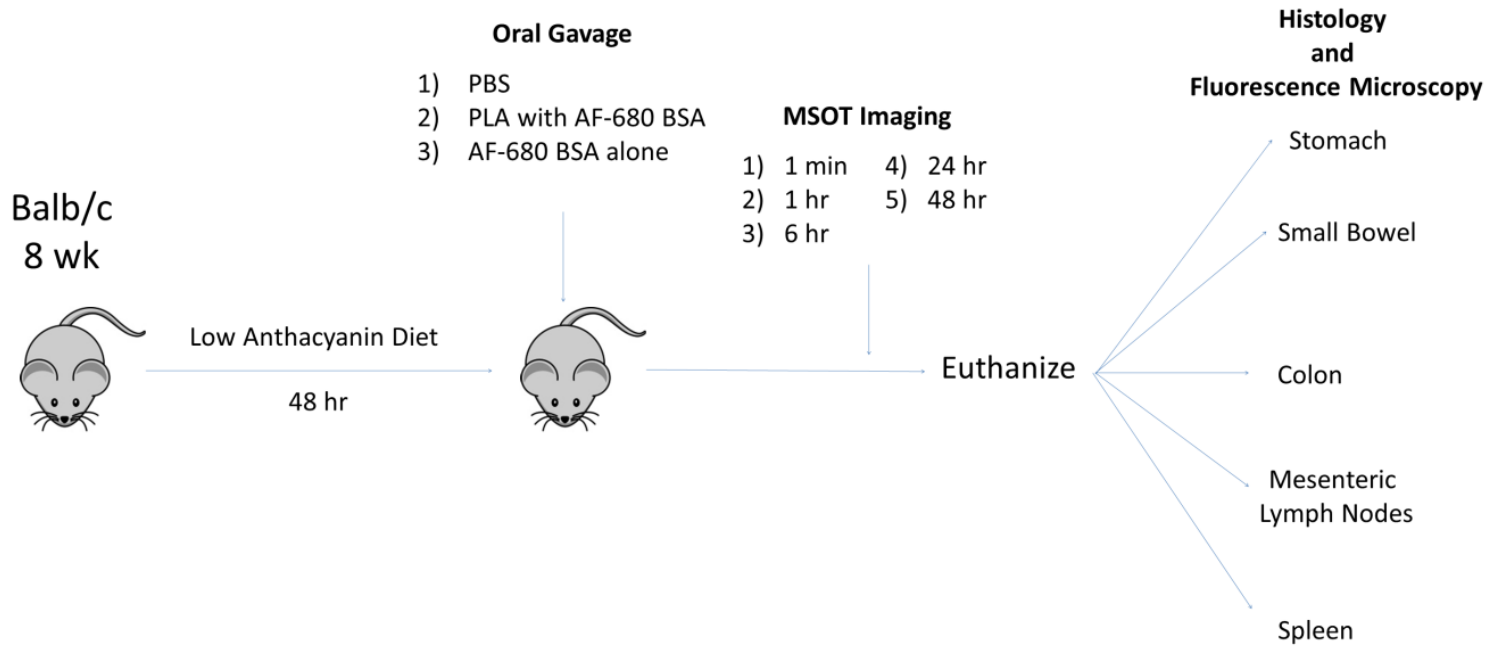

Figure 1: Experimental schematic for mouse experiments 

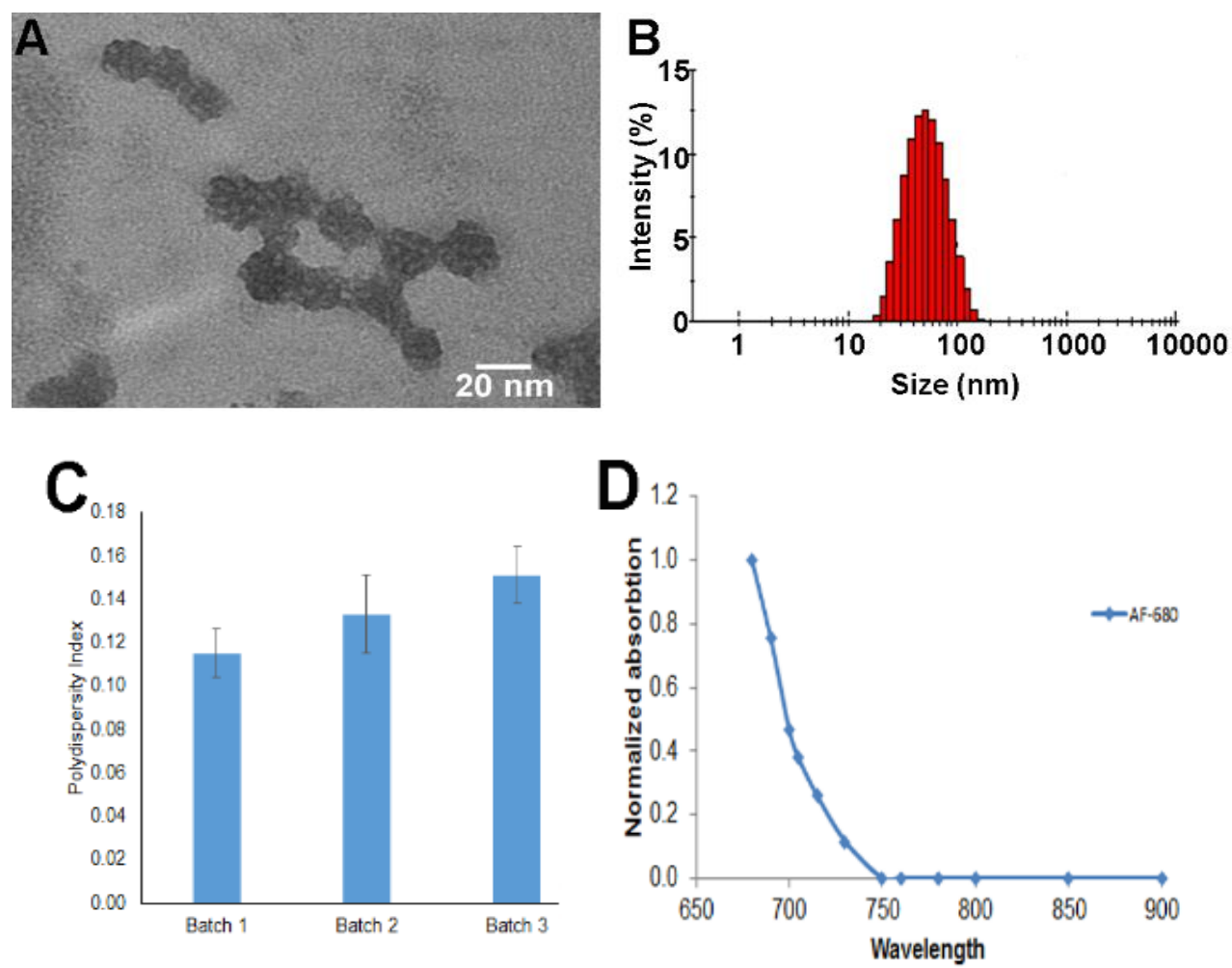

Figure 2: Characterization of the PLA-BSA-680 nanoparticles. A) Transmission electron micrograph (TEM) images with an average particle size of $33 \pm 12 \mathrm{~nm}$. B) Dynamic light scattering (DLS) of the PLA particles resulted in an average size of $44 \mathrm{~nm}$. C) Polydispersity Index (PDI) shows narrow polydispersed distribution as the PDI ranged from 0.12-0.135 over the course of 3 batches of particles evaluated. D) Spectral absorption was determined of AF-680 and BSA-680 which demonstrated a high degree of similarity. 


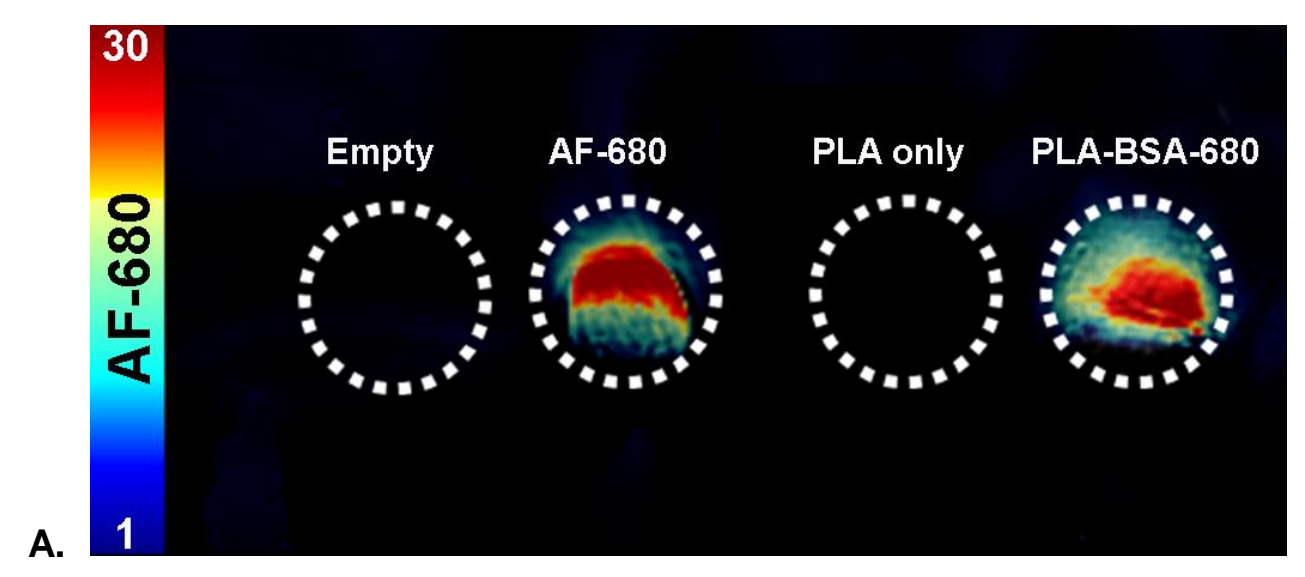

B.

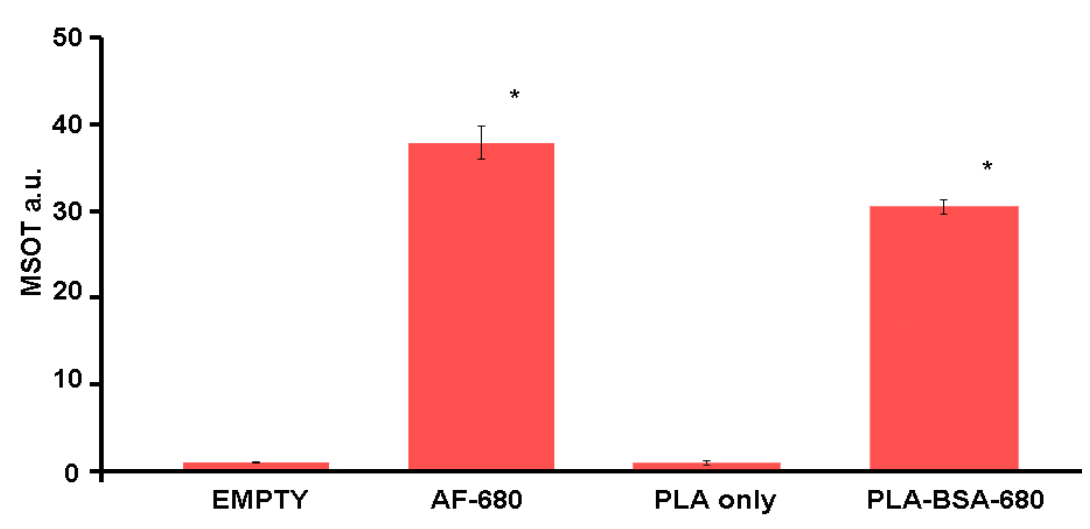

Figure 3: Evaluation of optoacoustic signal within tissue phantoms of AF-680, PLA only, and PLA-BSA-AF680. (A) MSOT signal of AF-680, particles only, and particleencapsulated AF-680 was determined using the spectral signature in Figure 2D in tissue phantoms clearly differs from particles alone. (B) Uptake of BSA-680 within the PLA particles was demonstrated by similarities of signal intensity between AF-680 and PLABSA-680 within tissue phantoms. Both AF-680 and PLA-BSA-680 had significantly higher signal $(p<0.05)$ than PLA only or empty. 


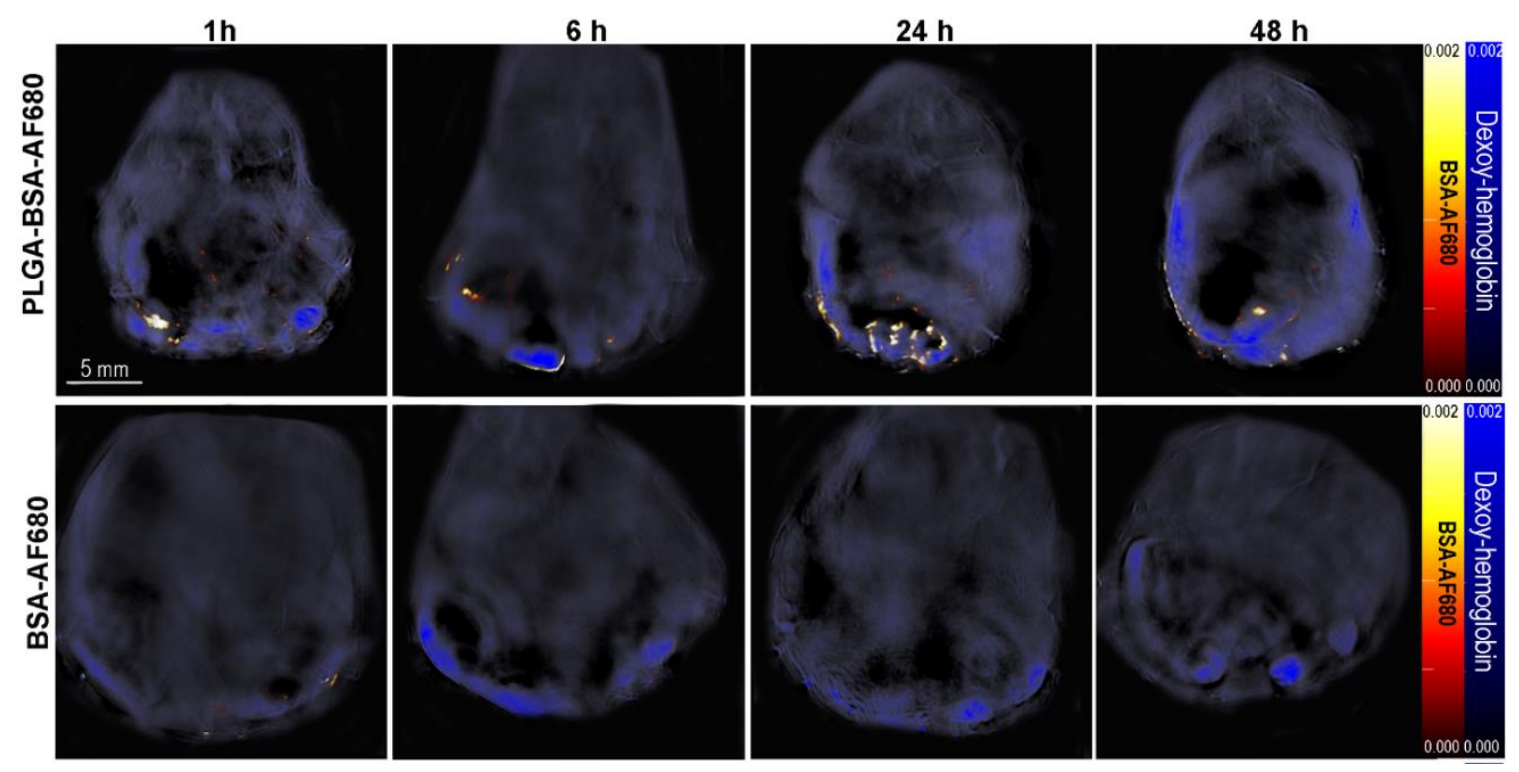

Figure 4: MSOT signal in gastrointestinal organs after oral gavage of either PLA-BSAAF680 particles or BSA-AF680 alone. (Top) MSOT imaging demonstrates nanoparticle localization and uptake in the proximal small bowel, distal small bowel, proximal colon, and mesenteric lymph node over the course of $48 \mathrm{~h}$. (Bottom) BSA-AF680 was undetectable after $1 \mathrm{~h}$ post gavage. Deoxy-hemoglobin is shown in blue and BSAAF680 in the hot color bar. All mouse images were equalized to the same intensity scale bar. 


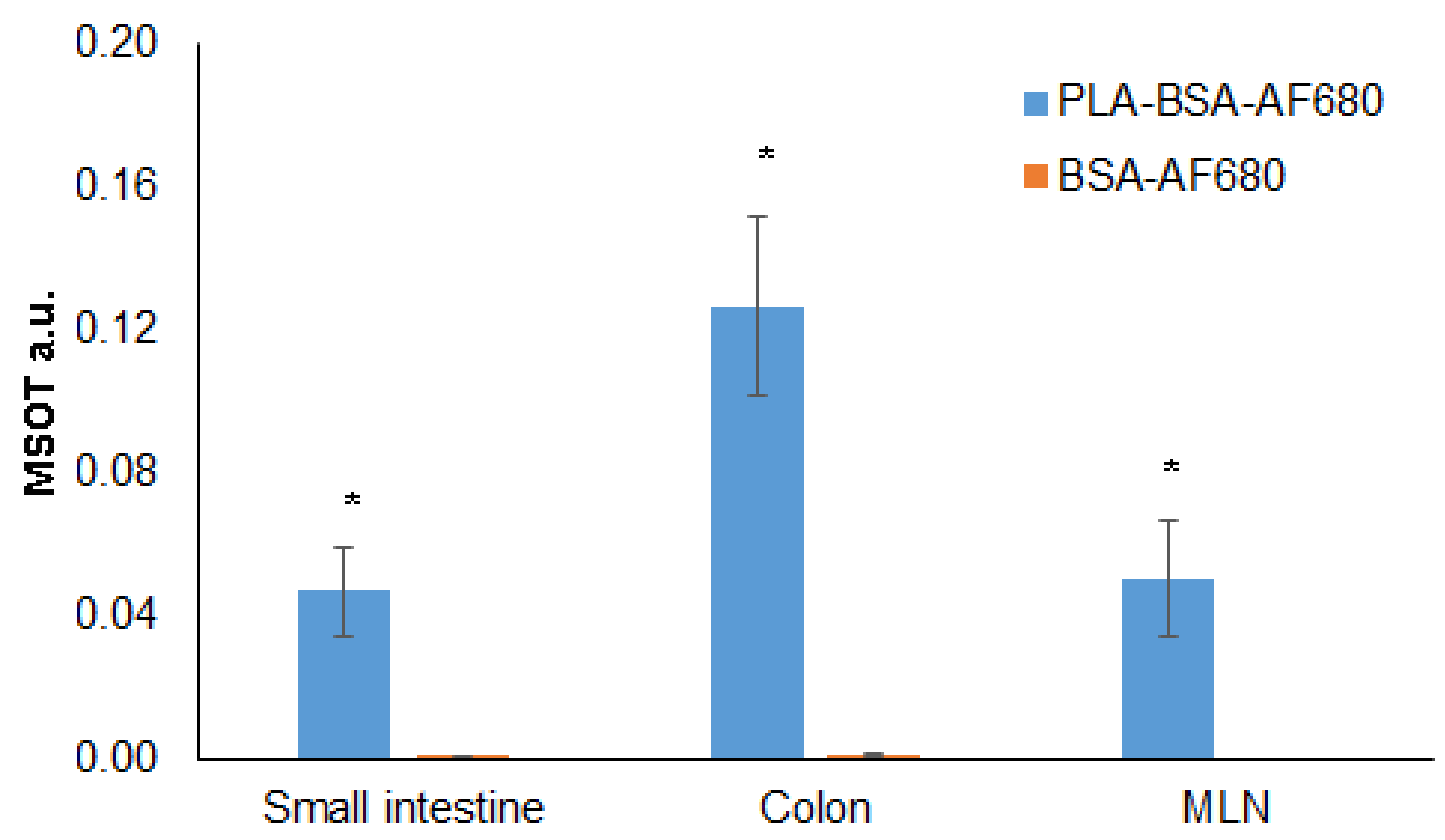

Figure 5: Mean MSOT signal intensity correlates with particle localization. Data shown here reflects mean MSOT signal intensity of PLA-BSA-680 or BSA-AF680 in colon, small bowel, and MLN 24 hours after gavage. Region of interest was determined using a 3.5 $\mathrm{mm}^{2}$ elliptical region on the assigned area of small intestine, colon, or MLN using View MSOT 3.5. Significantly higher levels of BSA-AF680 were observed in mice that received it via PLA particles than BSA-AF680 alone $(p<0.001)$. 

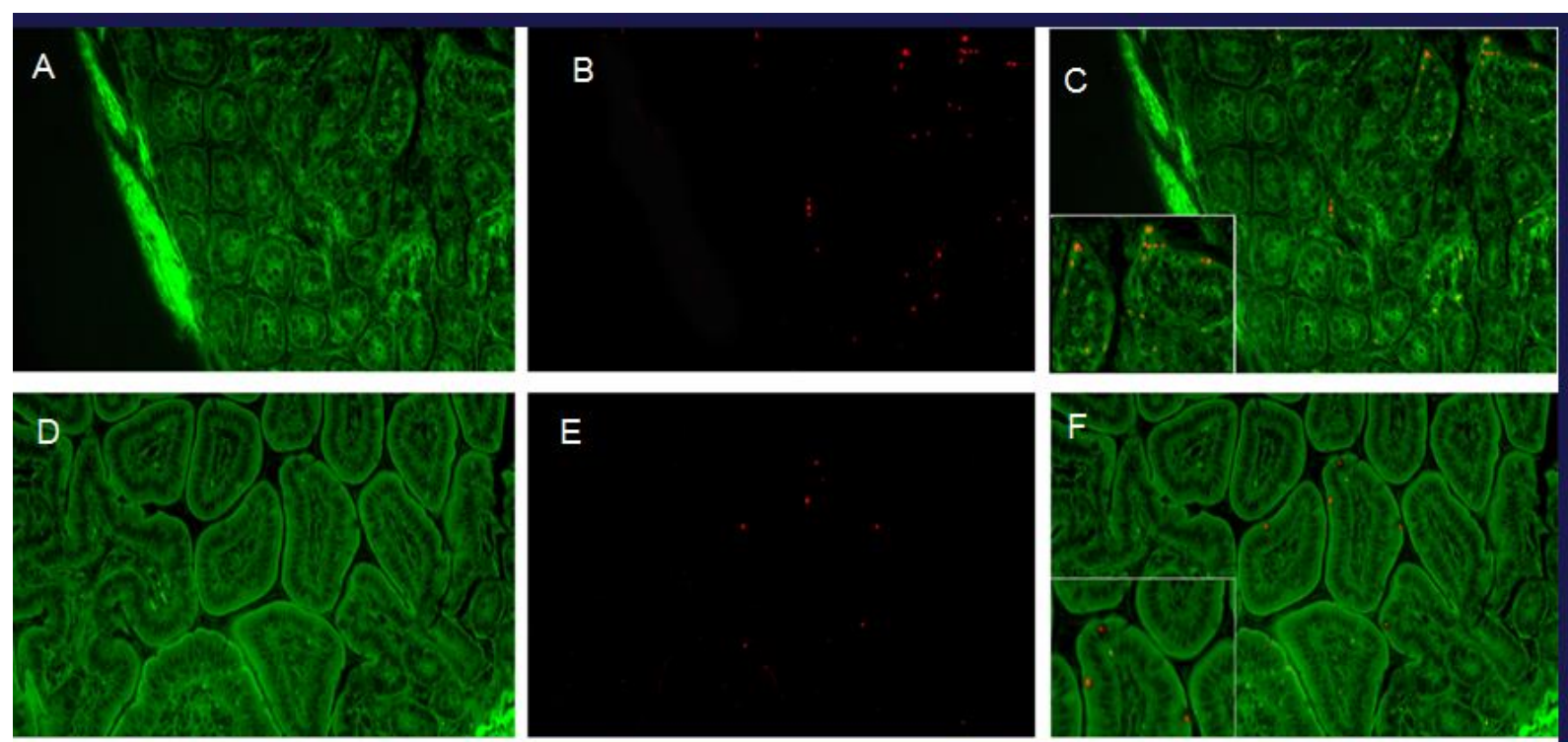

$\mathrm{E}$

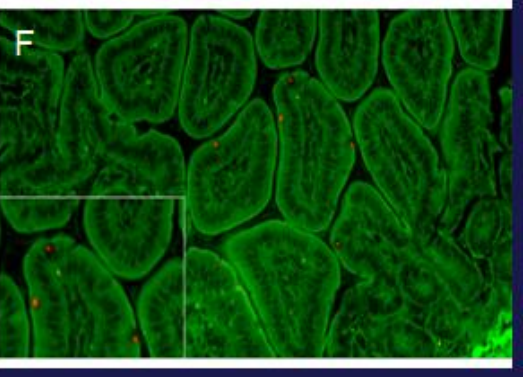

Figure 6: Histologic visualization of PLA-BSA-AF594. (A-C) demonstrates proximal colon 24 hours after oral gavage with PLA-BSA-AF594 (20x), with (C) showing an overlay of particles (red) on intestinal epithelium (green). (D-F) demonstrates small bowel 24 hours after oral gavage with particles containing AF-680 (20x), with (F) again showing an overlay of particles (red) on intestinal epithelium (green). 


\section{CHAPTER THREE}

TREATING COLON CANCER WITH ORAL IL-10 AND IL-12: THE SYNERGY MYSTERY

IDENTIFYING THE MECHANISMS BEHIND THE SYNERGISTIC EFFECT OF IL-10 AND IL-12 ON COLON TUMOR BURDEN AND DELINEATING THE EFFECT OF IL-10 AND IL-12 ON COLON EPITHELIAL BARRIER INTEGRITY AND ITS RELATIONSHIP TO THE EFFICACY OF ANTINEOPLASTIC THERAPY

\section{Introduction}

The recent clinical success of immune checkpoint inhibitors (ICI) represents a major breakthrough in cancer therapy. ${ }^{1}$ At the same time, the effectiveness of $\mathrm{ICI}$ has not been uniform across different solid tumor types. ${ }^{2}$ A major cancer type that remains highly resistant to $\mathrm{ICl}$, and immune therapy in general, is mismatch repair (MMR)proficient colorectal cancer (CRC). ${ }^{2,3}$ While the mechanisms underlying resistance of MMR-proficient CRC to immune therapy are not fully understood the need for the development of new therapeutic approaches is clear. ${ }^{3}$ 
One distinction between $\mathrm{CRC}$ and most other cancers is the unusual characteristics of the tissue within which the cancer arises. The colon represents a unique environment due to the massive commensal microbiota burden and has evolved complex mechanisms to maintain the delicate balance between lumenal bacteria and the immune cells that patrol the lamina propria (LP). ${ }^{4}$ Breach of the single-cell thick epithelial barrier that separates the lumen from the LP can result in the loss of intestinal immune homeostasis and the development of severe inflammatory pathology. ${ }^{5}$ Indeed, if such pathology becomes chronic, it can lead to development of cancer as seen in colitisassociated cancer. ${ }^{6}$ While inflammation that results from loss of epithelial barrier integrity can directly promote colon cancer, most sporadic CRC initially develops independent of chronic inflammation. However, once dysplasia develops, it will result in local compromise of the barrier and lead to what has been termed "tumor-elicited inflammation," a process that in turn promotes the growth of established adenomas. ${ }^{7}$ Therefore, chronic inflammation is tightly intertwined both with tumorigenesis and tumor progression in the colon. Consistent with this paradigm, a significant body of literature supports a major role for microbially-driven type $17 \mathrm{~T}$-cell immunity (including Th17 and үठT-cell subsets) in colon cancer. ${ }^{7-10}$ Importantly, analysis of clinical CRC samples have revealed an inverse relationship in CD8+ cytotoxic T-lymphocyte (CTL)/Th17 cell ratio between MMR-deficient (high CTL, low Th17) and ICl-resistant MMR-proficient (low CTL, high Th17) tumors ${ }^{11}$ in support of the notion that the outcome of immune therapy in CRC may be dependent on the ability to alter the CTL - Th17 cell balance.

Interleukin-10 (IL-10) is a pluripotent immune regulatory cytokine that is central to the maintenance of immune homeostasis in mucosal tissues. ${ }^{12} \mathrm{IL}-10$ converts immature blood monocytes to tolerogenic macrophages, ${ }^{13-15}$ has direct suppressive effects on 
Th17 cell activity, ${ }^{16}$ and conversely enhances conventional regulatory T-cell (cTreg) function. ${ }^{17}$ A series of recent studies demonstrated that IL-10 directly activates antigenexperienced CD8+ cytotoxic T-lymphocytes independent of its suppressive effects on CD4+ T-effector and/or myeloid cell subsets. ${ }^{18-20}$ Collectively, these properties have provided the rationale for our studies evaluating the therapeutic efficacy of a novel oral formulation of IL-10 in the treatment of intestinal polyposis ${ }^{21}$ and, more recently, of colon cancer. ${ }^{22}$ The findings revealed that oral IL-10 could suppress tumorigenesis in the above models via its dual activity on Th17 cells and CTL.

In this study, we tested whether oral IL-10 would be effective in eradicating established disease either alone or in combination with IL-12, a canonical Th1 cytokine that can directly activate tumor-associated CTL. ${ }^{23}$ The results demonstrate potent synergy between IL-10 and IL-12, involving pleiotropic effects on immune cells and the gut epithelium, with the latter activity being critical to overall therapeutic efficacy.

\section{Materials and Methods}

Mice and the tumor model. C57BL/6 (B6), C57BL/6J-Apc ${ }^{\mathrm{Min}} / \mathrm{J}\left(\mathrm{APC}^{\mathrm{Min} /+}\right)$ and B69SJL)IL-10ra ${ }^{\mathrm{tm} 1.11 \mathrm{lg} / \mathrm{J}}$ (IL-10RA knockout) mice were purchased from Jackson Laboratory. Enterotoxic $B$ fragilis strain 86-5443-2-2 was a kind gift from Dr. Cynthia L Sears (Johns Hopkins University School of Medicine, Baltimore, Maryland). For colonization with $B$ fragilis, 5-6 week old APC ${ }^{\mathrm{Min}+}$ mice were administered clindamycin $(0.1 \mathrm{~g} / \mathrm{L})$ and streptomycin $(5 \mathrm{~g} / \mathrm{L})$ ad libitum in drinking water for 5 days before oral gavage $\left(\sim 5 \times 10^{7}\right.$ bacteria in PBS) as previously described. ${ }^{8}$ All studies were conducted in accordance with guidelines set forth by the Institutional Animal Care and Use Committee at the University of Louisville (Louisville, KY). 
Reagents and treatments. Two particle formulations were produced using a modified Phase Inversion Nanoencapsulation (PIN) process: ${ }^{37}$ (i) control (no cytokine) and (ii) recombinant murine IL-10 or IL-12-encapsulated (Peprotech, Inc.) with a loading of 0.5 $\mu \mathrm{g}$ or $0.25 \mu \mathrm{g}$ cytokine/mg of particles, respectively. Particles were administered via oral gavage ( $1 \mathrm{mg}$ particles in $0.2 \mathrm{~mL}$ sterile water for blank, IL-10, and IL-12 treatments and $2 \mathrm{~mL}$ total particles in $0.2 \mathrm{~mL}$ sterile water for combination IL-10 and IL-12 treatment) starting 4 weeks after $B$ fragilis inoculation three times per week for 3 weeks. An experimental schematic is shown in Figure 1. Administration of particles resulted in a transient but significant increase in cytokine levels in the gut lamina propria (Figure 2). For survival analysis, mice were treated until they reached the IACUC-approved euthanasia score as previously described by our group. ${ }^{21}$

Gross intestinal preparation and tumor quantification. Colons were opened longitudinally before being fixed in $10 \%$ neutral buffered formalin. Tumor burden was quantified using a dissecting microscope.

Histology. Formalin-fixed, parrafin-embedded tissue from the distal colon was sectioned

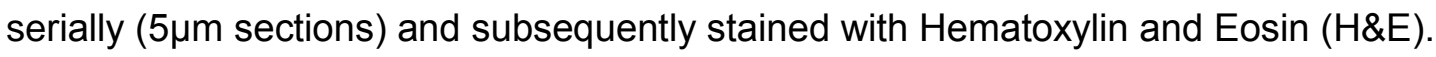
Colon histology was assessed in a blinded fashion by a single tumor pathologist. Each section was classified as harboring no dysplasia, low grade dysplasia, or high grade dysplasia. Colons were then scored according to the following system based on the average severity of dysplasia in the distal colon: 0 - no dysplasia; 1 - low grade dysplasia only; 2 - mixture of low grade and high grade dysplasia; 3 - high grade dysplasia only; 4 - invasive cancer. 
Laser-scanning Confocal microscopy. Colon and tumor tissues were harvested from mice, embedded in Tissue-Plus Optimal Cutting Temperature (OCT) Compound (Fisher HealthCare, Houston, TX, USA) and snap-frozen in liquid nitrogen. Serial cryosections $(25 \mu \mathrm{m})$ were prepared with a Cryostar NX70, Thermo Scientific cryostat at $-19^{\circ} \mathrm{C}$ (Kalamazoo, MI, USA). Cryosections were kept at room temperature for at least $24 \mathrm{~h}$ prior to staining. A previously described immunostaining protocol was used with modifications. ${ }^{38}$ For analysis of IL-10RA expression, staining antibodies were added sequentially in the following order: IL-10RA- phycoerythrin (PE) (Novus Biologicals, Littleton, CO), CD324 (E-Cadherin) Alexa Fluor-488 (Thermo Fisher, Waltham, MA). Sections were washed twice with 1X PBS-T and processed for imaging. For analysis of colon sections, staining antibodies were added sequentially in the following order: IL10RA- phycoerythrin (PE) (Novus Biologicals, Littleton, CO), CD324 (E-Cadherin) Alexa Fluor-488 (Thermo Fisher, Waltham, MA). Antibodies were diluted with $2 \%$ fetal calf serum (FCS) in 1X PBS pH 7.4 to 1:25 for IL-10RA-PE, and 1:25 for CD324 E-Cadherin Alexa Fluor-488. Each antibody was sequentially incubated at $37^{\circ} \mathrm{C}$ for 40 mins. Sections were washed twice with 1X PBS-T and Prolong Gold anti-fade reagent (Thermo Fisher, Waltham, MA) was added to the slides prior to imaging. Images were captured using a Leica SP5 confocal laser scanning microscope (Leica, Wetzlar, Germany) and processed using Fiji Software. ${ }^{39}$ Panels containing confocal images were generated using Adobe Photoshop version 13.0 ×32. Images were marked using the drawing tools to highlight the results and to provide orientation of the tissues.

Colon epithelial cell isolation. Mouse (C57BL/6) colons were excised, flushed with PBS + penicillin and streptomycin (P/S), hemisected longitudinally, and rinsed with ice cold PBS + P/S. Colons were then cut into $5 \mathrm{~mm}$ pieces and placed in a $50 \mathrm{~mL}$ conical tube containing $20 \mathrm{~mL} \mathrm{HBSS}+1 \mathrm{mM}$ DTT + $1 \mathrm{mM}$ EDTA + 5\% FBS. Tubes were placed in a 
hybridization oven and incubated at $200 \mathrm{RPM}, 37^{\circ} \mathrm{C}$ for 40 minutes. Contents were then passed through a $100 \mu \mathrm{m}$ filter and centrifuged at 1500 RPM for 5 minutes. Supernatant was discarded, and the pellet was subjected to density dependent centrifugation using a $25 \% / 40 \%$ Percoll gradient. Cells were harvested at the interface of the solutions and placed in $2 \mathrm{~mL}$ 100\% FBS. Cells were centrifuged again at 1500 RPM for 5 minutes and reconstituted in $2 \mathrm{~mL}$ PBS + 0.1\% BSA for FACS analysis.

Flow cytometry. Membrane and intracellular staining of MLN or epithelial cells were performed as described. ${ }^{22}$ The following antibodies were used: CD4 (GK1.5, BioLegend), CD8 $\alpha$ (53-6.7, BD Biosciences), CD16/CD32 (93, BioLegend), IL-17A (TC11-18H10.1, BioLegend), RORyt (Q31-378, BD Biosciences), IFNy (XMG1.2, BD Biosciences), IL-10RA (1B1.3a, BioLegend), and Ep-CAM (G8.8, BioLegend). Lymphocyte depletion and functional blockade studies. Anti-mouse CD8a (53-6.72, BioXCell) was given intraperitoneally (ip) to $\mathrm{APC}^{\mathrm{min} /+}$ mice $(0.2 \mathrm{mg}$, three times per week for 3 weeks) to deplete CD8+ T lymphocytes. Anti-mouse IFNy (XMG1.2, BioXCell) was injected ip $(0.2 \mathrm{mg}$, three times per week for 3 weeks). All treatments were initiated on treatment day -1 (the day before receiving their first oral immunotherapy treatment) and again on treatment day 0 (the day of their first oral immunotherapy treatment). Mice were subsequently treated IP twice weekly for the duration of their 3 week oral immunotherapy treatment regimen.

Colon permeability study. Colon permeability was assessed using a FITC-dextran assay as previously described. ${ }^{40}$ Briefly, $\mathrm{APC}^{\mathrm{min} /+}$ / $B$ fragilis mice were treated with oral immunotherapy as described above. After 3 weeks of treatment, they were water starved overnight before being gavaged with $44 \mathrm{mg} / 100 \mathrm{~g}$ body weight of FITC labeled 
dextran (FD4, Millipore Sigma, St. Louis, Missouri, USA) suspended in sterile PBS at a concentration of $100 \mathrm{mg} / \mathrm{mL}$. After a period of 4 hours, $300 \mathrm{~mL}$ of blood was extracted retro-orbitally and placed in a BD SST collection tube (BD, Franklin Lakes, New Jersey, USA). After centrifugation, serum was aspirated and diluted 1:1 with sterile PBS.

Samples were pipetted into a 96-well plate and analyzed using a plate reader (em: 485 $\mathrm{nm}$, ex: $526 \mathrm{~nm}$ ). Concentration of FITC-dextran was calculated based upon a standard curve.

Colon explant culture. Colon tissue pieces $\left(0.5-1 \mathrm{~cm}\right.$ length) from $\mathrm{APC}^{\mathrm{min}+} / \mathrm{B}$ fragilis mice were cultured in triplicates for 24 hours in complete DMEM-high glucose medium (supplemented with $10 \%$ fetal bovine serum, $1 \mathrm{X}$ penicillin-streptomycin solution) in a humidified atmosphere $\left(37^{\circ} \mathrm{C}, 5 \% \mathrm{CO}_{2}\right)$ in the presence of recombinant murine $\mathrm{IL}-10(30$ ng/mL, PeproTech, Rocky Hill, New Jersey, USA), recombinant murine IFNY (20 ng/mL, PeproTech, Rocky Hill, New Jersey, USA), or a combination of recombinant murine IL10 and IFNY. The tissues were processed for protein preparation (tissue lysates with RIPA buffer) using a sonic dismembrator (Model 550, Fisher Scientific). These tissue lysates were used to determine the expression of IL-10RA, claudin-4, and occludin.

Western blots. Total protein lysates were collected either from colon tissue or colon epithelial cells as described above using radioimmunoprecipitation assay (RIPA) buffer (Millipore Sigma, St. Louis, Missouri, USA) and quantified using BCA protein quantification kit (Thermo Fisher Scientific, Waltham, Massachusetts, USA) per instructional manual. Total protein $(20-50 \mu \mathrm{g})$ of was resolved on Mini-PROTEAN TGX 4-20\% gels (Bio-Rad, Hercules, California, USA) and transferred to polyvinylidene difluoride membrane (0.22 $\mu$ m pore; Novex, Carlsbad, California, USA). After blocking with $3 \%(w / v)$ bovine serum albumin (BSA) (containing $1 \mathrm{X}$ TBS) for $1 \mathrm{~h}$, the membrane 
was then incubated with HRP-conjugated anti-claudin-4, anti-occludin, anti-IL-10RA and anti- $\beta$-actin antibodies (1:500, 1:500, 1:300 and $1: 20,000$ dilution, respectively) at $4{ }^{\circ} \mathrm{C}$ overnight. For all proteins, chemiluminescent substrate (SuperSignal ${ }^{\mathrm{TM}}$ West Femto Maximum Sensitivity Substrate, Thermo Scientific, Rockford, Illinois, USA) was used to detect the protein bands (ImageQuant LAS 4000). Densitometry analysis of bands was done using ImageJ software. Antibodies for claudin-4, occludin and $\beta$-actin were purchased from Santa Cruz Biotechnologies (USA). The antibody for IL-10RA was purchased from Novus Biologicals (USA).

Quantitative PCR. Steady-state mRNA levels in colon tissue were detected with SYBR Green PCR Master Mix (Applied Biosystems) using the Mx3000p qPCR system (Agilent Technologies). Results were normalized to $\beta$-actin expression. The expression level was scaled using the $2^{-\triangle \Delta C T}$ method, with the average levels obtained for colons of APC $\mathrm{min} /+$ / $B$ fragilis mice treated with blank (control) microparticles set arbitrarily to 1 . Primer sequences utilized were: $\beta$-actin forward 5'-TCACCCACACTGGCCCATCTACGA-3', reverse 5'-TGGTGAAGCTGTAGCCACGCT-3'; IL-10RA forward 5'GCCAAGCCCTTCCTATGTGT-3', reverse 5'-CCAGGGTGAACGTTGTGAGA-3'; IFNy forward 5'-GGCACAGTCATTGAAAGC-3', reverse 5'-TGCCAGTTCCTCCAGATA-3'; claudin-4 forward 5'-ATGGCGTCTATGGGACTACA-3', reverse 3'TTACACATAGTTGCTGGCGG-5'; occludin forward 5'- CCTCCAATGGCAAAGTGAAT3', reverse 3'- CTCCCCACCTGTCGTGTAGT-5'.

Statistical Analysis. Two-tailed student's t-test was used to determine the significance of the differences between control and experimental groups in pairwise comparisons. In experiments with multiple groups homogeneity of inter-group variance was analyzed by one-way ANOVA with Tukey's honest standard difference for multiple comparisons. 
Log-rank test was utilized for analysis of survival studies. $P$ values of 0.05 or less were considered statistically significant. Statistical analyses were performed using GraphPad Prism 7 (GraphPad Software, La Jolla, California, USA) and MedCalc version 17.9.7 (MedCalc Software, Ostend, Belgium).

\section{Results}

Previous studies demonstrated that oral administration of a slow-release biodegradable particulate formulation of IL-10 suppressed intestinal polyposis in APC ${ }^{\mathrm{min} /+}$ mice and early tumorigenesis the APC ${ }^{\mathrm{min} /+} / B$ fragilis compound colon cancer model. ${ }^{21,22} \mathrm{We}$ wanted to determine whether this approach would be effective in the treatment of established disease. To this end, APC ${ }^{\mathrm{min} /+}$ mice were inoculated with $B$ fragilis and were allowed to develop tumors prior to the initiation of therapy. They were then administered IL-10 for 3 weeks, and colon tumor burden as well as histology were analyzed. The results demonstrate that short-term treatment reduced tumor burden by $50 \%$ (Figure 3A) coupled with a modest decrease in maximum tumor diameter (Figure 3B). In contrast, treatment did not affect tumor histopathology (Figure 3C).

IL-10 suppresses colon tumorigenesis via its ability to reduce the prevalence of IL-17producing T-cells and concurrently enhance of CTL cytotoxicity. ${ }^{22}$ We therefore hypothesized that adding IL-12 to the treatment regimen could further augment the functional balance in favor of CTL. To test this notion, mice with established disease were treated with each cytokine separately or the two cytokines in combination. Analysis of tumor burden in mice that received monotherapy confirmed the beneficial activity of IL-10 while IL-12 was found to increase average tumor number by approximately $30 \%$ (Figure 4A). In contrast, combined therapy achieved near-complete 
tumor elimination in the majority of mice (Figure 4A). A similar trend was observed with regard to tumor size where combination therapy mediated a significant $30 \%$ reduction in maximum tumor diameter. Importantly, and in contrast to treatment with IL-10 alone, histological analysis revealed a dramatic improvement in the pathological score of tumors in mice that received combination therapy (Fig 4B).

The above findings suggested that combined therapy not only arrested tumor growth but actively promoted eradication of established disease. To determine whether combined therapy could provide long-term benefit in this aggressive carcinoma model, mice with established tumors were treated continuously with IL-10 and IL-12 in a survival study. The data shown in Fig 4C demonstrate a 30\% increase in median survival in the treatment (93 days) vs the control (71 days) group. Importantly, approximately $30 \%$ of the experimental mice remained alive up to and beyond 140 days post-initiation of treatment ( 210 days of age), exceeding the maximum lifespan of the APC ${ }^{\mathrm{min} /+}$ mouse..$^{21}$

Next, we wanted to delineate the cellular mechanism(s) that were responsible for the synergy. Quantitative as well as qualitative analysis of MLN T-cell populations were performed in control vs. treatment groups. Analysis of the IL-10 alone group demonstrated a $35 \%$ reduction in the number of CD4+RORyt+IL-17+ Th17 cells with no significant impact on CD8+ T-cell activity (Figure 5A). IL-12 monotherapy did not have a detectable effect on Th17 cell numbers but enhanced CTL prevalence and activity. Importantly, combination therapy reduced Th17 cell numbers and increased CTL activity, enhancing the CTL to Th17 cell ratio (Figure 5A). These data demonstrate that each cytokine modulated distinct effector mechanisms in gut-associated immune structures with minimal cross-antagonism. 
To determine whether the observed synergy between IL-10 and IL-12 was simply due to enhanced CTL activity in the presence of reduced Th17 prevalence, combination treatment was performed in the presence or absence of CD8+ T-cell depletion. Analysis of tumor burden revealed that depletion of CD8+ T-cells indeed resulted in reduced antitumor efficacy, though this loss was partial and did not reach statistical significance (Figure 5B). This finding, in combination with the independent observation that IL-12 alone actually worsened disease burden, suggested additional mechanisms underpinning the observed synergy.

IL-12 mediates its immunological activity primarily via its immediate downstream effector IFNy. ${ }^{23}$ To obtain further insight into the dichotomous effects of IL-12 in our model, we first investigated the requirement for IFNy in the pro- vs anti-tumorigenic activity of IL-12 when administered alone or in combination with IL-10, respectively. In vivo neutralization of IFNy in the control and experimental groups demonstrated that blockade of IFNy activity resulted in the abrogation of both the detrimental and the beneficial activities of IL-12, confirming that both pathways required IFNy signaling (Figure 6A). This finding suggested that in the combination therapy setting, cooperation between IFNy and IL-10, two cytokines that are traditionally thought to be antagonistic, was responsible for the unexpected synergy.

In addition to their direct effects on immune effectors, IL-10 and IFNy are known to reciprocally modulate the paracellular physiology of gut epithelium, ${ }^{24}$ with potential impact on pro-tumorigenic inflammatory processes. Specifically, in the APC ${ }^{\mathrm{min}+/}$ model, modulation of gut permeability by DSS ${ }^{25}$ results in exacerbation of inflammatory activity and promotes tumorigenesis in the colon. ${ }^{26}$ Therefore, to determine whether the IL-10and/or IL-12-IFNy-epithelial barrier axis played a role in the observed synergy, we 
undertook examination of gut epithelial barrier function in different treatment groups. Mice were fed FITC-labeled dextran particles, and serum levels of particles were determined to assess gut permeability in each group. The data show that IL-10 slightly reduced whereas IL-12 substantially enhanced (by 3-fold) permeability compared to that in control mice (Figure 6B). In contrast, co-administration of IL-10 with IL-12 restored serum FITC-dextran levels to steady-state, providing direct evidence that combination therapy had significant impact not only on immune cells but also on the integrity of the gut epithelium. Importantly, neutralization of IFNy during treatment abrogated the detrimental effect of IL-12 on barrier integrity, mechanistically linking the effects of the IL12-IFNy axis on barrier integrity and tumor progression (Figure 6, panels $A$ and $C$ ).

We next addressed the mechanism underlying the ability of IL-10 to restore epithelial barrier function in IL-12-treated mice. IL-10 is known to enhance tight junction protein expression in the gut epithelium. ${ }^{24,27-29}$ Separately, IFNy was recently shown to induce IL-10RA expression on intestinal epithelial cells. ${ }^{30}$ We therefore hypothesized that sensitization of colon epithelium to IL-10 by the IFNY-IL-10RA axis could be responsible for restoration of barrier integrity in mice receiving dual therapy. To this end, we first determined whether oral IL-12 altered IL-10RA expression in the gut. Quantitative PCR analysis revealed that IL-12 promoted 3- and 6-fold increases in IFNy and IL-10RA mRNA expression in the colon, respectively; whereas IL-10 alone had no significant effect (Figure 7A). To determine whether IL-10RA was upregulated on the colon epithelium, we analyzed colon tissue from control and experimental groups by confocal microscopy. The data showed robust IL-10RA expression in the colon epithelium in mice treated with IL-12 or IL-12 + IL-10, whereas no significant protein could be visualized in the control or IL-10 only groups (Figure 7B). We then quantitatively assessed IL-10RA expression on colonic epithelial cells of control vs experimental mice 
by FACS analysis. These data revealed an approximately 3-fold increase in IL-10RA+ epithelial cells in the colons of mice that received IL-12 particles (Figure 7C).

Collectively, these results supported the hypothesis that restoration of barrier integrity in mice receiving dual treatment vs IL-12 alone was associated with increased responsiveness of IFNy-conditioned epithelium to exogenous IL-10.

We further pursued the above hypothesis using an in vitro colon explant culture system in which the predicted mechanism could be assessed directly. Specifically, we evaluated the effect of cytokine exposure on select tight junction protein levels. To this end, colons of $B$ fragilis-infected APC ${ }^{\mathrm{min} /+}$ mice were incubated in media or media with IL10, IFNy or IL-10 + IFNy; and epithelial occludin, claudin-4 and IL-10RA expression were quantified by qPCR and Western blot. The results demonstrated that IFNy, alone or in combination with IL-10, enhanced IL-10RA transcript levels by 8 to 10 -fold on average (Figure 8A). Similar increases in both occludin and claudin-4 mRNA were also observed, but only in the combination group (Figure 6A). Western blot analysis revealed a similar trend in protein expression in explants that were exposed to IFNy + IL-10 for all markers (Figure 8B). Collectively, these findings further supported the mechanistic hypothesis that the stromal effect of the cytokines was associated with enhanced tight junction integrity, which required IFNy-dependent sensitization of the epithelium to IL-10 (Figure 9).

\section{Discussion}

Herein, we demonstrate that oral delivery of IL-10 and IL-12 can effectively eradicate established tumors in the APC ${ }^{\mathrm{min} /+}$ / B fragilis colon cancer model. Further, we provide mechanistic insight into the mechanisms that underlie the synergy between IL-10 and IL- 
12, two conventionally-antagonistic cytokines. Specifically, we found that, in addition to their distinct immunological effects on Th17 and CTL activity, combined administration of IL-12 and IL-10 improved gut barrier integrity via the IFNy-IL-10RA axis, greatly enhancing therapeutic outcome. These findings have important clinical implications for immune-based therapy of colon cancer, which has traditionally been resistant to this modality.

The primary immunological effects of IL-10 and IL-12 involved distinct activities on Th17 and CD8+ T-cell activity, respectively. Specifically, IL-10 diminished the prevalence of IL-17-producing CD4+ RORyt+ Th17 cells consistent with our previous observations. ${ }^{21,22}$ IL-12, on the other hand, enhanced IFNy-producing CD8+ T-cell numbers. Importantly, these activities were independent and were not antagonistic. We hypothesize that the lack of antagonism between the two cytokines was associated with the distinct and in some cases opposing effects of IL-10 on T-cells of different ontogeny and maturation stage. Specifically, while IL-10 is known to globally suppress T-effector cell priming via its tolerogenic activity on antigen-presenting cells, ${ }^{12-15}$ recent data are consistent with differential effects on antigen-experienced effector subsets. For example, while IL-10 can directly suppress Th17 effector cell expansion, ${ }^{16}$ multiple reports have confirmed its ability to enhance the activity of primed CD8+ T-cells. ${ }^{18-20,22}$ Conversely, the suppression of IL-10 production by IFNy, the downstream effector of IL-12, occurs at the level of transcription, ${ }^{31}$ and delivery of recombinant IL-10 would bypass such a mechanism. It is therefore likely that these selective effects on terminally-differentiated T-effector cell subsets underlie the lack of immunological antagonism between the two cytokines in our system. 
An unexpected finding in this study was that the sensitization of the gut epithelium to IL10 by IL-12 was ultimately responsible for the greater part of the synergistic antitumor effect. Whereas our data demonstrate a link between barrier function and therapeutic outcome, they do not directly address the mechanistic basis of how loss of barrier integrity led to increased tumor growth. It is well-known that disruption of gut epithelial barrier can promote tumor growth via complex multi-pathway inflammatory processes that involve both microbial as well as non-microbial factors. ${ }^{32,33}$ Specifically, both type 17 and type 1 T-cell activity, as well as innate inflammatory cells can contribute to tumor growth in the inflamed gut. ${ }^{32,33}$ In the case of the APC ${ }^{\mathrm{min} /+} / B$ fragilis model, microbiallydriven type 17 immunity has been shown to be essential to tumor development. ${ }^{8,22,34}$ While our findings are consistent with this paradigm, the ability of IL-12 to exacerbate disease without significant impact on Th17 cells suggests that additional, yet unidentified, factors that are associated with increased gut permeability may also contribute to tumor pathogenesis in this model.

Our data provide partial insight into the molecular mechanism(s) that underlie the combined effects of IL-12 and IL-10 on barrier function. Consistent with previous reports, IL-12, through its downstream effector IFNy, upregulated IL-10RA expression in the colon epithelium in vivo and in vitro. Importantly, combined treatment of colon explants with IFNy + IL-10 induced the expression of tight junction proteins occludin and claudin-4. Collectively, these observations support the hypothesis that increased IL-10 signaling in the IFNy-conditioned gut epithelium ultimately restored barrier integrity via enhanced tight junction formation. In this study, we examined only two tight junction proteins that are known to be critical to barrier function. Given the variety and the complexity of tight junction protein family, a more detailed analysis of the global changes in tight junction protein levels as well as spatial localization could further delineate the 
molecular pathways underlying the observed effect. A question that remains is how IL10 signaling overcame the detrimental activity of IFNy in the combination therapy group. IL-10 and IFNy can cross-modulate each other's signaling pathways. ${ }^{30,35}$ Whether the dominance of one over the other is determined simply via the relative strength of STAT1 vs STAT3 signaling, and/or through additional pathways remains to be determined.

The above findings demonstrate that colon physiology can be effectively modulated by orally-administered slow-release cytokine formulations, establishing further proof-ofprinciple for the clinical potential of this therapy. This strategy provides the advantages that drugs can be delivered in a tissue-specific manner to achieve sustained physiological levels in the disease microenvironment with minimal systemic toxicity. We have, in the past, demonstrated similar success with oral cytokine formulations in models of IBD, intestinal polyposis and colon cancer. ${ }^{21,22,36}$ The current data not only confirm and extend previous findings but also further identify a novel therapeutic modality involving the synergistic use of two traditionally antagonistic cytokines. 


\section{Figures}

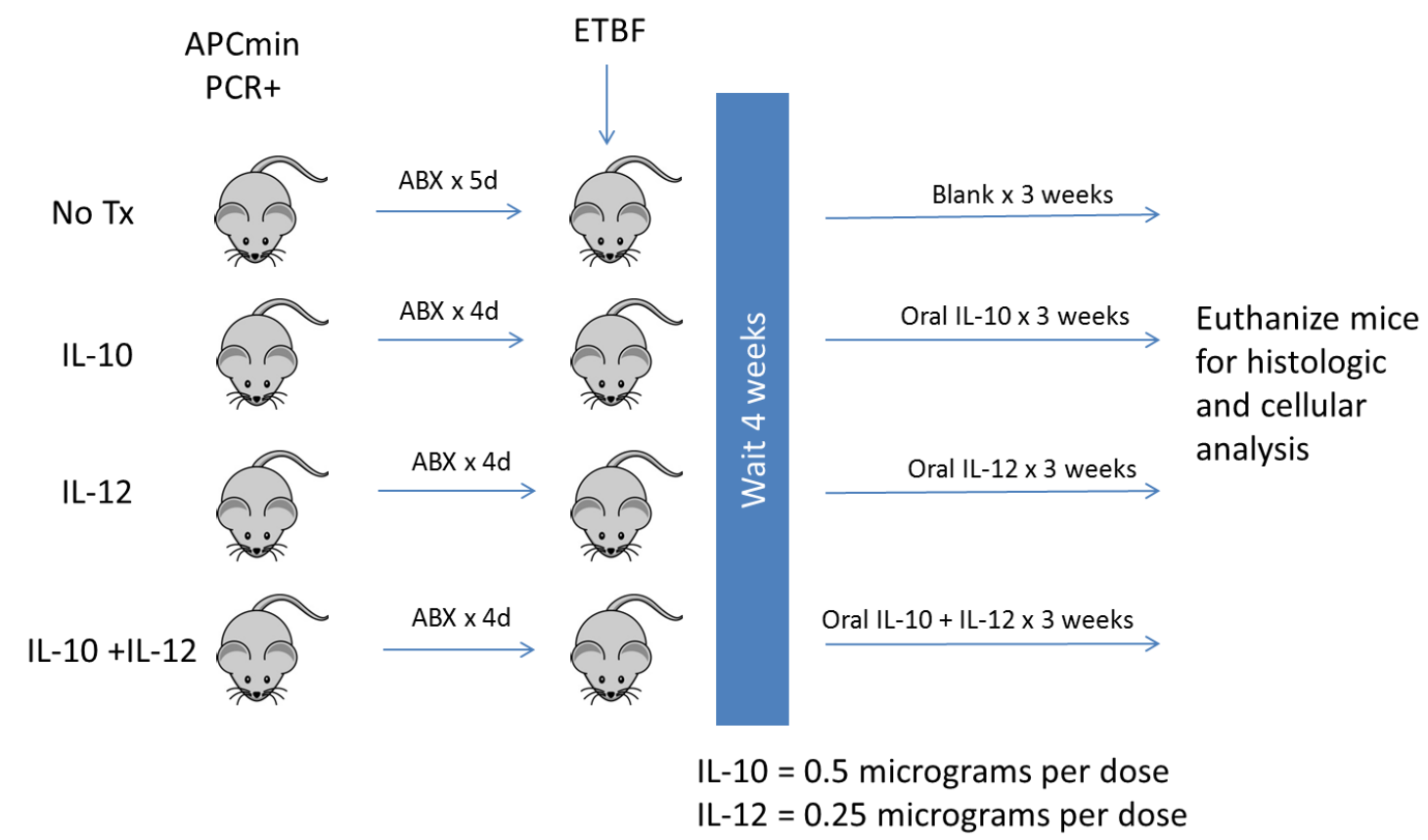

Figure 1: Experimental schematic 


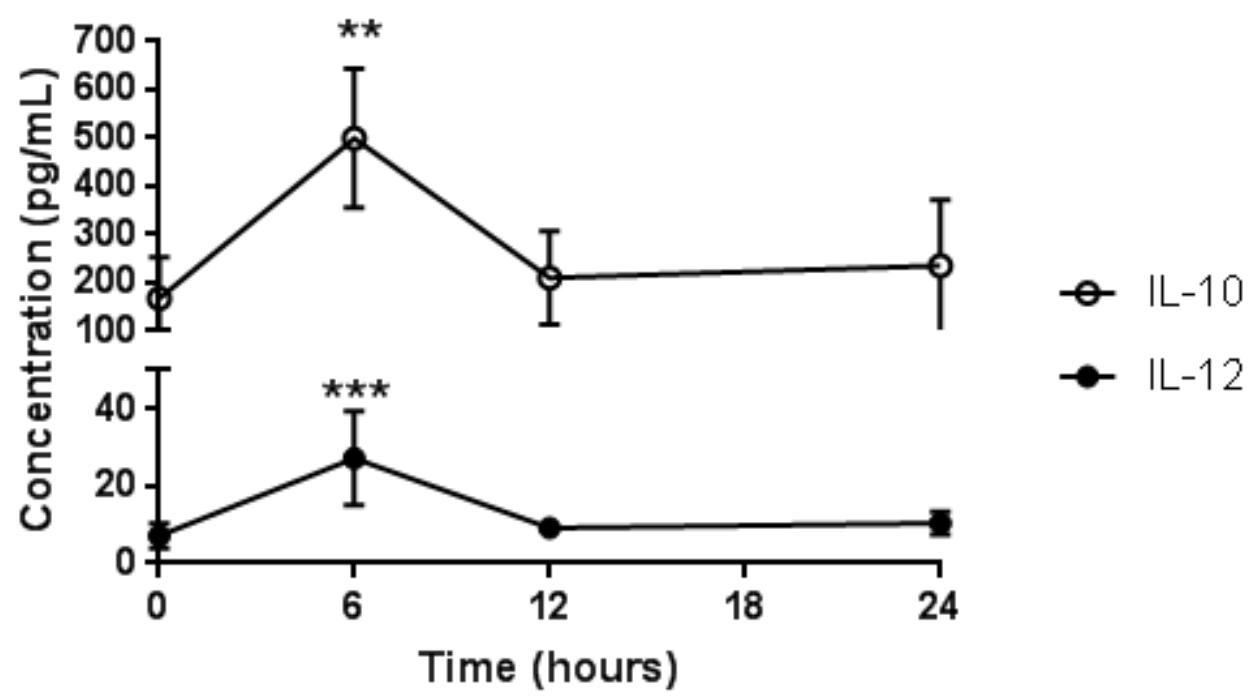

Figure 2: Oral delivery of cytokines to gut lamina propria. Mice (C57BL/6) were administered a single dose of IL-10 and IL-12 particles (10 $\mathrm{mg}$ of each). Distal small bowel, cecum, and proximal colon were harvested prior to particle administration (time 0) as well as 6,12 , and 24 hours after particle administration. Tissues were flushed with ice cold PBS + P/S and placed in $1 \mathrm{~mL} 2 x$ cell lysis buffer (Cell Signaling Technology, Danvers, MA) containing $1 \mathrm{mM}$ PMSF and were homogenized using a disposable Biomasher II Closed System Microtissue homogenizer (Kimble Chase Life Science, Rockwood, TN) followed by sonication (Model 550, Fisher Scientific) for 20 seconds. Homogenates were then incubated on ice for 20 minutes, centrifuged for 20 minutes at $12,000 \times \mathrm{g}$ and supernatants were analyzed for IL-10 and IL-12 by enzyme-linked immunosorbent assay (ELISA) using the LegendMax mouse IL-10 and mouse IL-12p70 ELISA kits (BioLegend, San Diego, CA). Error bars $=S D, n=5$ mice/time point. Statistical significance: ${ }^{* *}$ and ${ }^{* *}$ denote $p<0.01$ and 0.001 , respectively. 
A

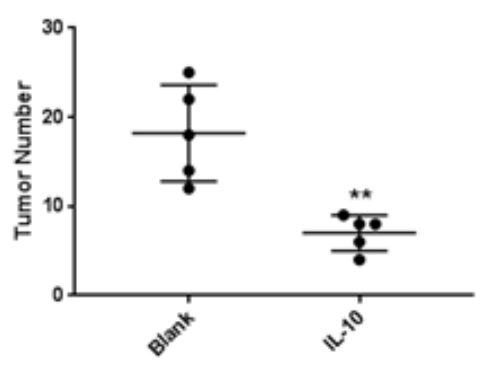

C

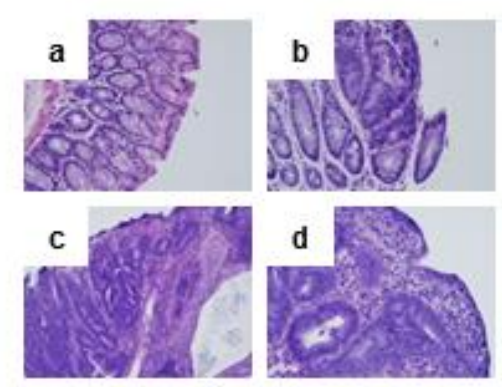

$\mathrm{B}$
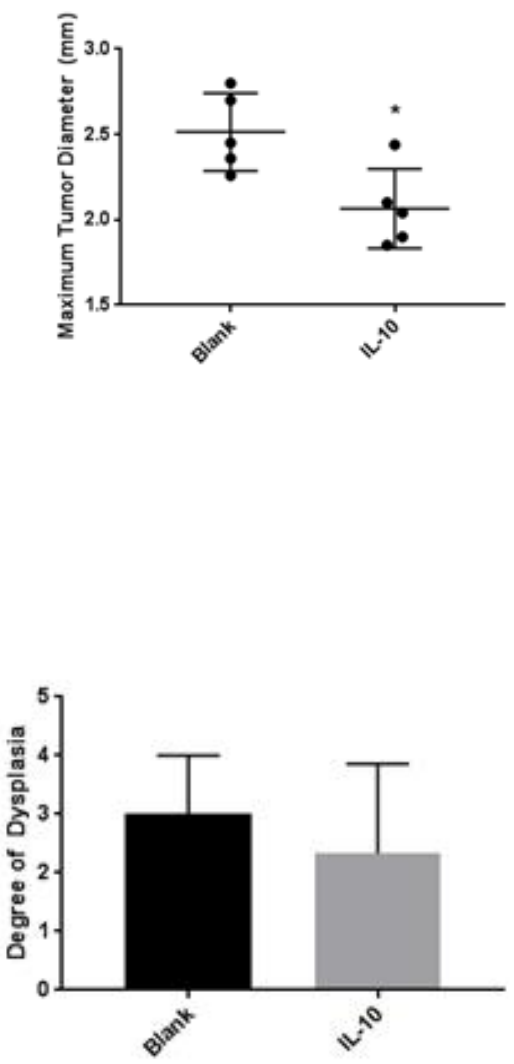

Figure 3: Oral IL-10 reduces tumor burden in mice with established disease. (A, B) Colon tumor number and maximum diameter. $\mathrm{APC}^{\mathrm{min} /+}$ mice were treated with oral particle-based therapy (either blank particles or particles loaded with recombinant murine IL-10) for 3 weeks beginning 4 weeks after enterotoxic $B$ fragilis inoculation. Mice were then euthanized, and tumor number $(A)$ and maximum tumor diameter $(B)$ in the mouse colon were assessed. Error bars $=S D, n=5$ per group. ( $C, D)$ Histologic severity of disease. At the time of euthanasia, colons were fixed in $10 \%$ neutral buffered formalin, embedded in paraffin, and stained with hematoxylin and eosin as described in Methods and Materials (C). Colons were serially sectioned and degree of dysplasia 
classified according to the following scale: no dysplasia (0), low grade dysplasia (1), mix of low and high grade dysplasia (2), high grade dysplasia (3) and invasive cancer (4). Examples of no dysplasia (a); low grade dysplasia with pseudo-stratification of the nuclei and nuclear enlargement (b); cribriforming tumor glands significant for high grade dysplasia (c); and surface epithelium with higher grade tumor underneath significant for invasion (d) are shown. Magnification: 20X (D). Error bars $=$ SD. $n=3$ per group. Significance: ${ }^{*},{ }^{* \star},{ }^{* \star *}$ denote $p<0.05,0.01,0.001$, respectively. 
A

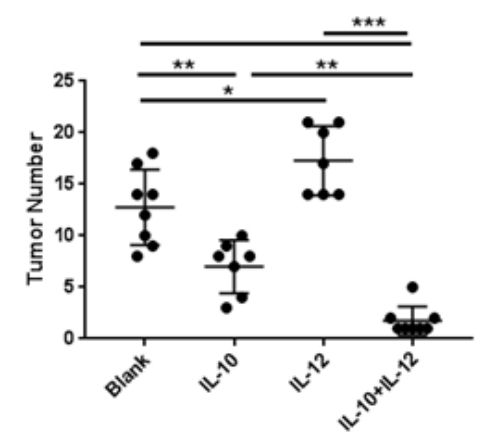

C

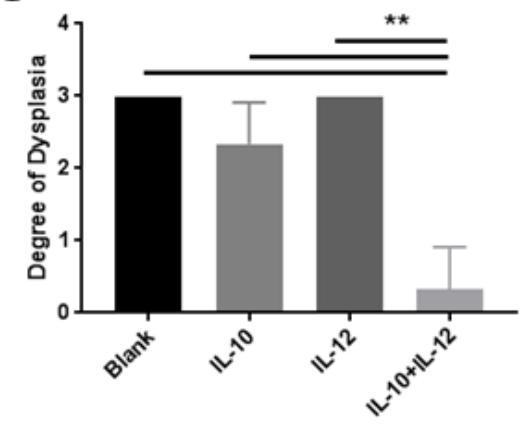

B

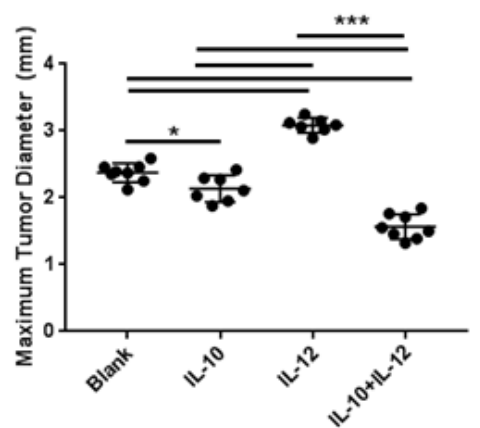

D

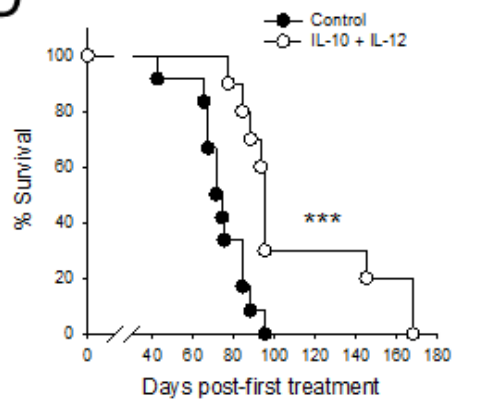

Figure 4: Oral IL-10 and IL-12 act synergistically to eradicate established disease and improve overall survival. (A, B) Colon tumor number and maximum diameter. APC ${ }^{\mathrm{min} /+} /$ $B$ fragilis mice were treated with oral particle-based therapy (blank, IL-10, IL-12, or a mixture of IL-10 and IL-12 particles) as in Figure 1. Mice were then euthanized, and tumor number (A) and maximum tumor diameter $(B)$ in the mouse colon were assessed. Error bars $=S D, n=7-8$ per group. (C) Histologic severity of disease. At the time of euthanasia, colons were fixed and H\&E-stained sections were analyzed as in Figure 1. Error bars $=\mathrm{SD}, \mathrm{n}=3$ per group. (D) Overall survival. APC $\mathrm{min} /+^{\prime} / B$ fragilis mice were treated until euthanasia. $\mathrm{n}=12$ and 10 for control and experimental groups, respectively. Significance: ${ }^{*},{ }^{* *},{ }^{* * *}$ denote $p<0.05,0.01,0.001$, respectively. 
A

TH17 cells

CD8+ T-cells
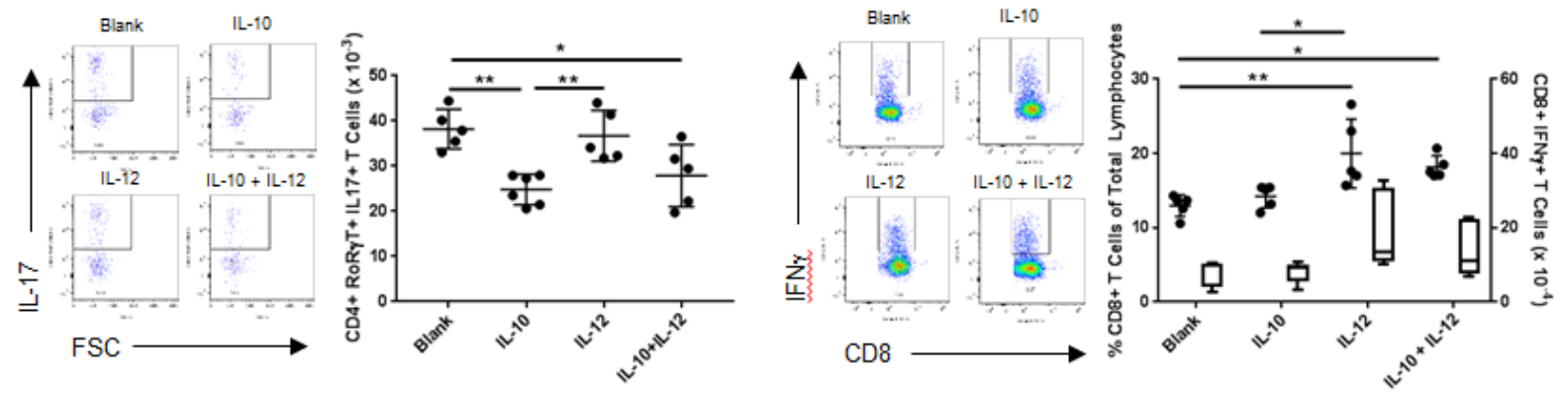

B

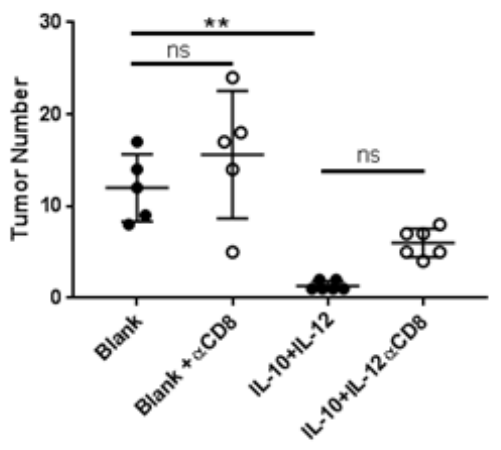

Figure 5: Distinct immunological effects of IL-10 and IL-12 on T-cell subsets are partially responsible for the antitumor synergy. (A) Effect of orally administered IL-10 and IL-12 on Th17 and CD8+ T cells. APC ${ }^{\mathrm{min} /+} / \mathrm{B}$ fragilis mice were treated with oral particlebased therapy (blank, IL-10, IL-12, or a mixture of IL-10 and IL-12 particles) as in Figure 1. Mice were euthanized and lymphocytes were isolated from mesenteric lymph nodes. CD45+CD4+RORyt+ cells were gated on and analyzed for IL-17 production (Th17), and CD45+CD8+ T cells were analyzed for IFNy production (CD8+ T-cells) by FACS. Cell 
numbers shown are per MLN. For CD8+ T-cell panel: filled-in circles $=\%$ of total lymphocytes; box plot $=$ number of $\mathrm{CD} 8+\mathrm{IFN} \gamma+$ cells. Boxes have lines at the median plus lower and upper quartiles, with whiskers extending to show the remaining data. Error bars $=S D, n=5$ per group. (B) Effect of CD8+ $T$ cell depletion on tumor burden. Tumor-bearing $\mathrm{APC}^{\mathrm{min} / \mathrm{+}}$ mice were treated as in Figure 2 in the absence or presence of anti-CD8 $\alpha$ monoclonal antibody administration and assessed for tumor burden. Error bars $=S D, n=5-6$ per group. Significance: ${ }^{*},{ }^{* *}$ denote $p<0.05,0.01$, respectively. 
A

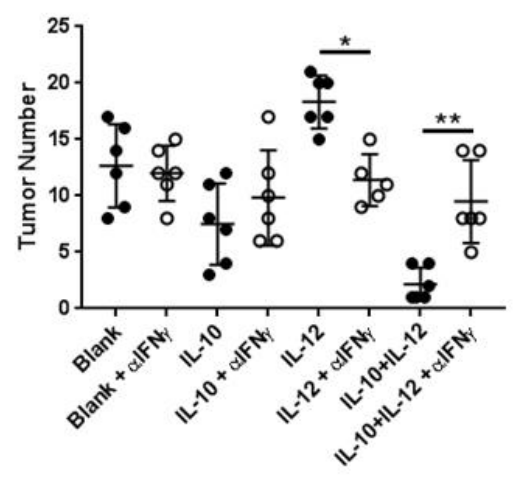

B

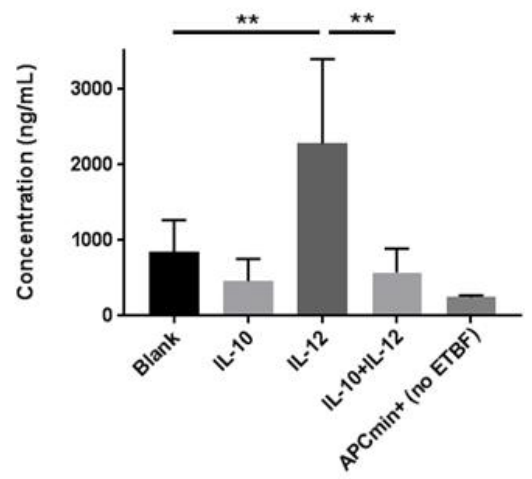

C

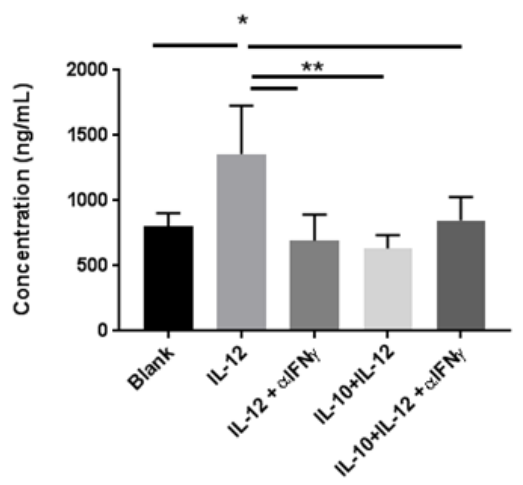

Figure 6: Therapeutic synergy requires IFNy and is in part associated with the effects of cytokines on gut epithelial barrier integrity. (A) Effect of IFNy neutralization on therapeutic outcome. Tumor-bearing APCmin/+ mice were treated as in Figure 2 in the absence or presence of anti-IFNy monoclonal antibody administration and assessed for tumor burden. (B) Gut permeability. Experimental mice were administered FITC-labeled dextran via oral gavage at the end of treatment and sera were analyzed for fluorescence to assess leakage as described in Methods and Materials. (C) Effect of IFNY neutralization on gut permeability. Mice were treated in the absence or presence of IFNy-neutralizing antibody and sera were analyzed as above. Naïve APCmin/+ mice 
served as a control for baseline permeability. Error bars $=S D, n=5-6$ per group for all studies. Significance: ${ }^{*}{ }^{* *},{ }^{* * *}$ denote $\mathrm{p}<0.05,0.01,0.001$, respectively. 
A
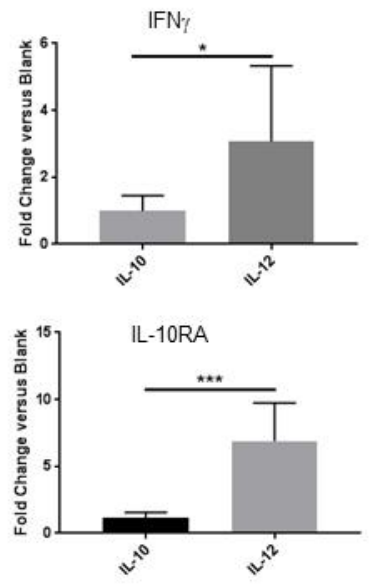

B
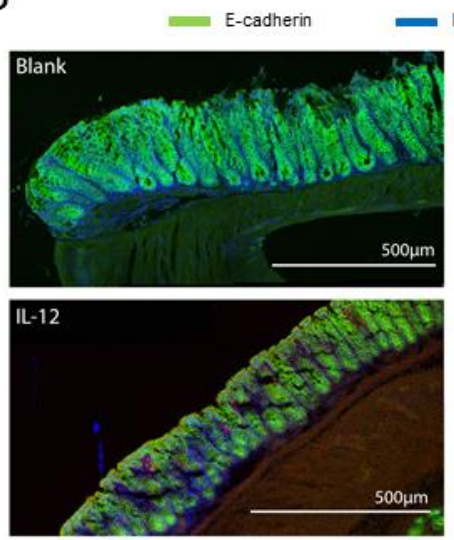

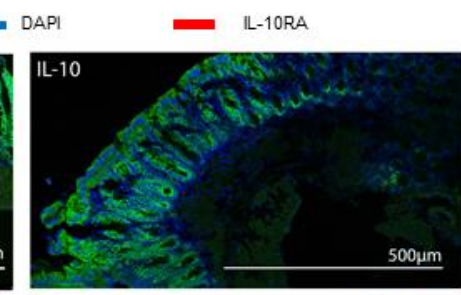

IL-10+ IL-12

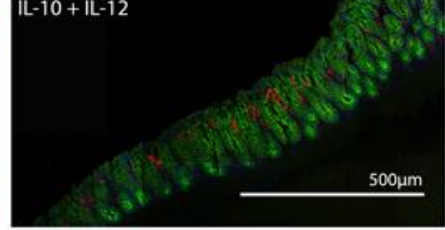

C

IL-10RAKO

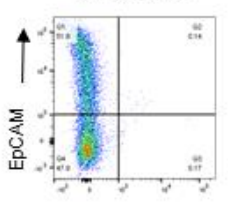

IL-10RA
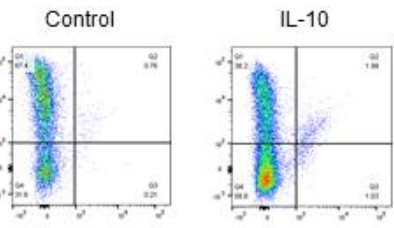

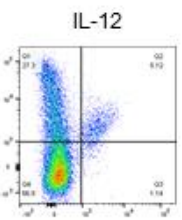

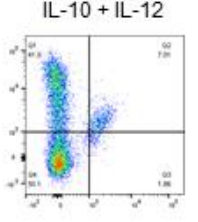

$\mathrm{IL}-10+\mathrm{IL}-12$

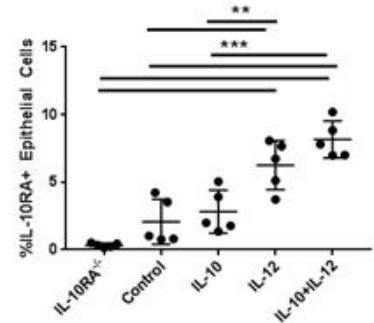

Figure 7: IL-12 induces IFNy and IL-10RA expression in the colon. (A) Quantitative PCR analysis of IL-10RA and IFNy mRNA. Quantitative PCR was performed to evaluate relative changes in IL-10RA and IFNy transcript levels in the colon in blank or cytokine particle-treated mice. (B) IL-10RA expression on colon epithelium. Colon sections from control (blank), IL-10, IL-12 and IL-10+IL-12-treated mice were stained for DAPI (blue), E-cadherin (green), and IL-10RA (red) and visualized by laser-scanning confocal microscopy. (C) FACS analysis of epithelial cell IL-10RA expression. Single cell preparations from colon epithelia of control and treated mice (along with a negative control, i.e. IL-10RA knockout wild-type B6 mice) were stained for EpCAM and IL-10RA expression and were analyzed by flow cytometry. Representative panels and 
quantitative data are shown. Each circle indicates an individual mouse. Error bars = SD. $\mathrm{n}=5$ per group. Significance: ${ }^{*},{ }^{* *},{ }^{* * *}$ denote $\mathrm{p}<0.05,0.01$ and 0.001 , respectively. 
A
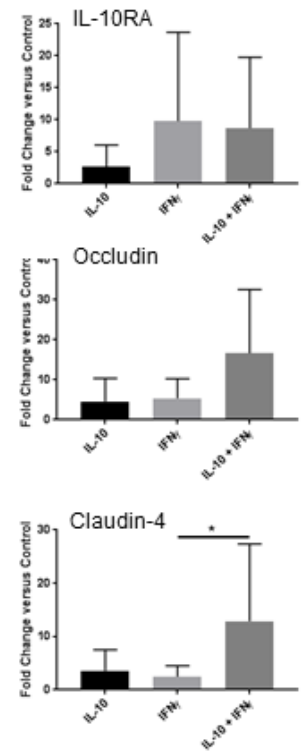

B
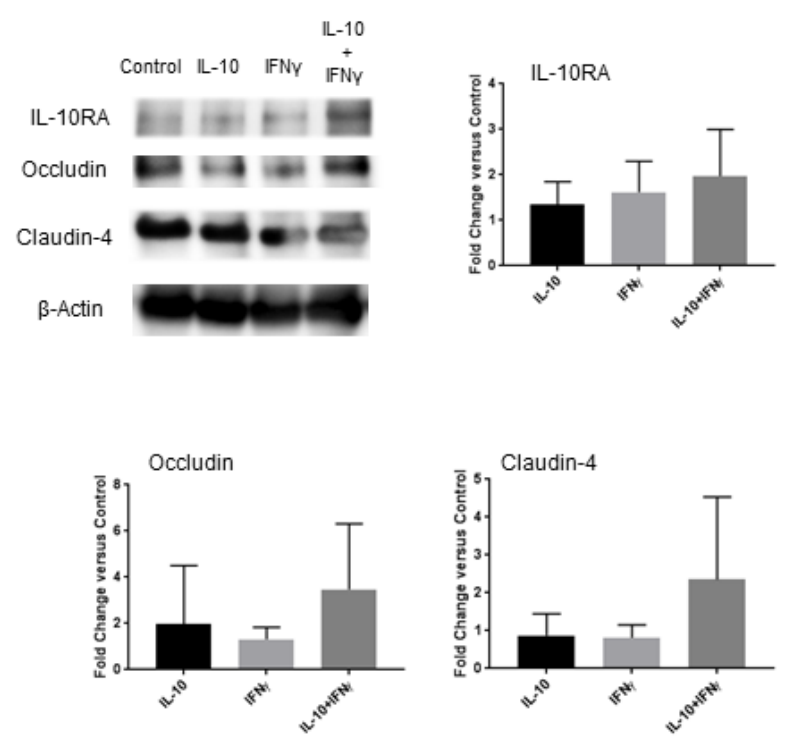

Figure 8: IFNy and IL-10 jointly promote tight junction protein expression in the colon. (A) Quantitative PCR analysis of IL-10RA, occludin and claudin-4 transcripts. Colon explants were cultured for 24 hours in high glucose medium in the presence of recombinant IL-10, IFNy, or both as described in Materials and Methods. RNA was extracted and IL-10RA, occludin and claudin-4 mRNA levels were quantified by qPCR. Error bars $=\mathrm{SD}, \mathrm{n}=6$ per group. (B) Analysis of protein levels. Protein was extracted from colon explants cultured as above and analyzed by Western blotting to detect and quantify IL-10RA, occludin and claudin-4. Representative blot displaying the bands for each protein and $\beta$-actin (loading control) is shown. Signal intensity of each band was normalized to $\beta$ actin for loading in each lane and fold-change was calculated with respect to untreated (control) explants. Combined data from two different blots are shown. Error bars $=S D, n=5-6$ per group. Significance: * denotes $p<0.05$. 


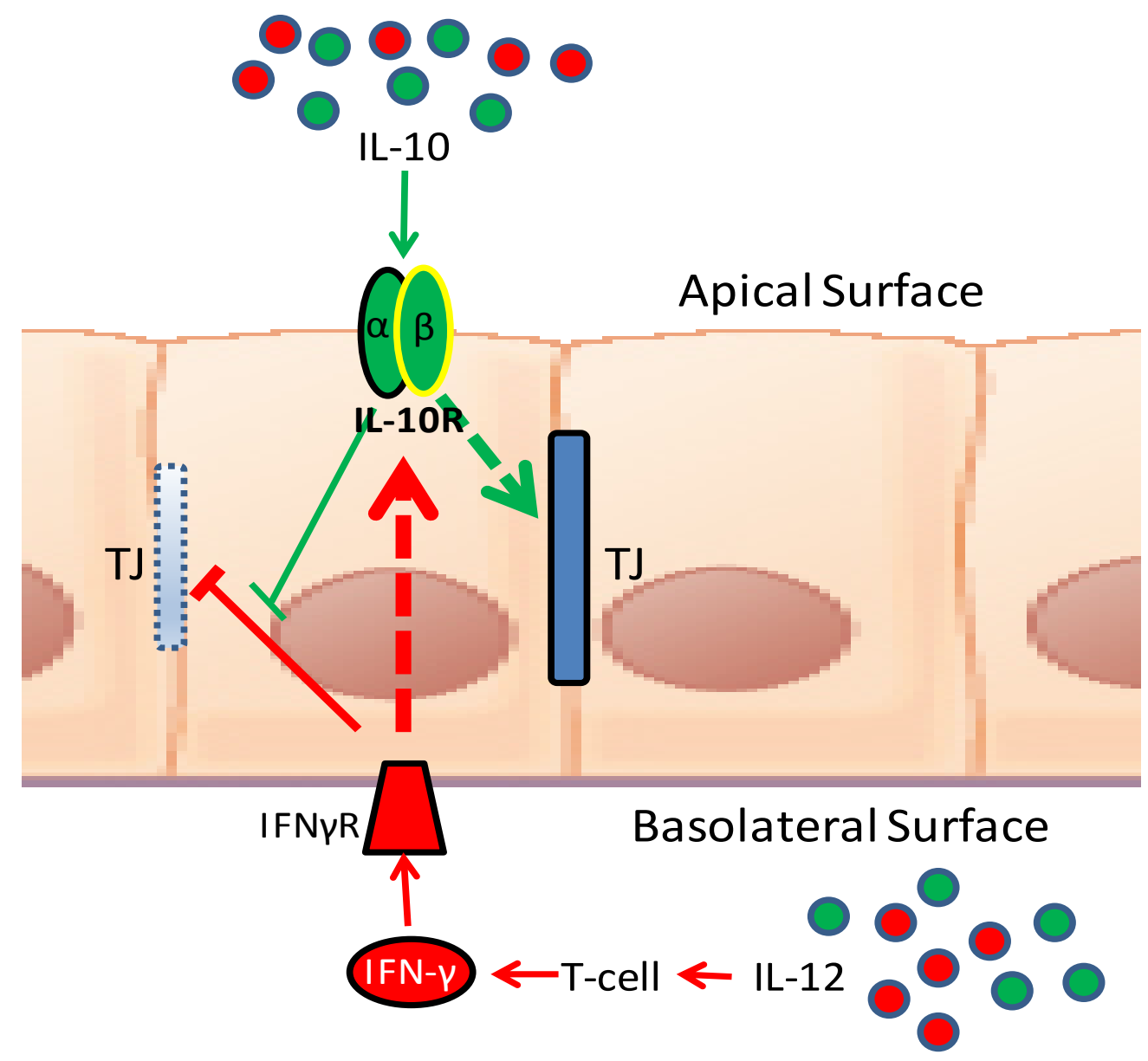

Figure 9: Hypothesized mechanism of action of IL-10 and IL-12 on colon epithelial barrier integrity. IFN-y produced by IL-12 activated T cells binds to IFNyR on the basolateral surface of colon epithelial cells. Signaling through IFNyR results in weakening of tight junctions and epithelial barrier integrity as well as increased expression of IL-10R on the apical surface of colon epithelial cells. IL-10 binding to IL$10 \mathrm{R}$ results in restoration of tight junctions and epithelial barrier integrity. 


\section{CHAPTER FOUR \\ SAVING CHECKPOINT INHIBITORS \\ IDENTIFYING MECHANISMS OF RESISTANCE TO IMMUNE CHECKPOINT INHIBITOR THERAPY IN COLORECTAL CANCER}

\section{Introduction}

Over the last several years, the emergence of immune checkpoint inhibitors (ICls), particularly those targeted against PD-1/PD-L1, has heralded a significant shift in clinical oncology. ${ }^{1-5}$ The therapeutic efficacy of these agents in melanoma and other solid tumors (including lung, kidney, and head and neck cancer) has underscored the powerful anti-tumor capacity of the immune system. ${ }^{6-11}$

Despite the success of anti-PD1 in the aforementioned malignancies, one notable area in which such therapy has failed is in colon cancer. ${ }^{11,12}$ With the exception of tumors that demonstrate significant microsatellite instability and a concomitant defect in mismatch repair machinery, response rates to anti-PD1/PD-L1 therapy in colon cancer have ranged from $0-5 \% .{ }^{13,14}$ Currently, the mechanisms of resistance to anti-PD1 therapy in such patients remain unclear. Given that patients in the latter category comprise approximately $85-90 \%$ of all patients with colon cancer, understanding pathways facilitating resistance and identifying potential therapeutic targets represents a significant area of need in clinical oncology. ${ }^{15,16}$ 
To date, the majority of studies evaluating mechanisms of resistance to anti-PD1/PD-L1 therapy have focused on melanoma given the significant role of such ICls in treatment of this disease. Various groups have demonstrated in both preclinical models and patient samples the roles of differential gene transcription among different tumor foci, Jak1/2 mutations, loss of $\beta 2$-microglobulin, and a number of other innate elements comprising cytokines, chemokines, and proteins involved in angiogenesis, among other things. ${ }^{17-21}$ While these mechanisms of resistance are postulated to extend to other tumor types, which, if any, actually do apply in the setting of other malignancies and whether or not additional mechanisms may play critical roles in mediating resistance to ICls remains unclear.

Based on our findings demonstrating the importance of T17 cells to the persistence and progression of colon tumors (see Chapter 3), we believed that IL-17 producing cells could play a critical role in mediating resistance to anti-PD1 therapy in colon cancer. In the present study, we sought to identify putative immunological mechanisms involved in failure of oral anti-PD1 therapy in a preclinical model of colon cancer. The results presented herein demonstrate the importance of IL-17 and $y \delta$ T cells in colon cancer's resistance to $\mathrm{ICl}$ therapy, thereby pointing toward possible combination therapy strategies to improve ICl efficacy in treatment of colon cancer.

\section{Materials and Methods}

Mice and the tumor model. C57BL/6 (B6) and C57BL/6J-Apc ${ }^{\mathrm{Min}} / \mathrm{J}\left(\mathrm{APC}{ }^{\mathrm{Min} /+}\right)$ mice were purchased from Jackson Laboratory. Enterotoxic B fragilis strain 86-5443-2-2 was a kind gift from Dr. Cynthia L Sears (Johns Hopkins University School of Medicine, Baltimore, Maryland). For colonization with $B$ fragilis, 5-6 week old $\mathrm{APC}^{\mathrm{Min}+}$ mice were administered 
clindamycin $(0.1 \mathrm{~g} / \mathrm{L})$ and streptomycin $(5 \mathrm{~g} / \mathrm{L})$ ad libitum in drinking water for 5 days before oral gavage $\left(\sim 5 \times 10^{7}\right.$ bacteria in PBS) as previously described. All studies were conducted in accordance with guidelines set forth by the Institutional Animal Care and Use Committee at the University of Louisville (Louisville, KY).

Reagents and treatments. Two particle formulations were produced using a modified Phase Inversion Nanoencapsulation (PIN) process: (i) control (no cytokine) and (ii) murine anti-PD1 (Clone J43, BioXCell) with a loading of $1.0 \mu \mathrm{g}$ antibody/mg of particles. Particles were administered via oral gavage $(2.5 \mathrm{mg}$ particles in $0.2 \mathrm{~mL}$ sterile water for blank and anti-PD1 treatments) starting 4 weeks after $B$ fragilis inoculation three times per week for 3 weeks.

Gross intestinal preparation and tumor quantification. Colons were opened longitudinally before being fixed in $10 \%$ neutral buffered formalin. Tumor burden was quantified using a dissecting microscope.

Histology. Formalin-fixed, paraffin-embedded tissue from the distal colon was sectioned

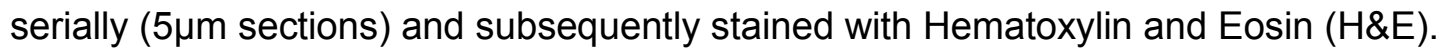
Colon histology was assessed in a blinded fashion by a single tumor pathologist. Each section was classified as harboring no dysplasia, low grade dysplasia, or high grade dysplasia. Colons were then scored according to the following system based on the average severity of dysplasia in the distal colon: 0 - no dysplasia; 1 - low grade dysplasia only; 2 - mixture of low grade and high grade dysplasia; 3 - high grade dysplasia only; 4 - invasive adenocarcinoma. 
Flow cytometry. Membrane and intracellular staining of MLN cells and lamina propria lymphocytes (LPL) were performed as described. ${ }^{22}$ The following antibodies were used: CD4 (GK1.5, BioLegend), CD8a (53-6.7, BD Biosciences), CD45.2 (104, BD Biosciences), CD16/CD32 (93, BioLegend), үסTCR (GL3, BioLegend), IL-17A (TC1118H10.1, BioLegend), IFNy (XMG1.2, BD Biosciences).

Lymphocyte depletion and functional blockade studies. Anti-mouse CD4 (GK1.5, BioXCell) was given intraperitoneally (ip) to $\mathrm{APC}^{\mathrm{min} /+}$ mice $(0.2 \mathrm{mg}$, three times per week for 3 weeks) to deplete CD4+ T lymphocytes. Anti-mouse $ү / \delta$ TCR (UC7-13D5, Leinco Technologies) was given ip to APC ${ }^{\mathrm{min} /+}$ mice $(0.25 \mathrm{mg}$, twice per week for 3 weeks) to deplete $ү / \delta$ TCR+ T lymphocytes. Anti-mouse IL17 (17F3, BioXCell) was injected ip $(0.2 \mathrm{mg}$, three times per week for 3 weeks). All treatments were initiated on treatment day -1 (the day before receiving their first oral immunotherapy treatment) and again on treatment day 0 (the day of their first oral immunotherapy treatment).

Statistical Analysis. Two-tailed student's t-test was used to determine the significance of the differences between control and experimental groups in pairwise comparisons. In experiments with multiple groups homogeneity of inter-group variance was analyzed by one-way ANOVA with Tukey's honest standard difference for multiple comparisons. $P$ values less than 0.05 were considered statistically significant. Statistical analyses were performed using GraphPad Prism 7 (GraphPad Software, La Jolla, California, USA) and MedCalc version 17.9.7 (MedCalc Software, Ostend, Belgium). 


\section{Results}

Orally administered checkpoint inhibitor monotherapy is ineffective against colon cancer

Outside of cases with high microsatellite instability, previous work has demonstrated a general lack of efficacy of immunotherapy in colon cancer. To evaluate whether orally administered anti-PD1, which would augment local delivery of checkpoint inhibitor to the colon, would result in effective treatment, mice were treated with either blank or antiPD1-containing particles by oral gavage for three weeks. No significant differences were noted in tumor number, maximum tumor diameter, or histologic severity of disease (Figures 1A, 1B). These findings confirmed failure of anti-PD1 monotherapy in treatment of colon cancer, suggesting that simply altering the method of delivery could not overcome therapeutic resistance.

IL-17 blockade significantly improves the efficacy of anti-PD1 therapy in treatment of colon cancer.

IL-17 has been demonstrated to play a significant role in the development and progression of colon cancer. Given the known tumor-promoting role of IL-17 in this setting, we hypothesized that blockade of this signaling could result in a more favorable immune microenvironment that could augment the efficacy of anti-PD1 treatment. Towards this end, mice were subjected to IL-17 blockade and treated with either blank or either anti-PD1 containing particles for 3 weeks. While IL-17 blockade significant improved tumor number, compared to treatment with either blank or anti-PD1 particles alone, the combination of IL-17 blockade and anti-PD1 treatment resulted not only in near complete eradication of tumors, but also significant reductions in both maximum 
tumor diameter and histologic severity (Figure 2A-C). These findings indicate that IL-17 plays a critical role in mediating failure of anti-PD1 therapy in colon cancer.

$\Gamma \delta T C R+T$ cells, but not CD4+ T cells, play a critical role in IL-17 mediated resistance to anti-PD1 therapy

As previously mentioned, several cell populations are known to produce IL-17. However, as previously demonstrated, two subsets of T cells - үס17 cells and CD4+ Th17 cells - play a critical role in promoting colon tumor development. To determine which of these cell populations contributed to IL-17 production involved in anti-PD1 resistance, mice were subjected to either CD4+ T cell depletion or $ү \delta$ TCR depletion in the context of treatment with either blank or anti-PD1 particles. CD4+ T cell depletion had no significant effect on either tumor number of maximum tumor diameter, either alone or when combined with anti-PD1 therapy (Figure 4A, 4B). However, depletion of Yס TCR + T cells resulted in a significant reduction in tumor number but not maximum tumor diameter (Figure 3A, 4B). These results suggest that $ү \delta \mathrm{T}$ cells may be major mediators of anti-PD1 resistance in colon cancer in an IL-17 dependent manner.

\section{Discussion}

Clinical trials of ICI therapy, particularly with anti-PD1/PD-L1, have demonstrated minimal, if any, effect in patients with microsatellite stable colon cancer. Despite several mechanisms of resistance having been postulated, none have been conclusively shown in either preclinical models or human subjects. Our results indicate that IL-17 represents a critical element mediating anti-PD1/PD-L1 resistance in microsatellite stable colon 
cancer. Moreover, a large component of this IL-17 effect appears to derive from $ү \delta ~ T$ cells in the tumor microenvironment.

The tumor-promoting effects of IL-17 in colon cancer have been demonstrated both in preclinical models and IL-17's role in colon cancer. IL-17 binds to its ubiquitouslyexpressed cognate receptor (IL17RA) to result in release of pro-inflammatory cytokines and chemokines as well as increased expression of angiogenesis promoting factors (i.e. VEGF, prostaglandin E1, prostaglandin E2). Indeed, IL-17 has been shown to promote tumor growth and augment vascularity in murine models of colon cancer. ${ }^{23}$ Human studies have also demonstrated an association of IL-17 mRNA with increasingly dysplastic colon tumors along the adenoma to carcinoma pathway as well as increased levels of IL-17 in colon cancer tissue, with the IL-17E subtype related to tumor cell differentiation. $^{24,25}$

In colon cancer, two cell subsets have been strongly linked with IL-17 production: Th17 cells and $ү \delta T 17$ cells. The role of Th17 cells in promoting colon tumorigenesis has been previously demonstrated. ${ }^{26,27}$ However, recent evidence has suggested that IL-17 producing $ү \delta ~ T$ cells play a critical role in tumor progression. These cells, largely expanded in the tumor microenvironment, have been shown to accumulate in colon tumor tissue, potentially being polarized therein by cytokines (including dendritic cellproduced IL-23) present in the tumor microenvironment. ${ }^{28}$ This situation results from weakened colothelial barrier integrity, bacterial translocation, and resultant attraction of dendritic cells into peritumoral tissue. ${ }^{28}$ The end result of $ү \delta T 17$ activation and proliferation is recruitment of PMN-type myeloid derived suppressor cells and 
subsequent immunosuppression. ${ }^{28}$ Taken together with our findings, these data underscore the importance of $\mathrm{y} \delta \mathrm{T} 17$ cells in supporting a microenvironment favoring tumor growth in colon cancer.

In the context of previous work, our findings suggest that the relative importance of Th17 and $y \delta \mathrm{T} 17$ to promoting tumor development and growth may exhibit temporal variation. As previously mentioned, Th17 cells have been shown to be important in colon tumorigenesis, findings supported by work from our group demonstrating the abrogation of tumor formation with CD4+ T cell depletion. ${ }^{22,26,27,29}$ In the setting of established colon tumors, however, our data suggest that $ү \delta \mathrm{TT} 17$ cells appear to play a more critical role in maintaining a tumor-promoting, immunosuppressive microenvironment. While future work is required to elucidate the mechanisms underpinning this observation and appropriately test the hypothesis of a time-dependent importance of Th17 and yסT17 cells in colon tumorigenesis and progression, the role of $ү \delta \mathrm{T} 17$ cells later in disease may stem from the changes in colon permeability, bacterial translocation, and DC recruitment associated with increasingly dysplastic lesions.

Our findings have exciting implications for future applications in treatment of patients with colon cancer. Both IL-17 and $y \delta$ T cells represent putative targets for therapies that could be combined with anti-PD1/PD-L1 to augment the efficacy of the latter agent. While no anti-y $\delta$ T cell antibody is currently approved for clinical use, an FDA-approved anti-IL-17 antibody, secukinumab (Cosentyx; Novartis International AG, Basel, Switzerland), is currently being used in treatment of patients with plaque psoriasis. With a low side-effect profile (approximately 10\% adverse event rate), combination of this 
agent with anti-PD1/PD-L1 represents an enticing possibility for future clinical trials in

patients with colon cancer. ${ }^{30}$ If successful, such combination therapy would represent a significant advance in the treatment of colon cancer, providing patients and physicians with a presumably well-tolerated, effective treatment regimen with application in a wide variety of disease settings.

\section{Conclusions}

The results of these studies suggest that IL-17-producing $ү \delta$ T cells play a critical role in mediating resistance of colon tumors to anti-PD1 therapy. While the detailed mechanisms underlying this observation remain areas for future study, the phenomena described herein represent exciting possibilities for clinical trials combining existing antiIL-17 therapy with anti-PD-1 therapy in patients with microsatellite stable colon cancer, anti-PD1 refractory microsatellite unstable colon cancer, and, potentially, other tumor types with in which IL-17 plays a role in tumor development. 


\section{Figures}

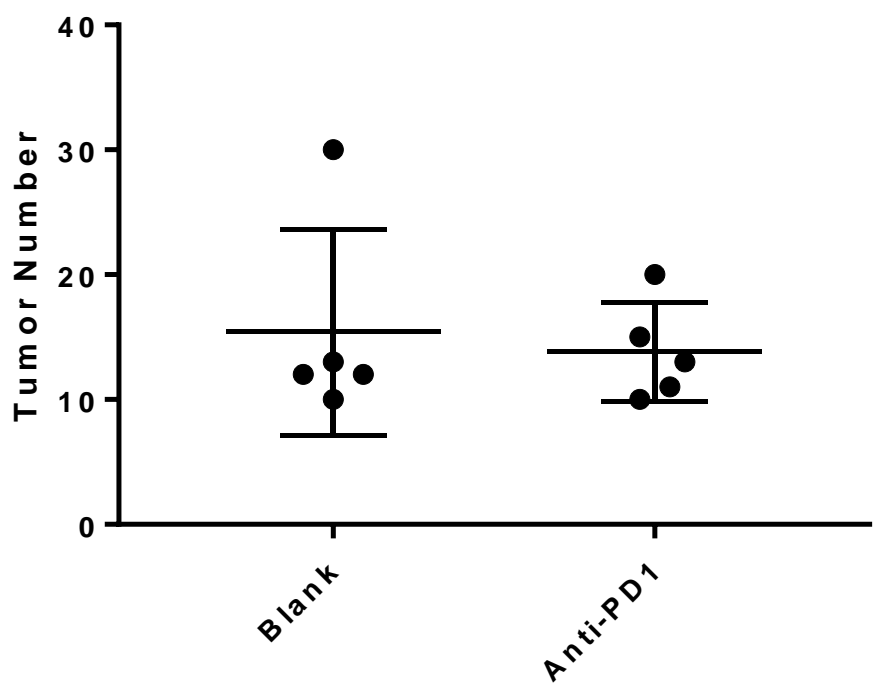

A.

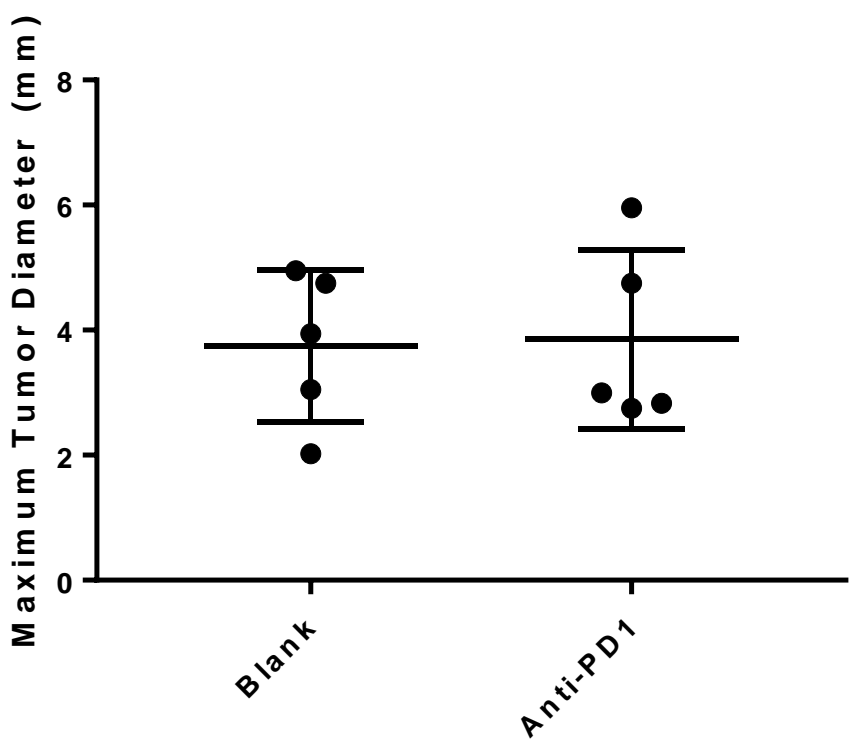

B.

Figure 1: Oral anti-PD1 monotherapy is ineffective in treatment of established colon tumors. $(A, B)$ Colon tumor number and maximum diameter. $A P C^{\mathrm{min} /+}$ mice were treated with oral particle-based therapy (either blank particles or particles loaded with murine anti-PD1 antibody) for 3 weeks beginning 4 weeks after enterotoxic $B$ fragilis inoculation. 
Mice were then euthanized, and tumor number $(A)$ and maximum tumor diameter $(B)$ in the mouse colon were assessed. Error bars $=S D, n=5$ per group. 


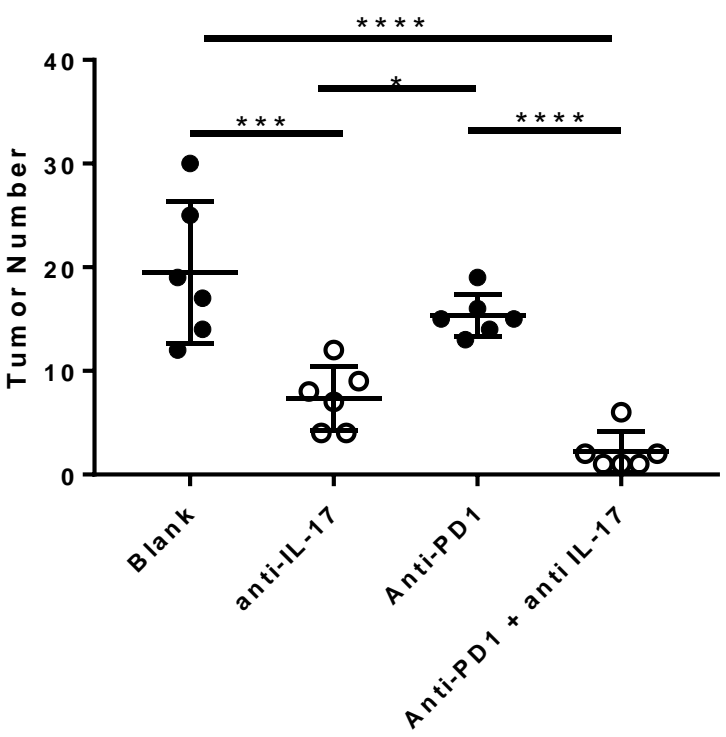

A.

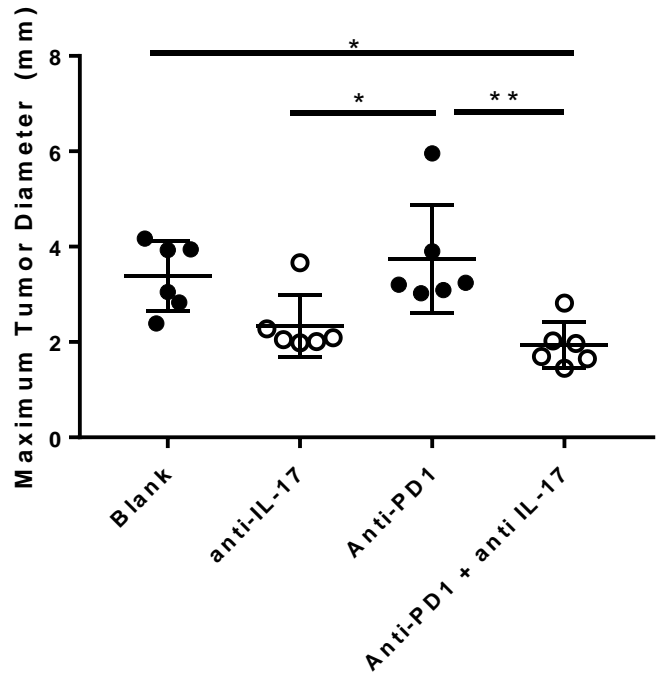

B. 


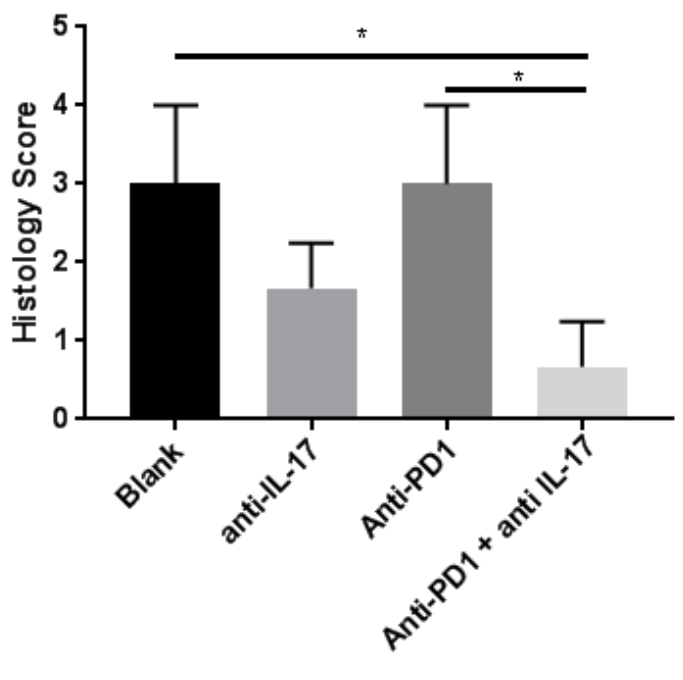

Figure 2: IL-17 blockade significantly improves the efficacy of oral anti-PD1 in treatment of established colon tumors. (A, B) Colon tumor number and maximum diameter. $\mathrm{APC}^{\mathrm{min} /+}$ / B fragilis mice were treated with oral particle-based therapy (blank, anti-PD1) for 3 weeks with and without IL-17 blockade by intraperitoneal injection of anti-IL-17A monoclonal antibody. Mice were then euthanized, and tumor number $(A)$ and maximum tumor diameter $(B)$ in the mouse colon were assessed. Error bars $=S D, n=6$ per group. 
A.

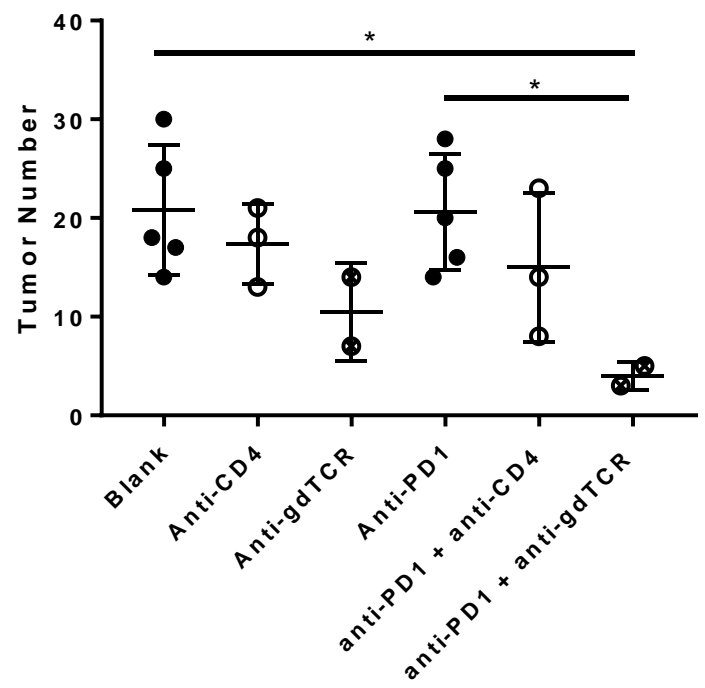

B.

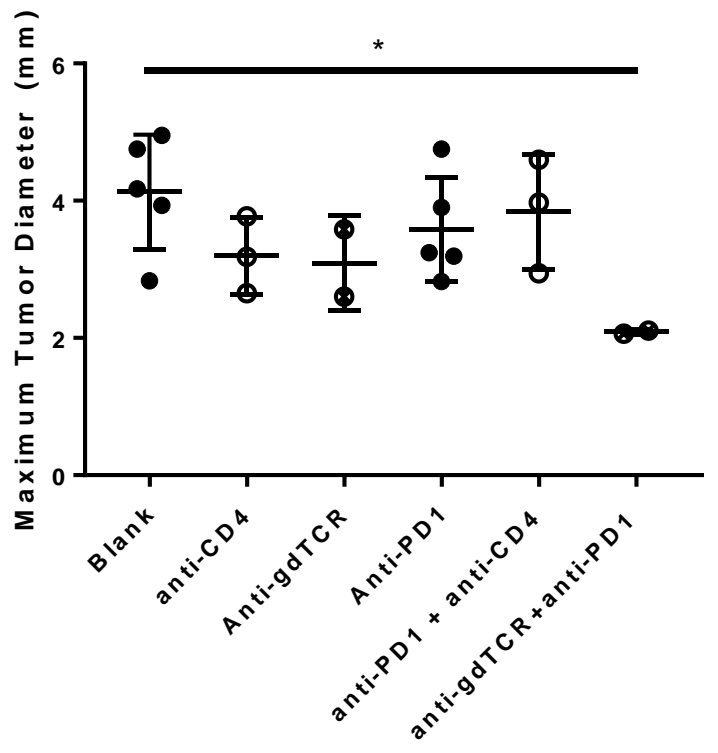

Figure 3: IL-17-based resistance to anti-PD1 therapy appears mediated by үסTCR+ T cells but not CD4+ T cells. (A, B) APC ${ }^{\mathrm{min} /+} / \mathrm{B}$ fragilis mice were treated with oral particle-based therapy (blank, anti-PD1) for 3 weeks with and without either CD4+ T cell depletion or $ү \delta$ T cell depletion by intraperitoneal injection of $\alpha-C D 4$ or $\alpha-\gamma \delta$ TCR monoclonal antibody. Mice were then euthanized, and tumor number $(A)$ and maximum tumor diameter $(B)$ in the mouse colon were assessed. Error bars $=S D, n=2-5$ per group. 


\section{CHAPTER FIVE}

\section{THE NEXT FRONTIER}

ESTABLISHING A PRACTICAL MODEL FOR SPONTANEOUS COLON TUMORS

\section{Introduction}

Among patients with colorectal cancer, approximately $10-25 \%$ initially present with liver metastases. ${ }^{1}$ Moreover, even among patient who undergo successful resection of their primary tumor, nearly $50 \%$ will ultimately develop liver metastases. ${ }^{1}$ While a solitary liver metastasis or oligometastatic disease often responds well to systemic chemotherapy and resection, the metastatic disease setting still represents the area in which novel therapies are most sorely needed, particularly since chemotherapy converts patients to resectability in $7-50 \%$ of cases. $^{2}$ Thus, a practical, high-fidelity preclinical model for colorectal liver metastases remains essential for the effective evaluation of new therapeutic agents.

Currently, numerous models of colon cancer exist, ranging from those involving chemical induction (1,2-dimethyhydrazine $(\mathrm{DMH})$ and azoxymethane $(\mathrm{AOM}))$ to a combination of a germline mutation and bacterially-induced inflammation (APC Min/enterotoxic Bacteroides fragilis) to genetically modified mice that allow for sitespecific induction of mutations commonly found in patients with colon cancer (e.g. Apc, Kras, Msh2). ${ }^{3-6}$ While each has its own advantages and disadvantages, they all provide reasonable model systems for assessing the development and progression of both 
sporadically arising colon tumors and those developing in the setting of chronic inflammation (i.e. colitis). However, none of these models produce colon tumors that spontaneously metastasize to the liver, thereby limiting their ability in assessing both the process of metastasis development and the impact of antineoplastic agents on both primary tumors and metastatic disease. $^{7}$

To study colorectal liver metastases, researchers have largely had to rely upon orthotopic injection of tumor cells (i.e. injection directly into the liver), implantation of tumors into colons of nude mice, or injection of tumor cells into the portal vein to allow diffuse seeding of the liver. ${ }^{4,8-10}$ Unfortunately, none of these models accurately reflects the clinical setting in which such disease develops. In order to address the need for a more clinically-relevant model of colorectal liver metastases (and colon cancer in general), KE Hung and colleagues developed a model utilizing site-specific, inducible mutations in $A p c$ and Kras that reliably resulted in formation of one to two colon tumors that progress along the adenoma to carcinoma sequence and progresses to liver metastases in approximately $20 \%$ of mice. $^{7}$

Use of this model, while high-fidelity, has not been reported in any studies since its publication. Several reasons likely explain this observation. First, the model requires a significant amount of technical expertise due to multiple surgical elements (laparotomy, colostomy, and surgical repair of both incisions). Additionally, the procedure is quite inefficient and time intensive, with only $50 \%$ of mice developing carcinomas at 20 weeks after tumor induction and metastases developing in only $20 \%$ of mice 24 weeks after tumor induction. ${ }^{7}$ Thus, the model, while clinically relevant, remains largely impractical for laboratory use. 
Given the well-described use of mouse colonoscopy, we believed that using endoscopic methods would allow us to circumvent several of the technical complexities associated with this model. ${ }^{11,12}$ Additionally, such methods would allow for targeted mutation at a specific site in the distal colon which could be easily and reliably monitored for tumor development and progression. Herein, we describe a minimally-invasive endoscopic methods for induction of $A p c$ and Kras mutations resulting in distal colon adenocarcinoma development in addition to eventual development of gross liver metastases.

\section{Methods}

\section{Animals}

Apc conditional knockout mice (C57BL/6-Apctm1Tyj/J, Jackson Labs) were crossed with mice bearing a latent mutant Kras allele (LSL-K-ras G12D, Jackson Labs). Mice had Apc and Kras mutations, respectively, under control of an LSL system, with mutation inducible by insertion of a Cre recombinase-expressing adenovirus. Breeding consisted of a C57BL/6-Apctm1Tyj/J male crossed with a LSL-K-ras G12D female. Progeny were genotyped using the methods described by Jackson Labs. Progeny positive for both latent $A p c$ and Kras alleles were utilized for experimentation.

\section{Mouse Colonoscopy and Adenovirus Infection of Colon Epithelium}

Mice were anesthetized using ketamine/xylazine anesthesia. After achieving an adequate level of anesthesia an 8F pediatric cystoscope was introduced into the mouse anus and advanced proximally approximately $2-3 \mathrm{~cm}$. Air was insufflated during this time to allow for adequate visualization while avoiding perforation. The colon was then flushed with $300 \mathrm{~mL}$ 1x PBS, and $200 \mathrm{~mL}$ 0.25\% trypsin with EDTA was instilled via the working port of the colonoscopy while slowly withdrawing the instrument. Mice were 
positioned with their head down for 15 minutes to allow for trypsin digest of intercellular adhesion molecules between colon epithelial cells. The colonoscopy was subsequently reinserted and biopsy forceps were used to create a discrete break in the epithelium of the distal colon under direct visualization. Care was taken to avoid taking a fullthickness biopsy and causing a colon perforation. To induce gene mutation at the site of the biopsy, $10^{9} \mathrm{PFU}$ of Ad5CMVcre High Titer (HT) (Viral Vector Core, University of lowa) in $50 \mu \mathrm{L}$ of $1 \times$ PBS were injected into the muscularis mucosa using a microneedle inserted through the colonoscopy working port under direct visualization. Care was taken not to allow transcolonic needle penetration with viral instillation into the peritoneal cavity. After instillation, the needle was withdrawn into the working port, and the colonoscope was withdrawn slowly. Mice were observed in cages placed on a $37^{\circ} \mathrm{C}$ heating pad until they began moving spontaneously.

\section{Monitoring Tumor Development}

To monitor for tumor development and progression, mice underwent serial biweekly colonoscopy as described above beginning two weeks after virus instillation. Adenomas were biopsied using biopsy forceps inserted through the working port of the colonoscope. Biopsies were placed in $10 \%$ neutral buffered formalin for histologic analysis. Mice were then euthanized at two week intervals beginning six weeks after adenoma formation to assess time to adenoma formation and time from adenoma formation to carcinoma development. At time of euthanasia, colons were excised, bisected longitudinally, and rinsed with 1x PBS to remove stool and mucous before being placed in $10 \%$ neutral buffered formalin. Colons were embedded in paraffin and sectioned at $10 \mu \mathrm{m}$ intervals before being stained with hematoxylin and eosin (H\&E) for pathology analysis. 


\section{Assessing for Metastasis Development}

To determine time to metastasis development, mice were euthanized beginning 12 weeks after virus instillation. Colon, mesenteric lymph nodes (MLN), and liver were excised. Colons were treated as described above, and all organs were placed in 10\% neutral buffered formalin. Organs were embedded in paraffin and sectioned at $10 \mu \mathrm{m}$ intervals before being stained with hematoxylin and eosin (H\&E) for pathology analysis.

\section{Histologic Analysis}

H\&E stained slides were reviewed by an experienced colorectal pathologist to determine the presence of adenoma, carcinoma, and metastatic disease (in the lymph nodes and liver). Descriptive analysis was provided in addition to classification of lesions as adenoma or carcinoma.

\section{Results}

Endoscopic adenovirus instillation is feasible and safe As previously mentioned, we initially sought to determine whether we could employ endoscopic methods to improve the feasibility of the model previous described by Hung, et al. ${ }^{7}$ The apparatus and setup used are depicted in Figure 1. For optimal execution, the procedure was noted to require two operators: one for operation of the biopsy forceps and microneedle and one for manipulation of the colonoscope. This system enabled successful creation of a mucosal break and adenovirus injection in 20 of 22 mice (91\%) on five separate occasions. Mice tolerated the procedure well; only two mice developed colon perforations and required euthanasia. 
Endoscopic adenovirus instillation results in adenoma formation 2-3 weeks after mutation induction

To evaluate whether endoscopic adenovirus instillation reliably resulted in tumor formation and the time to initial adenoma formation, mice underwent colonoscopy every two weeks beginning two weeks after adenovirus instillation. Adenoma formation was noted at two weeks after adenovirus injection (Figure 2A). Time to adenoma formation was similar in five mice injected with adenovirus. Histologic analysis of biopsies obtained from the lesions confirmed development of adenomas (Figure 2B).

Histologic analysis demonstrates adenoma to carcinoma transition at 12 weeks after adenovirus injection

To assess the time from adenoma formation to the development of invasive carcinoma, three mice were euthanized at 12 weeks after adenovirus injection (10 weeks after adenoma development). Histologic analysis of the tumors demonstrated invasive carcinoma (Figure 3A). Histologic evaluation of the liver at this time demonstrated no evidence of metastatic tumor deposits (Figure 3B).

Spontaneous development of liver metastases

For evaluation of time to development of lymph node and/or liver metastases, mice were sequentially euthanized beginning at 20 weeks after adenovirus injection. Histologic analysis did not demonstrate any evidence of microscopic disease in either mesenteric lymph nodes or colon until 48 weeks of age. One of three mice euthanized at this time was noted to have gross evidence of widespread liver metastases (Figure 4). 


\section{Discussion}

As previously mentioned, current models of colorectal cancer in general and liver metastases in particular fail to accurately reflect the most prevalent clinical scenario of spontaneous arising colon tumors (adenomatous polyps) that eventually transition to carcinoma and, in certain cases, to colorectal liver metastases. ${ }^{4,13}$ Despite the development of a model that successfully recapitulates this scenario, its technical and logistic impracticality has limited its use. The model described herein overcomes many of those obstacles and represents a significant practical improvement on the aforementioned system.

The method we describe likely works reliably and efficiently for several reasons. Using biopsy forceps to create a discrete mucosal break through which to perform submucosal adenovirus instillation allows for several key elements: 1) a tumor confined to a known, specific region in the distal colon, 2) the ability to administer a high concentration of virus in a small volume to a naturally confined region, avoiding the need to segmental colon occlusion and incubation, and 3) improved adenovirus penetration into cells with transformation potential (i.e. cells other than well-differentiated epithelial cells), maximizing transformation efficiency. Furthermore, by utilizing colonoscopy for instillation, our method not only allows for visual confirmation of successful injection, but also facilitates stool clearance. As previously mentioned, such an approach also spares mice the morbidity of a laparotomy and colostomy and, as a result, decreases the time mice must be under general anesthesia. 
As previously discussed, the value of a high-fidelity model for colon cancer and colorectal liver metastases cannot be overstated. Such a model would enable more granular, detailed analysis of the genetic, molecular, and immunologic elements associated with and responsible for the adenoma to carcinoma transition and the development of liver metastases. Additionally, it would allow for realistic evaluation of the performance of novel imaging modalities (i.e. multispectral optoacoustic tomography (MSOT)) along with tracers and dyes in conjunction with existing modalities (i.e. magnetic resonance imaging (MRI), computed tomography (CT), or high-resolution ultrasound $(\mathrm{U} / \mathrm{S})$ ) in early detection of primary colon tumors and liver metastases. Finally, such a model represents a significant improvement over those currently available for evaluation of the therapeutic potential of novel cytotoxic and immune-based therapies along the spectrum of colorectal cancer.

Despite the findings presented herein, several aspects of this model system require further investigation. The exact time at which mice develop microscopic liver metastases remains unclear, as does the proportion of mice who develop hepatic metastases. Accurately elucidating these elements will likely require a large, longitudinal study employing a combination of colonoscopy and sequential non-invasive liver imaging using high-resolution ultrasound and euthanasia. Furthermore, the degree to which this model reflects the known mutational and methylation profiles of human colon adenomas, carcinomas, and liver metastases remains unknown. Targeted or whole genome analysis of tumor tissue from a large cohort of mice could help delineate the degree to which this model parallels human colon cancer on a genetic and epigenetic level. 


\section{Conclusions}

The results described herein demonstrate that a colonoscopy-based virus delivery to a site of mucosal disruption in mice with inducible $A p c$ and Kras mutations represents a feasible, safe, and effective method of inducing spontaneous colon tumors that demonstrate a stepwise progression along the adenoma-carcinoma-metastasis pathway. Such a model enables evaluation of study of the genetic and immunologic perturbations both locally and systemically throughout this process. Furthermore, it provides an extremely clinically-relevant system for evaluation of the therapeutic efficacy of novel antineoplastic agents as well as imaging modalities for detecting sub-clinical disease. 
Figures

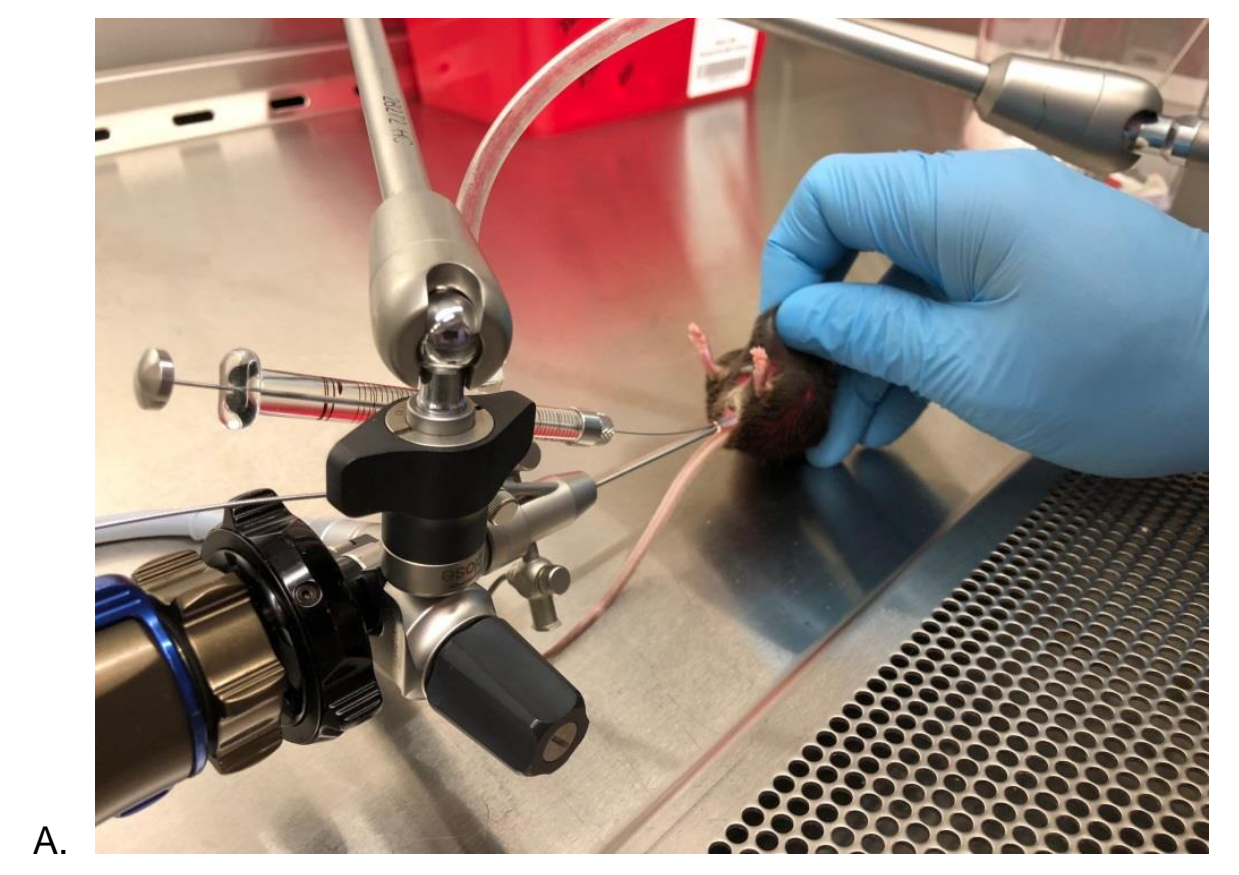

B.

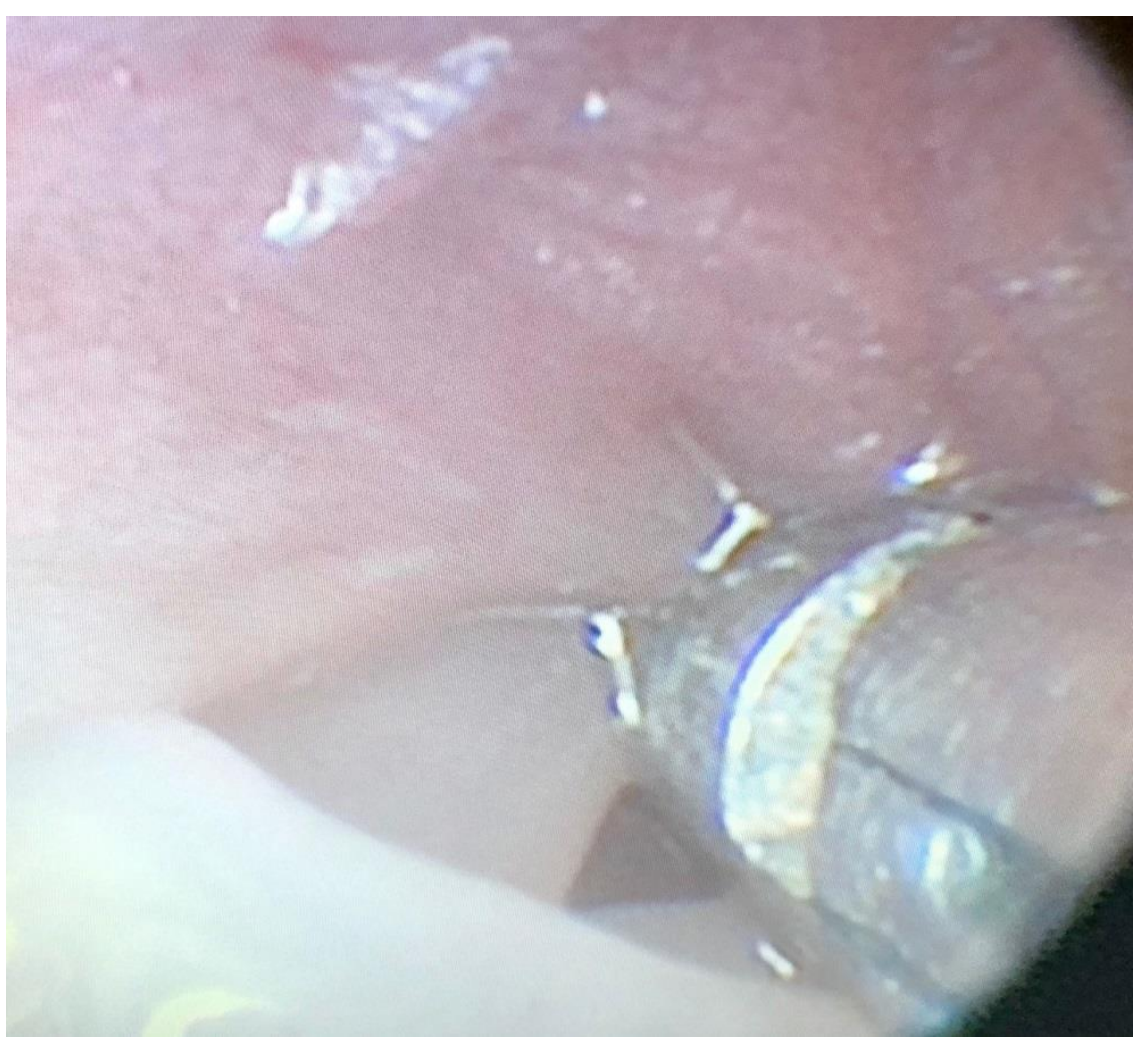



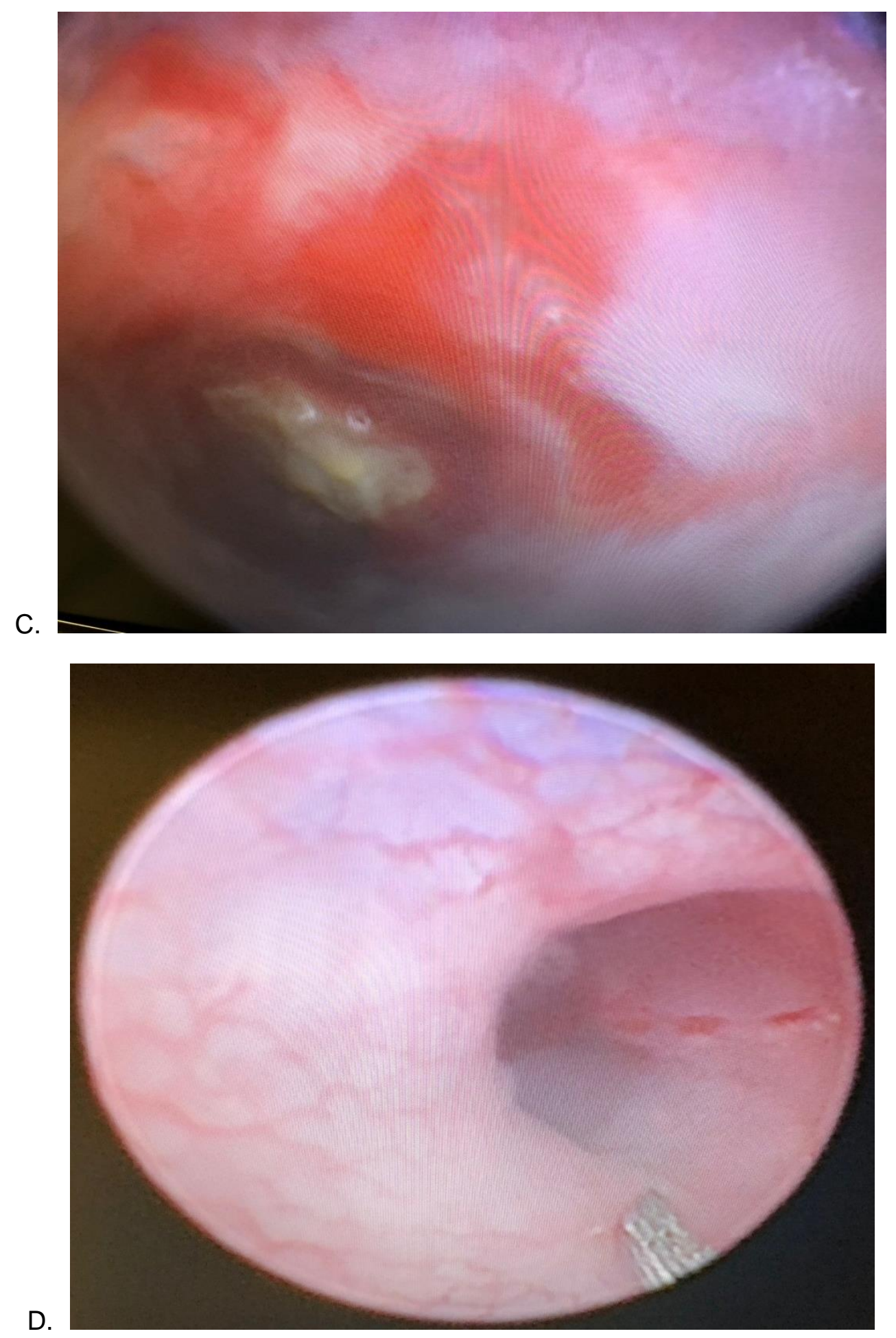

Figure 1: Mouse colonoscopy and adenovirus instillation. (A) Complete setup for mouse colonoscopy, including colonoscope, colonoscope holder, and microneedle. 
(B, C) Biopsy forceps grasping tissue (B) and mucosal break following tissue biopsy

(C). (D) Microneedle inserted into mucosa for adenovirus delivery. 
A.
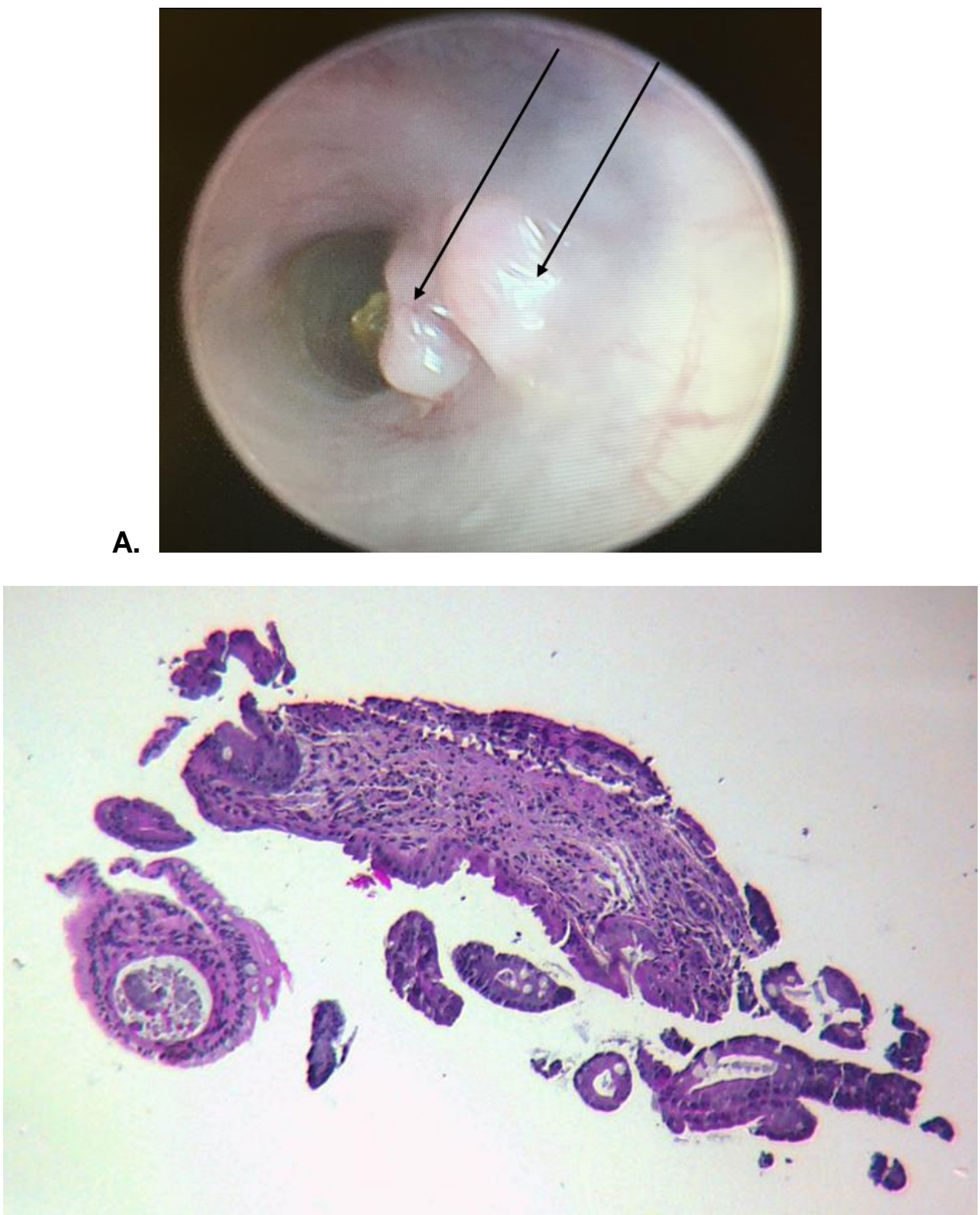

Figure 2: Gross and histologic demonstration of colon adenoma formation. (A) Endoscopic visualization of colon adenoma two weeks after adenovirus instillation. Black arrows indicate polyps. (B) Histology of colon tumor biopsy (4x) demonstrating hyperplasia and mild architectural distortion consistent with a colon adenoma. 


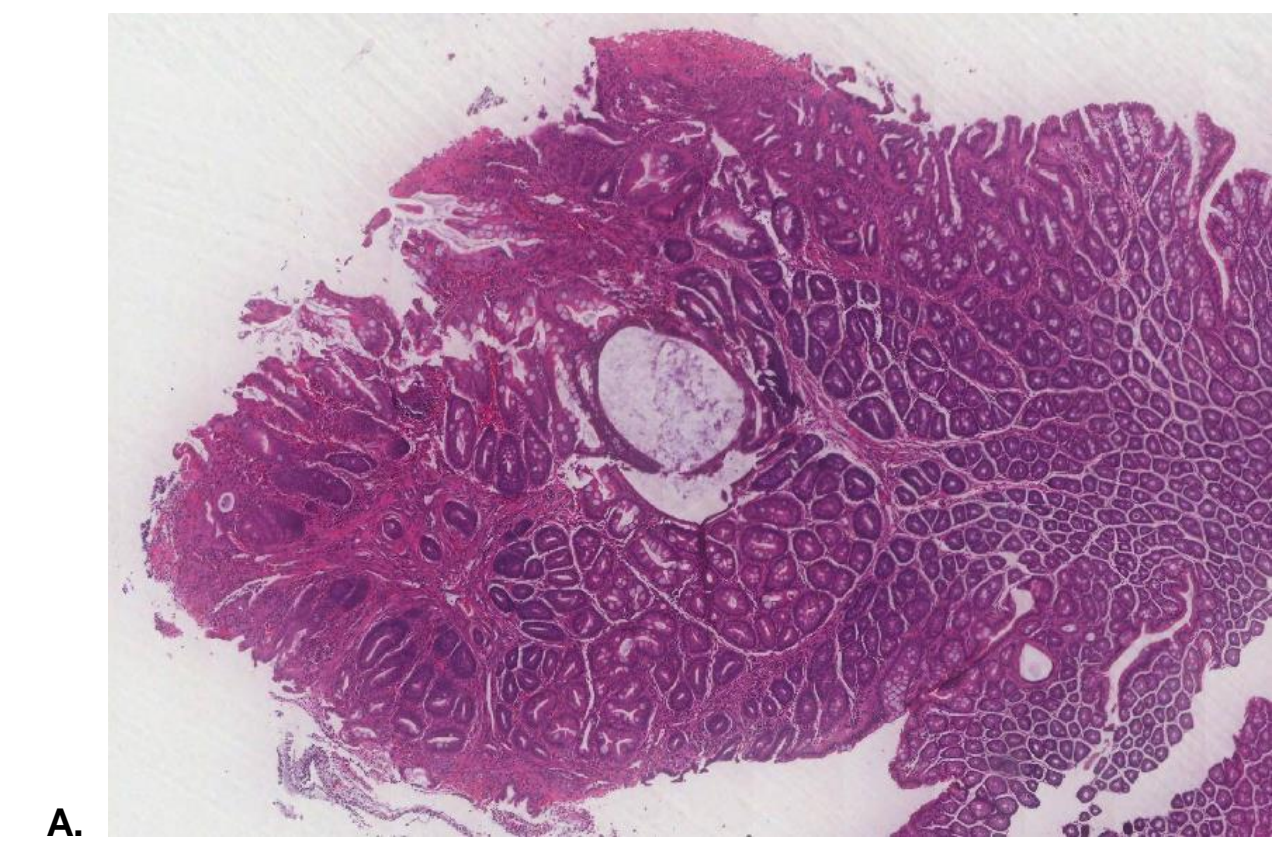

B.

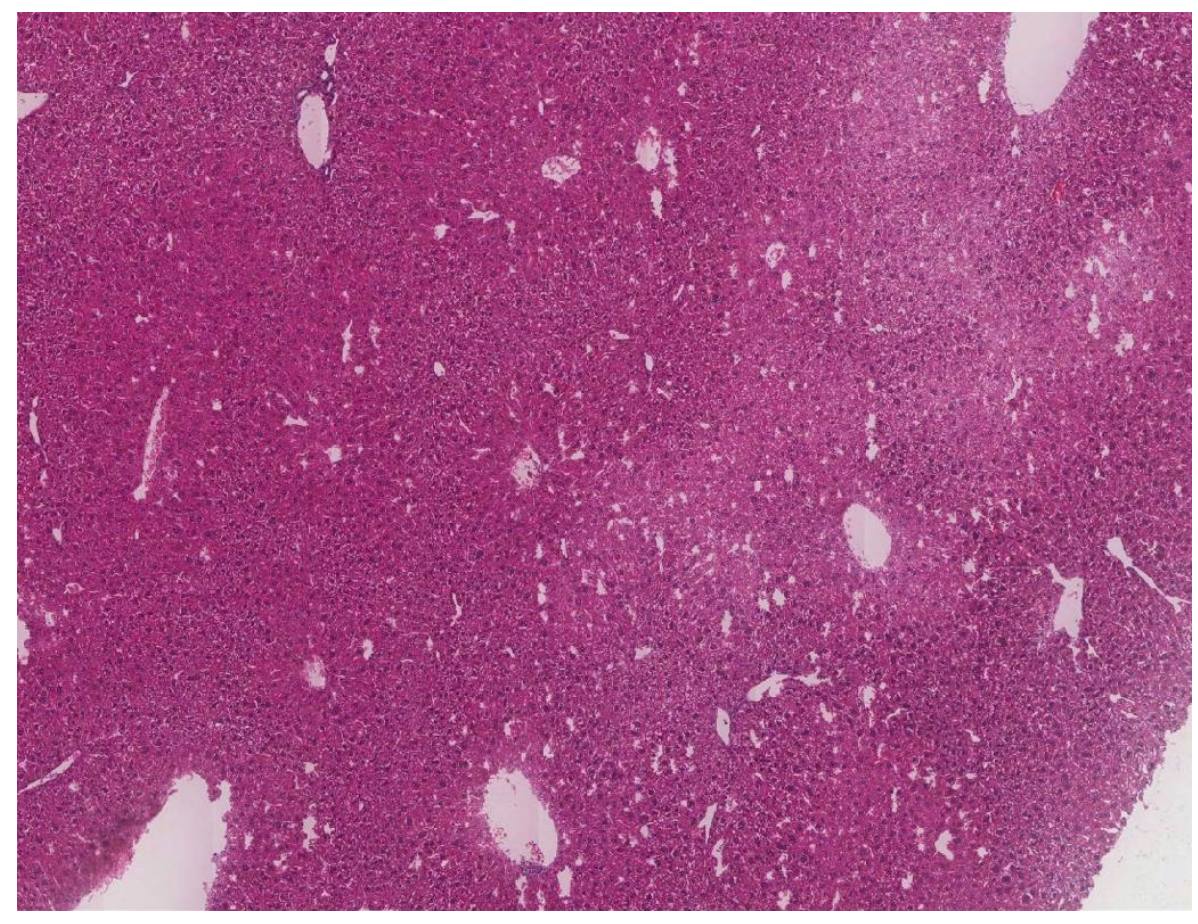

Figure 3: Histologic evaluation of colon tumor, mesenteric lymph nodes, and liver 12 weeks after adenovirus instillation. (A) Histology of colon tumor (4x) demonstrates invasive adenocarcinoma. (B) Representative histology of the liver (4x) showing no evidence of metastases. 


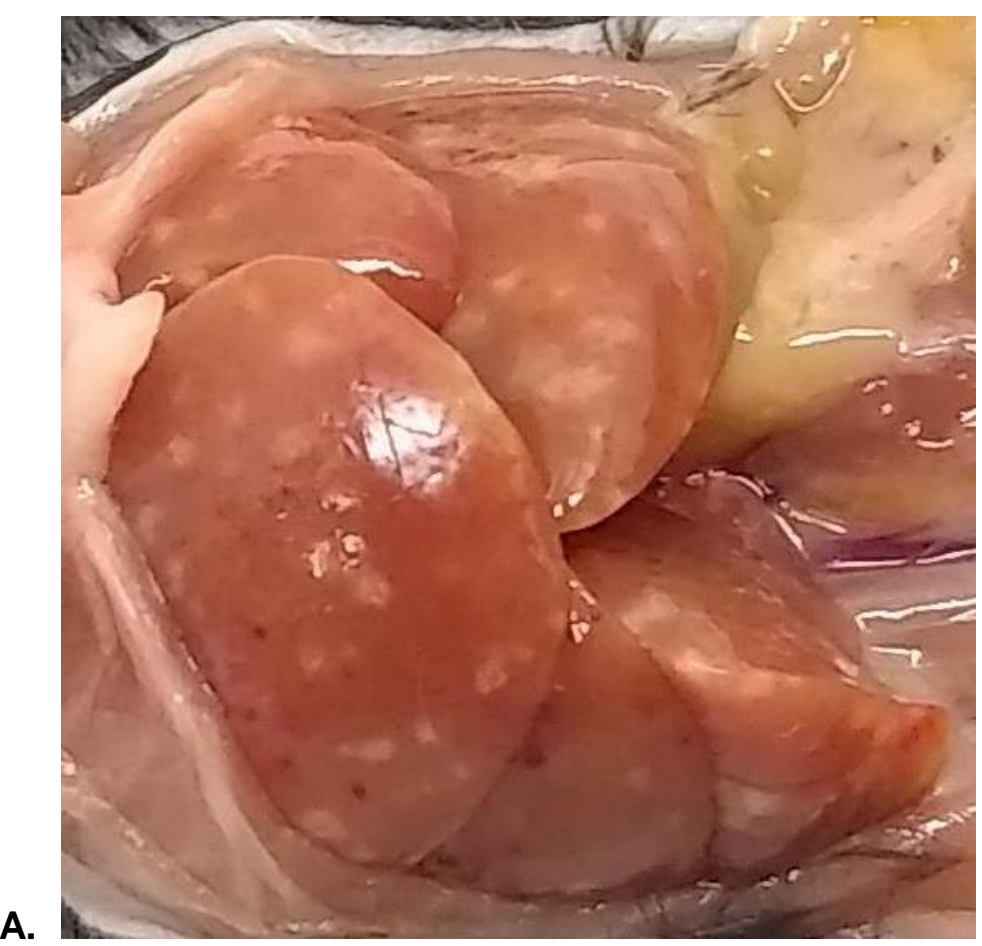

Figure 4: Gross demonstration of colorectal liver metastases 48 weeks after adenovirus instillation. 


\section{OVERALL SUMMARY AND FUTURE DIRECTIONS}

This dissertation began with an investigation into colon inflammation, demonstrating not only its severity in our main model system, but also the ability of a novel, non-invasive imaging modality to identify areas of inflammation in the mouse colon. These findings provided support for application of such technology in evaluation of human subjects at risk for inflammatory bowel disease and colon cancer. The dissertation proceeded to utilize that same imaging technology, together with fluorescence microscopy, to track orally-administered particles. These studies allowed identification of sites of uptake of these particles as well as delineating of the time course of uptake, trafficking, and degradation. These particles were then used as mechanisms of cytokine delivery for oral treatment of colon cancer. Combining oral IL-10 and IL-12 resulted in significant decrease in tumor burden and histologic severity of disease as a result of modulation of CD8+ T cells, RORYT+ IL-17 producing cells, and, most interestingly, colon membrane permeability. Using the same model and drug delivery system, the dissertation proceeded to demonstrate that IL-17 and $ү \delta \mathrm{T}$ cells mediate resistance of colon cancer to anti-PD1 therapy, providing exciting potentials for targeted clinical investigation. The dissertation concluded with description of a feasible preclinical model of spontaneously developing colorectal liver metastases for the evaluation of genetic and immunologic changes associated with development and progression of colon tumors as well as the therapeutic effect of antineoplastic agents in the treatment of metastatic disease. 
Taken together, this body of work incorporates novel technologies, spans a wide range of biologic investigations, offers potential solutions to significant clinical and preclinical problems, and offers exciting opportunities for future lines of investigation. Indeed, as mentioned herein, clinical trials involving MSOT for detection of colon cancer and inflammatory bowel disease, and orally-delivered biodegradable particle-based therapy and combination immunotherapy with anti-IL-17 and anti-PD1/PD-L1 are all imminently realizable in the near future. These, together with opportunities for future studies utilizing the C57BL/6-Apctm1Tyj/J x LSL-K-ras G12D adenovirus-based model, have the potential to significantly impact diagnosis and treatment of colon cancer across all stages of disease, thereby improving quality of life and oncologic outcomes for millions of patients. 


\section{REFERENCES}

\section{Introduction}

1. American Cancer Society. Colorectal Cancer Facts and Figures 2017-2019. Atlanta, GA: American Cancer Society;2017.

2. Surveillance E, and End Results Program. Cancer Stat Facts: Colorectal Cancer. https://seer.cancer.gov/statfacts/html/colorect.html. Accessed 5/9/2018, 2018.

3. Butterworth AS, Higgins JP, Pharoah P. Relative and absolute risk of colorectal cancer for individuals with a family history: a meta-analysis. European journal of cancer (Oxford, England : 1990). 2006;42(2):216-227.

4. Johns LE, Houlston RS. A systematic review and meta-analysis of familial colorectal cancer risk. The American journal of gastroenterology. $2001 ; 96(10): 2992-3003$.

5. Patel SG, Ahnen DJ. Familial colon cancer syndromes: an update of a rapidly evolving field. Current gastroenterology reports. 2012;14(5):428-438.

6. United States National Library of Medicine. Lynch syndrome. Genetics Home Reference https://ghr.nlm.nih.gov/condition/lynch-syndrome\#genes.

7. Bonadona V, Bonaiti B, Olschwang S, et al. Cancer risks associated with germline mutations in MLH1, MSH2, and MSH6 genes in Lynch syndrome. Jama. 2011;305(22):2304-2310.

8. Galiatsatos P, Foulkes WD. Familial adenomatous polyposis. The American journal of gastroenterology. 2006;101(2):385-398. 
9. Lutgens MW, van Oijen MG, van der Heijden GJ, Vleggaar FP, Siersema PD,

Oldenburg B. Declining risk of colorectal cancer in inflammatory bowel disease: an updated meta-analysis of population-based cohort studies. Inflammatory bowel diseases. 2013;19(4):789-799.

10. Beaugerie L, Itzkowitz SH. Cancers Complicating Inflammatory Bowel Disease. New England Journal of Medicine. 2015;372(15):1441-1452.

11. Tsilidis KK, Kasimis JC, Lopez DS, Ntzani EE, loannidis JPA. Type 2 diabetes and cancer: umbrella review of meta-analyses of observational studies. BMJ : British Medical Journal. 2015;350.

12. Kim ER, Chang DK. Colorectal cancer in inflammatory bowel disease: the risk, pathogenesis, prevention and diagnosis. World journal of gastroenterology. 2014;20(29):9872-9881.

13. Fearon ER, Vogelstein B. A genetic model for colorectal tumorigenesis. Cell. 1990;61(5):759-767.

14. Azer SA. Overview of molecular pathways in inflammatory bowel disease associated with colorectal cancer development. European journal of gastroenterology \& hepatology. 2013;25(3):271-281.

15. Triantafillidis JK, Nasioulas G, Kosmidis PA. Colorectal cancer and inflammatory bowel disease: epidemiology, risk factors, mechanisms of carcinogenesis and prevention strategies. Anticancer research. 2009;29(7):2727-2737.

16. Ullman TA, Itzkowitz SH. Intestinal inflammation and cancer. Gastroenterology. 2011;140(6):1807-1816.

17. Jones HA, Clark RJ, Rhodes CG, Schofield JB, Krausz T, Haslett C. In vivo measurement of neutrophil activity in experimental lung inflammation. American journal of respiratory and critical care medicine. 1994;149(6):1635-1639. 
18. Svrcek M, El-Bchiri J, Chalastanis A, et al. Specific clinical and biological features characterize inflammatory bowel disease associated colorectal cancers showing microsatellite instability. Journal of clinical oncology : official journal of the American Society of Clinical Oncology. 2007;25(27):4231-4238.

19. Fleisher AS, Esteller M, Harpaz N, et al. Microsatellite instability in inflammatory bowel disease-associated neoplastic lesions is associated with hypermethylation and diminished expression of the DNA mismatch repair gene, hMLH1. Cancer research. 2000;60(17):4864-4868.

20. Schulmann K, Mori Y, Croog V, et al. Molecular phenotype of inflammatory bowel disease-associated neoplasms with microsatellite instability. Gastroenterology. 2005;129(1):74-85.

21. Jones RM, Devers KJ, Kuzel AJ, Woolf SH. Patient-Reported Barriers to Colorectal Cancer Screening: A Mixed-Methods Analysis. American journal of preventive medicine. 2010;38(5):508-516.

22. Koo JH, Leong RW, Ching J, et al. Knowledge of, attitudes toward, and barriers to participation of colorectal cancer screening tests in the Asia-Pacific region: a multicenter study. Gastrointestinal endoscopy. 2012;76(1):126-135.

23. Pickhardt PJ, Hassan C, Halligan S, Marmo R. Colorectal Cancer: CT Colonography and Colonoscopy for Detection-Systematic Review and MetaAnalysis. Radiology. 2011;259(2):393-405.

24. Alberts SR, Horvath WL, Sternfeld WC, et al. Oxaliplatin, fluorouracil, and leucovorin for patients with unresectable liver-only metastases from colorectal cancer: a North Central Cancer Treatment Group phase II study. Journal of clinical oncology. 2005;23(36):9243-9249.

25. Giacchetti S, Perpoint B, Zidani R, et al. Phase III multicenter randomized trial of oxaliplatin added to chronomodulated fluorouracil-leucovorin as first-line 
treatment of metastatic colorectal cancer. Journal of clinical oncology. $2000 ; 18(1): 136-136$.

26. Goldberg RM, Sargent DJ, Morton RF, et al. A randomized controlled trial of fluorouracil plus leucovorin, irinotecan, and oxaliplatin combinations in patients with previously untreated metastatic colorectal cancer. Journal of Clinical Oncology. 2004;22(1):23-30.

27. Douillard J, Cunningham D, Roth A, et al. Irinotecan combined with fluorouracil compared with fluorouracil alone as first-line treatment for metastatic colorectal cancer: a multicentre randomised trial. The Lancet. 2000;355(9209):1041-1047.

28. Wang CC, Li J. An update on chemotherapy of colorectal liver metastases. World journal of gastroenterology. 2012;18(1):25-33.

29. Bismuth $\mathrm{H}$, Adam R, Lévi $\mathrm{F}$, et al. Resection of nonresectable liver metastases from colorectal cancer after neoadjuvant chemotherapy. Annals of surgery. $1996 ; 224(4): 509$.

30. Andre T, Vernerey D, Mineur L, et al. 3 Versus 6 Months of Oxaliplatin-Based Adjuvant Chemotherapy for Patients With Stage III Colon Cancer: Disease-Free Survival Results From a Randomized, Open-Label, International Duration Evaluation of Adjuvant (IDEA) France, Phase III Trial. Journal of Clinical Oncology. 2018:Jco2017760355.

31. Fong Y, Fortner J, Sun RL, Brennan MF, Blumgart LH. Clinical score for predicting recurrence after hepatic resection for metastatic colorectal cancer: analysis of 1001 consecutive cases. Annals of surgery. 1999;230(3):309.

32. Manfredi S, Lepage C, Hatem C, Coatmeur O, Faivre J, Bouvier AM. Epidemiology and Management of Liver Metastases From Colorectal Cancer. Annals of surgery. 2006;244(2):254-259. 
33. Simmonds P, Primrose J, Colquitt J, Garden O, Poston G, Rees M. Surgical resection of hepatic metastases from colorectal cancer: a systematic review of published studies. British journal of cancer. 2006;94(7):982.

34. Pfannschmidt J, Dienemann $\mathrm{H}$, Hoffmann $\mathrm{H}$. Surgical resection of pulmonary metastases from colorectal cancer: a systematic review of published series. The Annals of thoracic surgery. 2007;84(1):324-338.

35. Golfinopoulos V, Salanti G, Pavlidis N, loannidis JPA. Survival and diseaseprogression benefits with treatment regimens for advanced colorectal cancer: a meta-analysis. The Lancet Oncology. 2007;8(10):898-911.

36. Colucci G, Gebbia V, Paoletti G, et al. Phase III randomized trial of FOLFIRI versus FOLFOX4 in the treatment of advanced colorectal cancer: a multicenter study of the Gruppo Oncologico Dell'Italia Meridionale. Journal of clinical oncology : official journal of the American Society of Clinical Oncology. 2005;23(22):4866-4875.

37. Braun MS, Seymour MT. Balancing the efficacy and toxicity of chemotherapy in colorectal cancer. Therapeutic Advances in Medical Oncology. 2011;3(1):43-52.

38. Hurwitz H, Fehrenbacher L, Novotny W, et al. Bevacizumab plus irinotecan, fluorouracil, and leucovorin for metastatic colorectal cancer. The New England journal of medicine. 2004;350(23):2335-2342.

39. Giantonio BJ, Catalano PJ, Meropol NJ, et al. Bevacizumab in combination with oxaliplatin, fluorouracil, and leucovorin (FOLFOX4) for previously treated metastatic colorectal cancer: results from the Eastern Cooperative Oncology Group Study E3200. Journal of clinical oncology : official journal of the American Society of Clinical Oncology. 2007;25(12):1539-1544.

40. Kabbinavar FF, Hambleton J, Mass RD, Hurwitz HI, Bergsland E, Sarkar S. Combined analysis of efficacy: the addition of bevacizumab to 
fluorouracil/leucovorin improves survival for patients with metastatic colorectal cancer. Journal of clinical oncology : official journal of the American Society of Clinical Oncology. 2005;23(16):3706-3712.

41. Gerber B, von Minckwitz G, Eidtmann H, et al. Surgical outcome after neoadjuvant chemotherapy and bevacizumab: results from the GeparQuinto study (GBG 44). Annals of surgical oncology. 2014;21(8):2517-2524.

42. Hapani S, Sher A, Chu D, Wu S. Increased risk of serious hemorrhage with bevacizumab in cancer patients: a meta-analysis. Oncology. 2010;79(1-2):2738.

43. Ranpura V, Hapani S, Wu S. Treatment-related mortality with bevacizumab in cancer patients: a meta-analysis. Jama. 2011;305(5):487-494.

44. Bokemeyer C, Bondarenko I, Makhson A, et al. Fluorouracil, leucovorin, and oxaliplatin with and without cetuximab in the first-line treatment of metastatic colorectal cancer. Journal of clinical oncology. 2008;27(5):663-671.

45. Van Cutsem E, Köhne C-H, Hitre E, et al. Cetuximab and chemotherapy as initial treatment for metastatic colorectal cancer. New England Journal of Medicine. 2009;360(14):1408-1417.

46. Van Cutsem E. Challenges in the use of epidermal growth factor receptor inhibitors in colorectal cancer. The oncologist. 2006;11(9):1010-1017.

47. Smyth MJ, Hayakawa Y, Takeda K, Yagita H. New aspects of natural-killer-cell surveillance and therapy of cancer. Nature Reviews Cancer. 2002;2:850.

48. Malmberg KJ, Bryceson YT, Carlsten M, et al. NK cell-mediated targeting of human cancer and possibilities for new means of immunotherapy. Cancer immunology, immunotherapy : CII. 2008;57(10):1541-1552. 
49. Coca S, Perez-Piqueras J, Martinez D, et al. The prognostic significance of intratumoral natural killer cells in patients with colorectal carcinoma. Cancer. 1997;79(12):2320-2328.

50. Sandel MH, Speetjens FM, Menon AG, et al. Natural killer cells infiltrating colorectal cancer and MHC class I expression. Molecular immunology. $2005 ; 42(4): 541-546$.

51. Edin S, Wikberg ML, Rutegard J, Oldenborg PA, Palmqvist R. Phenotypic skewing of macrophages in vitro by secreted factors from colorectal cancer cells. PloS one. 2013;8(9):e74982.

52. Keogh E, Fikes J, Southwood S, Celis E, Chesnut R, Sette A. Identification of new epitopes from four different tumor-associated antigens: recognition of naturally processed epitopes correlates with HLA-A*0201-binding affinity. Journal of immunology (Baltimore, Md : 1950). 2001;167(2):787-796.

53. Nagorsen D, Keilholz U, Rivoltini L, et al. Natural T-cell response against MHC class I epitopes of epithelial cell adhesion molecule, her-2/neu, and carcinoembryonic antigen in patients with colorectal cancer. Cancer Res. $2000 ; 60(17): 4850-4854$.

54. Smyrk TC, Watson P, Kaul K, Lynch HT. Tumor-infiltrating lymphocytes are a marker for microsatellite instability in colorectal carcinoma. Cancer. $2001 ; 91(12): 2417-2422$.

55. Michael-Robinson JM, Biemer-Huttmann A, Purdie DM, et al. Tumour infiltrating lymphocytes and apoptosis are independent features in colorectal cancer stratified according to microsatellite instability status. Gut. 2001;48(3):360-366.

56. Pernot S, Terme M, Voron T, et al. Colorectal cancer and immunity: What we know and perspectives. World journal of gastroenterology. 2014;20(14):37383750. 
57. Galon J, Costes A, Sanchez-Cabo F, et al. Type, density, and location of immune cells within human colorectal tumors predict clinical outcome. Science (New York, NY). 2006;313(5795):1960-1964.

58. Mlecnik B, Tosolini M, Kirilovsky A, et al. Histopathologic-based prognostic factors of colorectal cancers are associated with the state of the local immune reaction. Journal of Clinical Oncology. 2011;29(6):610-618.

59. Pages F, Berger A, Camus M, et al. Effector memory $T$ cells, early metastasis, and survival in colorectal cancer. The New England journal of medicine. 2005;353(25):2654-2666.

60. Pages F, Kirilovsky A, Mlecnik B, et al. In situ cytotoxic and memory T cells predict outcome in patients with early-stage colorectal cancer. Journal of Clinical Oncology. 2009;27(35):5944-5951.

61. Tosolini M, Kirilovsky A, Mlecnik B, et al. Clinical impact of different classes of infiltrating T cytotoxic and helper cells (Th1, th2, treg, th17) in patients with colorectal cancer. Cancer Res. 2011;71(4):1263-1271.

62. Allard MA, Bachet JB, Beauchet A, et al. Linear quantification of lymphoid infiltration of the tumor margin: a reproducible method, developed with colorectal cancer tissues, for assessing a highly variable prognostic factor. Diagnostic pathology. 2012;7:156.

63. Tanchot $\mathrm{C}$, Terme $\mathrm{M}$, Pere $\mathrm{H}$, et al. Tumor-infiltrating regulatory $\mathrm{T}$ cells: phenotype, role, mechanism of expansion in situ and clinical significance. Cancer microenvironment : official journal of the International Cancer Microenvironment Society. 2013;6(2):147-157.

64. Facciabene A, Peng X, Hagemann IS, et al. Tumour hypoxia promotes tolerance and angiogenesis via CCL28 and T(reg) cells. Nature. 2011;475(7355):226-230. 
65. Belkaid $\mathrm{Y}$, Oldenhove $\mathrm{G}$. Tuning microenvironments: induction of regulatory $T$ cells by dendritic cells. Immunity. 2008;29(3):362-371 .

66. Ghiringhelli F, Puig PE, Roux S, et al. Tumor cells convert immature myeloid dendritic cells into TGF-beta-secreting cells inducing CD4+CD25+ regulatory $T$ cell proliferation. The Journal of experimental medicine. 2005;202(7):919-929.

67. Terme M, Pernot S, Marcheteau E, et al. VEGFA-VEGFR pathway blockade inhibits tumor-induced regulatory T-cell proliferation in colorectal cancer. Cancer Res. 2013;73(2):539-549.

68. Casares N, Arribillaga L, Sarobe $\mathrm{P}$, et al. $\mathrm{CD} 4+/ \mathrm{CD} 25+$ regulatory cells inhibit activation of tumor-primed CD4+ T cells with IFN-gamma-dependent antiangiogenic activity, as well as long-lasting tumor immunity elicited by peptide vaccination. Journal of immunology (Baltimore, Md : 1950). 2003;171(11):59315939.

69. Yaqub S, Henjum K, Mahic M, et al. Regulatory T cells in colorectal cancer patients suppress anti-tumor immune activity in a COX-2 dependent manner. Cancer immunology, immunotherapy : CII. 2008;57(6):813-821.

70. Bonertz A, Weitz J, Pietsch DH, et al. Antigen-specific Tregs control T cell responses against a limited repertoire of tumor antigens in patients with colorectal carcinoma. The Journal of clinical investigation. 2009;119(11):33113321.

71. Vincent J, Mignot G, Chalmin F, et al. 5-Fluorouracil selectively kills tumorassociated myeloid-derived suppressor cells resulting in enhanced T celldependent antitumor immunity. Cancer Res. 2010;70(8):3052-3061.

72. Shi SJ, Wang LJ, Wang GD, et al. B7-H1 expression is associated with poor prognosis in colorectal carcinoma and regulates the proliferation and invasion of HCT116 colorectal cancer cells. PloS one. 2013;8(10):e76012. 
73. Hua D, Sun J, Mao Y, Chen LJ, Wu YY, Zhang XG. B7-H1 expression is associated with expansion of regulatory T cells in colorectal carcinoma. World journal of gastroenterology. 2012;18(9):971-978.

74. Wu S, Rhee KJ, Albesiano E, et al. A human colonic commensal promotes colon tumorigenesis via activation of $\mathrm{T}$ helper type $17 \mathrm{~T}$ cell responses. Nature medicine. 2009;15(9):1016-1022.

75. Housseau F, Wu S, Wick EC, et al. Redundant innate and adaptive sources of IL-17 production drive colon tumorigenesis. Cancer Res. 2016;76(8):2115-2124.

76. Wu P, Wu D, Ni C, et al. gammadeltaT17 cells promote the accumulation and expansion of myeloid-derived suppressor cells in human colorectal cancer. Immunity. 2014;40(5):785-800.

77. Grivennikov SI, Wang K, Mucida D, et al. Adenoma-linked barrier defects and microbial products drive IL-23/IL-17-mediated tumour growth. Nature. 2012;491(7423):254-258.

78. Wang K, Kim MK, Di Caro G, et al. Interleukin-17 receptor a signaling in transformed enterocytes promotes early colorectal tumorigenesis. Immunity. 2014;41(6):1052-1063.

79. Luissint AC, Parkos CA, Nusrat A. Inflammation and the Intestinal Barrier: Leukocyte-Epithelial Cell Interactions, Cell Junction Remodeling, and Mucosal Repair. Gastroenterology. 2016;151(4):616-632.

80. Correale P, Cusi MG, Tsang KY, et al. Chemo-immunotherapy of metastatic colorectal carcinoma with gemcitabine plus FOLFOX 4 followed by subcutaneous granulocyte macrophage colony-stimulating factor and interleukin2 induces strong immunologic and antitumor activity in metastatic colon cancer patients. Journal of Clinical Oncology. 2005;23(35):8950-8958. 
81. Correale P, Tagliaferri P, Fioravanti A, et al. Immunity feedback and clinical outcome in colon cancer patients undergoing chemoimmunotherapy with gemcitabine + FOLFOX followed by subcutaneous granulocyte macrophage colony-stimulating factor and aldesleukin (GOLFIG-1 Trial). Clinical cancer research : an official journal of the American Association for Cancer Research. 2008;14(13):4192-4199.

82. Correale P, Botta C, Rotundo MS, et al. Gemcitabine, oxaliplatin, levofolinate, 5fluorouracil, granulocyte-macrophage colony-stimulating factor, and interleukin-2 (GOLFIG) versus FOLFOX chemotherapy in metastatic colorectal cancer patients: the GOLFIG-2 multicentric open-label randomized phase III trial. Journal of immunotherapy (Hagerstown, Md : 1997). 2014;37(1):26-35.

83. Vermorken JB, Claessen AM, van Tinteren $\mathrm{H}$, et al. Active specific immunotherapy for stage II and stage III human colon cancer: a randomised trial. Lancet (London, England). 1999;353(9150):345-350.

84. Harris JE, Ryan L, Hoover HC, Jr., et al. Adjuvant active specific immunotherapy for stage II and III colon cancer with an autologous tumor cell vaccine: Eastern Cooperative Oncology Group Study E5283. Journal of Clinical Oncology. $2000 ; 18(1): 148-157$.

85. Overman MJ, Lonardi S, Wong KYM, et al. Durable Clinical Benefit With Nivolumab Plus Ipilimumab in DNA Mismatch Repair-Deficient/Microsatellite Instability-High Metastatic Colorectal Cancer. Journal of Clinical Oncology. 2018;36(8):773-779.

86. Overman MJ, McDermott R, Leach JL, et al. Nivolumab in patients with metastatic DNA mismatch repair-deficient or microsatellite instability-high colorectal cancer (CheckMate 142): an open-label, multicentre, phase 2 study. The Lancet Oncology. 2017;18(9):1182-1191. 
87. Le DT, Uram JN, Wang H, et al. PD-1 Blockade in Tumors with Mismatch-Repair Deficiency. The New England journal of medicine. 2015;372(26):2509-2520.

88. Topalian SL, Hodi FS, Brahmer JR, et al. Safety, Activity, and Immune Correlates of Anti-PD-1 Antibody in Cancer. The New England journal of medicine. 2012;366(26):2443-2454.

89. Tauriello DVF, Palomo-Ponce S, Stork D, et al. TGF $\beta$ drives immune evasion in genetically reconstituted colon cancer metastasis. Nature. 2018;554:538.

90. Shin DS, Zaretsky JM, Escuin-Ordinas H, et al. Primary Resistance to PD-1 Blockade Mediated by <em>JAK1/2</em> Mutations. Cancer Discovery. 2017;7(2):188-201.

\section{Chapter One}

1. Molodecky NA, Soon IS, Rabi DM, et al. Increasing incidence and prevalence of the inflammatory bowel diseases with time, based on systematic review. Gastroenterology. 2012;142(1):46-54.e42; quiz e30.

2. Tontini GE, Vecchi M, Pastorelli L, Neurath MF, Neumann H. Differential diagnosis in inflammatory bowel disease colitis: state of the art and future perspectives. World journal of gastroenterology. 2015;21(1):21-46.

3. Mattar MC, Lough D, Pishvaian MJ, Charabaty A. Current management of inflammatory bowel disease and colorectal cancer. Gastrointestinal cancer research : GCR. 2011;4(2):53-61.

4. Ntziachristos V, Razansky D. Optical and opto-acoustic imaging. Recent results in cancer research Fortschritte der Krebsforschung Progres dans les recherches sur le cancer. 2013;187:133-150. 
5. Bhutiani N, Kimbrough CW, Burton NC, et al. Detection of microspheres in vivo using multispectral optoacoustic tomography. Biotechnic \& histochemistry : official publication of the Biological Stain Commission. 2017;92(1):1-6.

6. Kimbrough CW, Khanal A, Zeiderman M, et al. Targeting acidity in pancreatic adenocarcinoma: multispectral optoacoustic tomography detects ph-low insertion peptide probes in vivo. Clinical Cancer Research. 2015;21(20):45764585.

7. McNally LR, Mezera M, Morgan DE, et al. Current and Emerging Clinical Applications of Multispectral Optoacoustic Tomography (MSOT) in Oncology. Clinical Cancer Research. 2016.

8. Burton NC, Patel M, Morscher S, et al. Multispectral opto-acoustic tomography (MSOT) of the brain and glioblastoma characterization. Neurolmage. $2013 ; 65: 522-528$.

9. Cao J, Campbell J, Liu L, Mason RP, Lippert AR. In Vivo Chemiluminescent Imaging Agents for Nitroreductase and Tissue Oxygenation. Analytical Chemistry. 2016;88(9):4995-5002.

10. Waldner MJ, Knieling F, Egger C, et al. Multispectral Optoacoustic Tomography in Crohn's Disease: Noninvasive Imaging of Disease Activity. Gastroenterology. 2016;151(2):238-240.

11. Razansky D, Buehler A, Ntziachristos V. Volumetric real-time multispectral optoacoustic tomography of biomarkers. Nature Protocols. 2011;6:1121.

12. Ma R, Taruttis A, Ntziachristos V, Razansky D. Multispectral optoacoustic tomography (MSOT) scanner for whole-body small animal imaging. Optics express. 2009;17(24):21414-21426.

13. Dietrich O, Raya JG, Reeder SB, Reiser MF, Schoenberg SO. Measurement of signal-to-noise ratios in MR images: influence of multichannel coils, parallel 
imaging, and reconstruction filters. Journal of magnetic resonance imaging : JMRI. 2007;26(2):375-385.

14. Rabizadeh S, Rhee KJ, Wu S, et al. Enterotoxigenic bacteroides fragilis: a potential instigator of colitis. Inflammatory bowel diseases. 2007;13(12):14751483.

15. Rhee KJ, Wu S, Wu X, et al. Induction of persistent colitis by a human commensal, enterotoxigenic Bacteroides fragilis, in wild-type C57BL/6 mice. Infection and immunity. 2009;77(4):1708-1718.

16. Becker C, Fantini MC, Neurath MF. High resolution colonoscopy in live mice. Nature protocols. 2006;1(6):2900-2904.

17. Buehler A, Kacprowicz M, Taruttis A, Ntziachristos V. Real-time handheld multispectral optoacoustic imaging. Opt Lett. 2013;38(9):1404-1406.

18. Stoffels I, Morscher S, Helfrich I, et al. Metastatic status of sentinel lymph nodes in melanoma determined noninvasively with multispectral optoacoustic imaging. Science translational medicine. 2015;7(317):317ra199.

\section{Chapter Two}

1. Chung AY, Li Q, Blair SJ, et al. Oral interleukin-10 alleviates polyposis via neutralization of pathogenic T-regulatory cells. Cancer research. 2014;74(19):5377-5385.

2. Egilmez NK, Jong YS, Iwanuma Y, et al. Cytokine immunotherapy of cancer with controlled release biodegradable microspheres in a human tumor xenograft/SCID mouse model. Cancer immunology, immunotherapy : CII. 1998;46(1):21-24. 
3. Egilmez NK, Kilinc MO, Gu T, Conway TF. Controlled-release particulate cytokine adjuvants for cancer therapy. Endocrine, metabolic \& immune disorders drug targets. 2007;7(4):266-270.

4. Sabel MS, Skitzki J, Stoolman L, et al. Intratumoral IL-12 and TNF-alpha-loaded microspheres lead to regression of breast cancer and systemic antitumor immunity. Annals of surgical oncology. 2004;11(2):147-156.

5. Sharma A, Harper CM, Hammer L, Nair RE, Mathiowitz E, Egilmez NK. Characterization of cytokine-encapsulated controlled-release microsphere adjuvants. Cancer biotherapy \& radiopharmaceuticals. 2004;19(6):764-769.

6. Beziere N, Lozano N, Nunes A, et al. Dynamic imaging of PEGylated indocyanine green (ICG) liposomes within the tumor microenvironment using multi-spectral optoacoustic tomography (MSOT). Biomaterials. 2015;37:415-424.

7. Buehler A, Herzog E, Ale A, Smith BD, Ntziachristos V, Razansky D. High resolution tumor targeting in living mice by means of multispectral optoacoustic tomography. EJNMMI research. 2012;2:14.

8. Burton NC, Patel M, Morscher S, et al. Multispectral opto-acoustic tomography (MSOT) of the brain and glioblastoma characterization. Neurolmage. 2013;65:522-528.

9. Herzog E, Taruttis A, Beziere N, Lutich AA, Razansky D, Ntziachristos V. Optical imaging of cancer heterogeneity with multispectral optoacoustic tomography. Radiology. 2012;263(2):461-468.

10. Kimbrough CW, Hudson S, Khanal A, Egger ME, McNally LR. Orthotopic pancreatic tumors detected by optoacoustic tomography using Syndecan-1. The Journal of surgical research. 2015;193(1):246-254.

11. Neuschmelting V, Lockau H, Ntziachristos V, Grimm J, Kircher MF. Lymph Node Micrometastases and In-Transit Metastases from Melanoma: In Vivo Detection 
with Multispectral Optoacoustic Imaging in a Mouse Model. Radiology. 2016;280(1):137-150.

12. Mason RP. Commentary on Photoacoustic Tomography. Journal of Nuclear Medicine. 2015;56(12):1815-1816.

13. Conway TF, Hammer L, Furtado S, et al. Oral Delivery of Particulate Transforming Growth Factor Beta 1 and All-Trans Retinoic Acid Reduces Gut Inflammation in Murine Models of Inflammatory Bowel Disease. Journal of Crohn's \& colitis. 2015;9(8):647-658.

14. Egilmez NK, Jong YS, Mathiowitz E, Bankert RB. Tumor vaccination with cytokine-encapsulated microspheres. Lung Cancer: Springer; 2003:687-696.

15. Gu T, De Jesus M, Gallagher HC, Burris TP, Egilmez NK. Oral IL-10 suppresses colon carcinogenesis via elimination of pathogenicCD4(+) T-cells and induction of antitumor CD8(+) T-cell activity. Oncoimmunology. 2017;6(6):e1319027.

16. Bhutiani N, Grizzle WE, Galandiuk S, et al. Noninvasive Imaging of Colitis Using Multispectral Optoacoustic Tomography. Journal of nuclear medicine : official publication, Society of Nuclear Medicine. 2017;58(6):1009-1012.

17. Kimbrough CW, Khanal A, Zeiderman M, et al. Targeting acidity in pancreatic adenocarcinoma: multispectral optoacoustic tomography detects ph-low insertion peptide probes in vivo. Clinical Cancer Research. 2015;21(20):45764585.

18. Hudson SV, Huang JS, Yin W, et al. Targeted noninvasive imaging of EGFRexpressing orthotopic pancreatic cancer using multispectral optoacoustic tomography. Cancer research. 2014;74(21):6271-6279.

19. Tzoumas S, Deliolanis N, Morscher S, Ntziachristos V. Un-mixing Molecular Agents from Absorbing Tissue in Multispectral Optoacoustic Tomography. IEEE transactions on medical imaging. 2013. 
20. McNally LR, Mezera M, Morgan DE, et al. Current and Emerging Clinical Applications of Multispectral Optoacoustic Tomography (MSOT) in Oncology. Clinical Cancer Research. 2016.

21. Morscher S, Driessen WH, Claussen J, Burton NC. Semi-quantitative Multispectral Optoacoustic Tomography (MSOT) for volumetric PK imaging of gastric emptying. Photoacoustics. Vol 2. Germany2014:103-110.

22. Bhutiani N, Kimbrough CW, Burton NC, et al. Detection of microspheres in vivo using multispectral optoacoustic tomography. Biotechnic \& histochemistry. 2017;92(1):1-6.

23. Gurka MK, Pender D, Chuong $\mathrm{P}$, et al. Identification of pancreatic tumors in vivo with ligand-targeted, $\mathrm{pH}$ responsive mesoporous silica nanoparticles by multispectral optoacoustic tomography. Journal of controlled release : official journal of the Controlled Release Society. 2016;231:60-67.

24. Fraser JR, Kimpton WG, Laurent TC, Cahill RN, Vakakis N. Uptake and degradation of hyaluronan in lymphatic tissue. The Biochemical journal. 1988;256(1):153-158.

25. Waldner MJ, Knieling F, Egger C, et al. Multispectral Optoacoustic Tomography in Crohn's Disease: Noninvasive Imaging of Disease Activity. Gastroenterology. 2016;151(2):238-240.

\section{Chapter Three}

1. Ribas, A., \& Wolchok, J. D. (2018). Cancer immunotherapy using checkpoint blockade. Science, 359(6382), pp. 1350-1355. doi:10.1126/science.aar4060

2. Queirolo, P., \& Spagnolo, F. (2017). Atypical responses in patients with advanced melanoma, lung cancer, renal-cell carcinoma and other solid tumors 
treated with anti-PD-1 drugs: A systematic review. Cancer Treat Rev, 59, pp. 7178. doi:10.1016/j.ctrv.2017.07.002

3. Boland, P. M., \& Ma, W. W. (2017). Immunotherapy for Colorectal Cancer. Cancers (Basel), 9(5)doi:10.3390/cancers9050050

4. Zhang, K., Hornef, M. W., \& Dupont, A. (2015). The intestinal epithelium as guardian of gut barrier integrity. Cell Microbiol, 17(11), pp. 1561-1569. doi:10.1111/cmi.12501

5. de Souza, H. S., \& Fiocchi, C. (2016). Immunopathogenesis of IBD: current state of the art. Nat Rev Gastroenterol Hepatol, 13(1), pp. 13-27. doi:10.1038/nrgastro.2015.186

6. Kang, M., \& Martin, A. (2017). Microbiome and colorectal cancer: Unraveling host-microbiota interactions in colitis-associated colorectal cancer development. Semin Immunol, 32, pp. 3-13. doi:10.1016/j.smim.2017.04.003

7. Grivennikov, S. I., Wang, K., Mucida, D., Stewart, C. A., Schnabl, B., Jauch, D., Taniguchi, K., Yu, G. Y., Osterreicher, C. H., Hung, K. E., et al. (2012). Adenoma-linked barrier defects and microbial products drive IL-23/IL-17mediated tumour growth. Nature, 491(7423), pp. 254-258.

doi:10.1038/nature11465

8. Wu, S., Rhee, K. J., Albesiano, E., Rabizadeh, S., Wu, X., Yen, H. R., Huso, D. L., Brancati, F. L., Wick, E., McAllister, F., et al. (2009). A human colonic commensal promotes colon tumorigenesis via activation of $\mathrm{T}$ helper type $17 \mathrm{~T}$ cell responses. Nat Med, 15(9), pp. 1016-1022. doi:10.1038/nm.2015

9. Blatner, N. R., Mulcahy, M. F., Dennis, K. L., Scholtens, D., Bentrem, D. J., Phillips, J. D., Ham, S., Sandall, B. P., Khan, M. W., Mahvi, D. M., et al. (2012). Expression of RORgammat marks a pathogenic regulatory $\mathrm{T}$ cell subset in 
human colon cancer. Sci Trans/ Med, 4(164), p 164ra159.

doi:10.1126/scitransImed.3004566

10. Wang, K., Kim, M. K., Di Caro, G., Wong, J., Shalapour, S., Wan, J., Zhang, W., Zhong, Z., Sanchez-Lopez, E., Wu, L. W., et al. (2014). Interleukin-17 receptor a signaling in transformed enterocytes promotes early colorectal tumorigenesis. Immunity, 41(6), pp. 1052-1063. doi:10.1016/j.immuni.2014.11.009

11. Le Gouvello, S., Bastuji-Garin, S., Aloulou, N., Mansour, H., Chaumette, M. T., Berrehar, F., Seikour, A., Charachon, A., Karoui, M., Leroy, K., et al. (2008). High prevalence of Foxp3 and IL17 in MMR-proficient colorectal carcinomas. Gut, 57(6), pp. 772-779. doi:10.1136/gut.2007.123794

12. Sabat, R., Grutz, G., Warszawska, K., Kirsch, S., Witte, E., Wolk, K., \& Geginat, J. (2010). Biology of interleukin-10. Cytokine Growth Factor Rev, 21(5), pp. 331 344. doi:10.1016/j.cytogfr.2010.09.002

13. Shouval, D. S., Biswas, A., Goettel, J. A., McCann, K., Conaway, E., Redhu, N. S., Mascanfroni, I. D., Al Adham, Z., Lavoie, S., Ibourk, M., et al. (2014). Interleukin-10 receptor signaling in innate immune cells regulates mucosal immune tolerance and anti-inflammatory macrophage function. Immunity, 4O(5), pp. 706-719. doi:10.1016/j.immuni.2014.03.011

14. Zigmond, E., Bernshtein, B., Friedlander, G., Walker, C. R., Yona, S., Kim, K. W., Brenner, O., Krauthgamer, R., Varol, C., Muller, W., et al. (2014). Macrophagerestricted interleukin-10 receptor deficiency, but not IL-10 deficiency, causes severe spontaneous colitis. Immunity, 40(5), pp. 720-733. doi:10.1016/j.immuni.2014.03.012

15. Ip, W. K. E., Hoshi, N., Shouval, D. S., Snapper, S., \& Medzhitov, R. (2017). Antiinflammatory effect of IL-10 mediated by metabolic reprogramming of macrophages. Science, 356(6337), pp. 513-519. doi:10.1126/science.aal3535 
16. Huber, S., Gagliani, N., Esplugues, E., O'Connor, W., Jr., Huber, F. J., Chaudhry, A., Kamanaka, M., Kobayashi, Y., Booth, C. J., Rudensky, A. Y., et al. (2011). Th17 cells express interleukin-10 receptor and are controlled by Foxp3(-) and Foxp3+ regulatory CD4+ T cells in an interleukin-10-dependent manner. Immunity, 34(4), pp. 554-565. doi:10.1016/j.immuni.2011.01.020

17. Chaudhry, A., Samstein, R. M., Treuting, P., Liang, Y., Pils, M. C., Heinrich, J. M., Jack, R. S., Wunderlich, F. T., Bruning, J. C., Muller, W., et al. (2011). Interleukin-10 signaling in regulatory T cells is required for suppression of Th17 cell-mediated inflammation. Immunity, 34(4), pp. 566-578. doi:10.1016/j.immuni.2011.03.018

18. Mumm, J. B., Emmerich, J., Zhang, X., Chan, I., Wu, L., Mauze, S., Blaisdell, S., Basham, B., Dai, J., Grein, J., et al. (2011). IL-10 elicits IFNgamma-dependent tumor immune surveillance. Cancer Cell, 20(6), pp. 781-796. doi:10.1016/j.ccr.2011.11.003

19. Emmerich, J., Mumm, J. B., Chan, I. H., LaFace, D., Truong, H., McClanahan, T., Gorman, D. M., \& Oft, M. (2012). IL-10 directly activates and expands tumorresident CD8(+) T cells without de novo infiltration from secondary lymphoid organs. Cancer Res, 72(14), pp. 3570-3581. doi:10.1158/0008-5472.can-120721

20. Chan, I. H., Wu, V., Bilardello, M., Mar, E., Oft, M., Van Vlasselaer, P., \& Mumm, J. B. (2015). The Potentiation of IFN-gamma and Induction of Cytotoxic Proteins by Pegylated IL-10 in Human CD8 T Cells. J Interferon Cytokine Res, 35(12), pp. 948-955. doi:10.1089/jir.2014.0221

21. Chung, A. Y., Li, Q., Blair, S. J., De Jesus, M., Dennis, K. L., LeVea, C., Yao, J., Sun, Y., Conway, T. F., Virtuoso, L. P., et al. (2014). Oral interleukin-10 alleviates 
polyposis via neutralization of pathogenic T-regulatory cells. Cancer Res, 74(19), pp. 5377-5385. doi:10.1158/0008-5472.can-14-0918

22. Gu, T., De Jesus, M., Gallagher, H. C., Burris, T. P., \& Egilmez, N. K. (2017). Oral IL-10 suppresses colon carcinogenesis via elimination of pathogenicCD4(+) T-cells and induction of antitumor CD8(+) T-cell activity. Oncoimmunology, 6(6), p e1319027. doi:10.1080/2162402x.2017.1319027

23. Trinchieri, G. (2003). Interleukin-12 and the regulation of innate resistance and adaptive immunity. Nat Rev Immunol, 3(2), pp. 133-146. doi:10.1038/nri1001

24. Capaldo, C. T., \& Nusrat, A. (2009). Cytokine regulation of tight junctions. Biochim Biophys Acta, 1788(4), pp. 864-871. doi:10.1016/j.bbamem.2008.08.027

25. Brandl, K., Rutschmann, S., Li, X., Du, X., Xiao, N., Schnabl, B., Brenner, D. A., \& Beutler, B. (2009). Enhanced sensitivity to DSS colitis caused by a hypomorphic Mbtps1 mutation disrupting the ATF6-driven unfolded protein response. Proc Natl Acad Sci U S A, 106(9), pp. 3300-3305.

doi:10.1073/pnas.0813036106

26. Tanaka, T., Kohno, H., Suzuki, R., Hata, K., Sugie, S., Niho, N., Sakano, K., Takahashi, M., \& Wakabayashi, K. (2006). Dextran sodium sulfate strongly promotes colorectal carcinogenesis in $\mathrm{Apc}(\mathrm{Min} /+)$ mice: inflammatory stimuli by dextran sodium sulfate results in development of multiple colonic neoplasms. Int J Cancer, 118(1), pp. 25-34. doi:10.1002/ijc.21282

27. Oshima, T., Laroux, F. S., Coe, L. L., Morise, Z., Kawachi, S., Bauer, P., Grisham, M. B., Specian, R. D., Carter, P., Jennings, S., et al. (2001). Interferongamma and interleukin-10 reciprocally regulate endothelial junction integrity and barrier function. Microvasc Res, 61(1), pp. 130-143. doi:10.1006/mvre.2000.2288

28. Madsen, K. L., Lewis, S. A., Tavernini, M. M., Hibbard, J., \& Fedorak, R. N. (1997). Interleukin 10 prevents cytokine-induced disruption of T84 monolayer 
barrier integrity and limits chloride secretion. Gastroenterology, 113(1), pp. 151159.

29. Sun, X., Yang, H., Nose, K., Nose, S., Haxhija, E. Q., Koga, H., Feng, Y., \& Teitelbaum, D. H. (2008). Decline in intestinal mucosal IL-10 expression and decreased intestinal barrier function in a mouse model of total parenteral nutrition. Am J Physiol Gastrointest Liver Physiol, 294(1), pp. G139-147. doi:10.1152/ajpgi.00386.2007

30. Kominsky, D. J., Campbell, E. L., Ehrentraut, S. F., Wilson, K. E., Kelly, C. J., Glover, L. E., Collins, C. B., Bayless, A. J., Saeedi, B., Dobrinskikh, E., et al. (2014). IFN-y-mediated induction of an apical IL-10 receptor on polarized intestinal epithelia. J Immunol, 192(3), pp. 1267-1276. doi:10.4049/jimmunol.1301757

31. Schaefer, A., Unterberger, C., Frankenberger, M., Lohrum, M., Staples, K. J., Werner, T., Stunnenberg, H., \& Ziegler-Heitbrock, L. (2009). Mechanism of interferon-gamma mediated down-regulation of interleukin-10 gene expression. Mol Immunol, 46(7), pp. 1351-1359. doi:10.1016/j.molimm.2008.11.015

32. Luissint, A. C., Parkos, C. A., \& Nusrat, A. (2016). Inflammation and the Intestinal Barrier: Leukocyte-Epithelial Cell Interactions, Cell Junction Remodeling, and Mucosal Repair. Gastroenterology, 151(4), pp. 616-632. doi:10.1053/j.gastro.2016.07.008

33. Lechuga, S., \& Ivanov, A. I. (2017). Disruption of the epithelial barrier during intestinal inflammation: Quest for new molecules and mechanisms. Biochim Biophys Acta, 1864(7), pp. 1183-1194. doi:10.1016/j.bbamcr.2017.03.007

34. Thiele Orberg, E., Fan, H., Tam, A. J., Dejea, C. M., Destefano Shields, C. E., Wu, S., Chung, L., Finard, B. B., Wu, X., Fathi, P., et al. (2017). The myeloid 
immune signature of enterotoxigenic Bacteroides fragilis-induced murine colon tumorigenesis. Mucosal Immunol, 10(2), pp. 421-433. doi:10.1038/mi.2016.53

35. Ito, S., Ansari, P., Sakatsume, M., Dickensheets, H., Vazquez, N., Donnelly, R. P., Larner, A. C., \& Finbloom, D. S. (1999). Interleukin-10 inhibits expression of both interferon alpha- and interferon gamma- induced genes by suppressing tyrosine phosphorylation of STAT1. Blood, 93(5), pp. 1456-1463.

36. Conway, T. F., Hammer, L., Furtado, S., Mathiowitz, E., Nicoletti, F., Mangano, K., Egilmez, N. K., \& Auci, D. L. (2015). Oral Delivery of Particulate Transforming Growth Factor Beta 1 and All-Trans Retinoic Acid Reduces Gut Inflammation in Murine Models of Inflammatory Bowel Disease. J Crohns Colitis, 9(8), pp. 647658. doi:10.1093/ecco-jcc/jjv089

37. Egilmez, N. K., Jong, Y. S., Mathiowitz, E., \& Bankert, R. B. (2003). Tumor vaccination with cytokine-encapsulated microspheres Lung Cancer (pp. 687696): Springer.

38. De Jesus, M., Ahlawat, S., \& Mantis, N. J. (2013). Isolating and immunostaining lymphocytes and dendritic cells from murine Peyer's patches. Journal of visualized experiments: JoVE(73)

39. Schindelin, J., Arganda-Carreras, I., Frise, E., Kaynig, V., Longair, M., Pietzsch, T., Preibisch, S., Rueden, C., Saalfeld, S., \& Schmid, B. (2012). Fiji: an opensource platform for biological-image analysis. Nature methods, 9(7), p 676.

40. Gupta, J., \& Nebreda, A. R. (2014). Analysis of intestinal permeability in mice. Bio-protocol, 4(22)

\section{Chapter Four}


1. Postow MA, Callahan MK, Wolchok JD. Immune Checkpoint Blockade in Cancer Therapy. Journal of Clinical Oncology. 2015;33(17):1974-1982.

2. Ribas A, Wolchok JD. Cancer immunotherapy using checkpoint blockade. Science (New York, NY). 2018;359(6382):1350-1355.

3. Sharma P, Allison James P. Immune Checkpoint Targeting in Cancer Therapy: Toward Combination Strategies with Curative Potential. Cell. 2015;161(2):205214.

4. Topalian Suzanne L, Drake Charles G, Pardoll Drew M. Immune Checkpoint Blockade: A Common Denominator Approach to Cancer Therapy. Cancer Cell. 2015;27(4):450-461.

5. Pardoll DM. The blockade of immune checkpoints in cancer immunotherapy. Nature Reviews Cancer. 2012;12(4):252.

6. Rizvi NA, Mazières J, Planchard D, et al. Activity and safety of nivolumab, an anti-PD-1 immune checkpoint inhibitor, for patients with advanced, refractory squamous non-small-cell lung cancer (CheckMate 063): a phase 2, single-arm trial. The Lancet Oncology. 2015;16(3):257-265.

7. Massard C, Gordon MS, Sharma S, et al. Safety and Efficacy of Durvalumab (MEDI4736), an Anti-Programmed Cell Death Ligand-1 Immune Checkpoint Inhibitor, in Patients With Advanced Urothelial Bladder Cancer. Journal of Clinical Oncology. 2016;34(26):3119-3125.

8. Weber JS, D'Angelo SP, Minor D, et al. Nivolumab versus chemotherapy in patients with advanced melanoma who progressed after anti-CTLA-4 treatment (CheckMate 037): a randomised, controlled, open-label, phase 3 trial. The Lancet Oncology. 2015;16(4):375-384.

9. Wolchok JD, Kluger H, Callahan MK, et al. Nivolumab plus ipilimumab in advanced melanoma. New England Journal of Medicine. 2013;369(2):122-133. 
10. Hodi FS, Chesney J, Pavlick AC, et al. Combined nivolumab and ipilimumab versus ipilimumab alone in patients with advanced melanoma: 2-year overall survival outcomes in a multicentre, randomised, controlled, phase 2 trial. The Lancet Oncology. 2016;17(11):1558-1568.

11. Topalian SL, Hodi FS, Brahmer JR, et al. Safety, Activity, and Immune Correlates of Anti-PD-1 Antibody in Cancer. The New England journal of medicine. 2012;366(26):2443-2454 .

12. Brahmer JR, Tykodi SS, Chow LQM, et al. Safety and Activity of Anti-PD-L1 Antibody in Patients with Advanced Cancer. New England Journal of Medicine. 2012;366(26):2455-2465.

13. Boland PM, Ma WW. Immunotherapy for Colorectal Cancer. Cancers. 2017;9(5).

14. Overman MJ, Lonardi S, Wong KYM, et al. Durable Clinical Benefit With Nivolumab Plus Ipilimumab in DNA Mismatch Repair-Deficient/Microsatellite Instability-High Metastatic Colorectal Cancer. Journal of Clinical Oncology. 2018;36(8):773-779.

15. Ionov Y, Peinado MA, Malkhosyan S, Shibata D, Perucho M. Ubiquitous somatic mutations in simple repeated sequences reveal a new mechanism for colonic carcinogenesis. Nature. 1993;363(6429):558-561.

16. Sinicrope FA. DNA mismatch repair and adjuvant chemotherapy in sporadic colon cancer. Nature reviews Clinical oncology. 2010;7(3):174-177.

17. Hugo W, Zaretsky JM, Sun L, et al. Genomic and Transcriptomic Features of Response to Anti-PD-1 Therapy in Metastatic Melanoma. Cell. 2016;165(1):3544.

18. Shin DS, Zaretsky JM, Escuin-Ordinas H, et al. Primary Resistance to PD-1 Blockade Mediated by <em>JAK1/2</em> Mutations. Cancer Discovery. 2017;7(2):188-201. 
19. Ascierto ML, Makohon-Moore A, Lipson EJ, et al. Transcriptional Mechanisms of Resistance to Anti-PD-1 Therapy. Clinical Cancer Research. 2017;23(12):31683180.

20. Sade-Feldman M, Jiao YJ, Chen JH, et al. Resistance to checkpoint blockade therapy through inactivation of antigen presentation. Nature Communications. 2017;8(1):1136.

21. Jenkins RW, Barbie DA, Flaherty KT. Mechanisms of resistance to immune checkpoint inhibitors. British journal of cancer. 2018;118(1):1.

22. Gu T, De Jesus M, Gallagher HC, Burris TP, Egilmez NK. Oral IL-10 suppresses colon carcinogenesis via elimination of pathogenicCD4(+) T-cells and induction of antitumor CD8(+) T-cell activity. Oncoimmunology. 2017;6(6):e1319027.

23. Numasaki M, Fukushi J, Ono M, et al. Interleukin-17 promotes angiogenesis and tumor growth. Blood. 2003;101(7):2620-2627.

24. Cui G, Yuan A, Goll R, Florholmen J. IL-17A in the tumor microenvironment of the human colorectal adenoma-carcinoma sequence. Scandinavian journal of gastroenterology. 2012;47(11):1304-1312.

25. Al-Samadi A, Moossavi S, Salem A, et al. Distinctive expression pattern of interleukin-17 cytokine family members in colorectal cancer. Tumour biology : the journal of the International Society for Oncodevelopmental Biology and Medicine. 2016;37(2):1609-1615.

26. Tosolini M, Kirilovsky A, Mlecnik B, et al. Clinical impact of different classes of infiltrating T cytotoxic and helper cells (Th1, th2, treg, th17) in patients with colorectal cancer. Cancer Res. 2011;71(4):1263-1271.

27. Wu S, Rhee KJ, Albesiano E, et al. A human colonic commensal promotes colon tumorigenesis via activation of $\mathrm{T}$ helper type $17 \mathrm{~T}$ cell responses. Nature medicine. 2009;15(9):1016-1022. 
28. Wu P, Wu D, Ni C, et al. gammadeltaT17 cells promote the accumulation and expansion of myeloid-derived suppressor cells in human colorectal cancer. Immunity. 2014;40(5):785-800.

29. Housseau F, Wu S, Wick EC, et al. Redundant innate and adaptive sources of IL-17 production drive colon tumorigenesis. Cancer Res. 2016;76(8):2115-2124.

30. van de Kerkhof PC, Griffiths CE, Reich K, et al. Secukinumab long-term safety experience: A pooled analysis of 10 phase II and III clinical studies in patients with moderate to severe plaque psoriasis. Journal of the American Academy of Dermatology. 2016;75(1):83-98.e84.

\section{Chapter Five}

1. Manfredi S, Lepage C, Hatem C, Coatmeur O, Faivre J, Bouvier AM. Epidemiology and Management of Liver Metastases From Colorectal Cancer. Annals of surgery. 2006;244(2):254-259.

2. Wang CC, Li J. An update on chemotherapy of colorectal liver metastases. World journal of gastroenterology. 2012;18(1):25-33.

3. Bissahoyo A, Pearsall RS, Hanlon K, et al. Azoxymethane is a genetic background-dependent colorectal tumor initiator and promoter in mice: effects of dose, route, and diet. Toxicological sciences : an official journal of the Society of Toxicology. 2005;88(2):340-345.

4. Heijstek MW, Kranenburg O, Borel Rinkes IH. Mouse models of colorectal cancer and liver metastases. Digestive surgery. 2005;22(1-2):16-25.

5. Housseau F, Sears CL. Enterotoxigenic Bacteroides fragilis (ETBF)-mediated colitis in Min (Apc(+/-)) mice: A human commensal-based murine model of colon carcinogenesis. Cell cycle (Georgetown, Tex). 2010;9(1):3-5. 
6. Su LK, Kinzler KW, Vogelstein B, et al. Multiple intestinal neoplasia caused by a mutation in the murine homolog of the APC gene. Science (New York, NY). 1992;256(5057):668-670.

7. Hung KE, Maricevich MA, Richard LG, et al. Development of a mouse model for sporadic and metastatic colon tumors and its use in assessing drug treatment. Proceedings of the National Academy of Sciences. 2010;107(4):1565-1570.

8. Fu XY, Besterman JM, Monosov A, Hoffman RM. Models of human metastatic colon cancer in nude mice orthotopically constructed by using histologically intact patient specimens. Proceedings of the National Academy of Sciences of the United States of America. 1991;88(20):9345-9349.

9. Thalheimer A, Otto C, Bueter M, et al. The intraportal injection model: A practical animal model for hepatic metastases and tumor cell dissemination in human colon cancer. BMC Cancer. 2009;9(1):29.

10. Rashidi B, Sun FX, Jiang $P$, et al. A nude mouse model of massive liver and lymph node metastasis of human colon cancer. Anticancer research. $2000 ; 20(2 a): 715-722$.

11. Becker C, Fantini MC, Neurath MF. High resolution colonoscopy in live mice. Nature protocols. 2006;1(6):2900-2904.

12. Becker C, Fantini MC, Wirtz S, et al. In vivo imaging of colitis and colon cancer development in mice using high resolution chromoendoscopy. Gut. 2005;54(7):950-954.

13. Johnson RL, Fleet JC. Animal Models of Colorectal Cancer. Cancer metastasis reviews. 2013;32(0):39-61. 
APPENDICES 


\section{Appendix 1: APC $^{\text {min }}$ Genotyping Protocol}

1. Make a master mix of $150 \mu \mathrm{L}$ tail lysis buffer (Viagen DirectPCR (Tail), cat\# 102-

$\mathrm{T})$ per tail $+7.5 \mu \mathrm{L}$ proteinase $\mathrm{K}(20 \mathrm{mg} / \mathrm{mL})$ per tail.

2. Add $150 \mu \mathrm{L}$ master mix to a $1.5 \mathrm{~mL}$ Eppendorf tube containing each tail snip.

3. Digest in hybridization oven under constant rotation for 1 hour at $57^{\circ} \mathrm{C}$, vigorously shaking tubes halfway through digestion.

4. Inactivate proteinase $\mathrm{K}$ by placing tubes in $95^{\circ} \mathrm{C}$ water bath for 1 hour.

5. Create PCR master mix as follows:
a. Promega Go Taq Green 2x (6.75 $\mu \mathrm{L}$ per tail)
b. Notch1-F $(5 \mu \mathrm{M})(0.38 \mu \mathrm{L}$ per tail $)$
c. Notch1-R2 $(5 \mu \mathrm{M})(0.38 \mu \mathrm{L}$ per tail $)$
d. APC-M-C (5 $\mu \mathrm{M})(1.3 \mu \mathrm{L}$ per tail)
e. APC-M-M $(5 \mu \mathrm{M})(1.3 \mu \mathrm{L}$ per tail $)$
f. $25 \mathrm{mM} \mathrm{MgCl}_{2}(0.4 \mu \mathrm{L}$ per tail)

6. Add a total of $10.5 \mu \mathrm{L}$ of master mix to each well.

7. Add $2.5 \mu \mathrm{L}$ of DNA to each well (one tail per well).

8. Run PCR as follows:
a. Step $1: 94^{\circ} \mathrm{C} \times 10$ minutes
b. Step 2: $94^{\circ} \mathrm{C} \times 30$ seconds $(\times 33)$
c. Step $3: 53^{\circ} \mathrm{C}$ x 55 seconds
d. Step $4: 72^{\circ} \mathrm{C} \times 1$ minute
e. Step 5: $72^{\circ} \mathrm{C} \times 2$ minutes
f. $4^{\circ} \mathrm{C} \times \infty$

9. After PCR complete, pour $3 \%$ agarose gel

a. Mix $3 \mathrm{~g}$ agarose with $150 \mu \mathrm{L} 1 \times$ TBE buffer in a graduated cylinder 
b. Microwave for 2 minutes or until all agarose is dissolved and mixture is clear and boiling.

c. Add $2.5 \mu \mathrm{L}$ ethidium bromide to solution.

d. Pour into gel box.

e. Let cool completely (approximately 1 hour).

10. Load DNA ladder, negative control, positive control, tail samples into discrete wells.

11. Run gel at $150 \mathrm{~V}$ for 40 minutes or until adequate band separation occurs.

12. Image gel using ultraviolet transilluminator, $302 \mathrm{~nm}$.

13. Compare to representative image below to differentiate $A P C^{\min / /}$ from $A P C^{\min /+}$ mice.

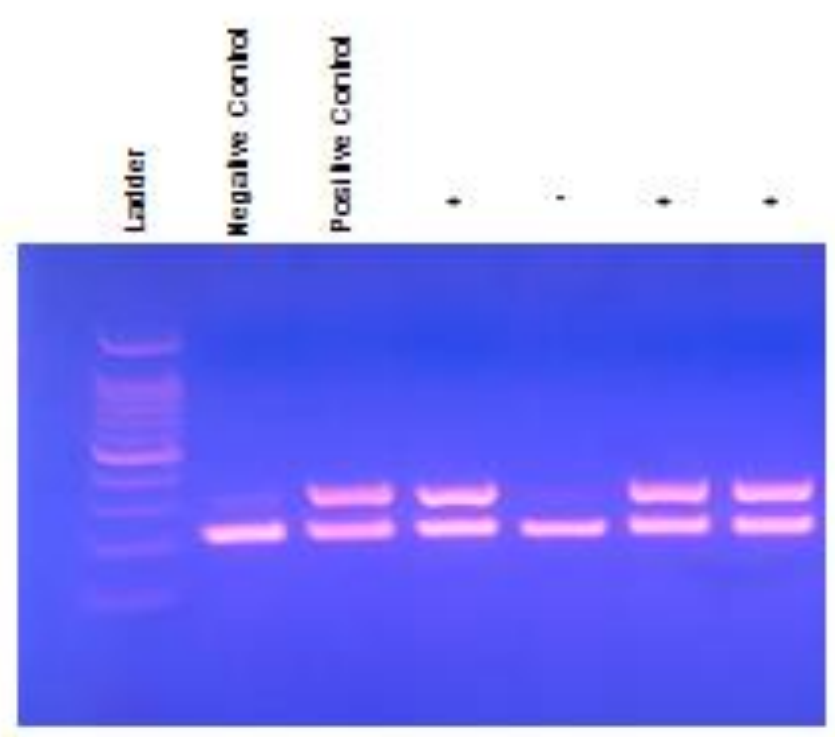




\section{Appendix 2: Enterotoxic Bacteroides fragilis (serotype 086) Growth Protocol}

\section{Preparing B. fragilis strain (086-ETBF) for inoculation}

1. The B. fragilis strains should be first brought up from the stock (usually stored in $30 \%$ glycerol at $-80^{\circ} \mathrm{C}$ ) by picking one loop of the stock using sterilized inoculation loop and streaking on pre-made BHI/Clindamycin agar plates. The inoculation agar plates then should be incubated at $37^{\circ} \mathrm{C}$ in an anaerobic condition for 2 days.

2. After the 2 days' incubation, take the agar plate out and exam the growth and the purity of bacteria. Pick a couple of colonies with pipette tip, swirl into a culture tube with $5 \mathrm{ml}$ of $\mathrm{BHI} / \mathrm{Clindamycin} \mathrm{broth}$. Cap the tube loosely and incubate at same condition as the agar plate above for $24-48$ hours.

3. Prepare a new tube with $10 \mathrm{ml}$ of $\mathrm{BHI} / \mathrm{Clindamycin}$ broth and sub-grow the bacteria

for 24 hours from the previous broth culture at a ratio of 1:100 dilution (inoculation $100 \mu \mathrm{l}$ of culture into $10 \mathrm{ml}$ of $\mathrm{BHI}$ broth).

4. After overnight incubation, the bacteria should grow well (the broth turn from clear to cloudy). Spin down bacteria (10,000rpm for 2 minutes), and wash the bacteria pellet twice with $1 \times$ PBS. Re-suspend into $1 \times$ PBS at $80 \%$ of the original volume, then adjust the OD600 $=0.6-0.8$.

\section{BHI Agar plates w/Hemin, Vitamin K, Clindamycin}

For 1 liter:

BHI: 37g/L (BD Bacto ${ }^{\mathrm{TM}}$ BHI, REF 237200) 
Yeast Extract: 5g/L (BD Bacto ${ }^{\mathrm{TM}}$, REF 212750)

L- Cysteine: 0.5g/L (Sigma C7352)

Agar: 15g/L (BD Bacto ${ }^{\mathrm{TM}}$, REF 214010)

- Combine ingredients in autoclavable container, add water to final volume of $1 \mathrm{~L}$

- $\quad$ Autoclave (sterilization time should be 30 minutes)

- Let broth cool down to room temperature, about 45 minutes

- $\quad$ Add:

Hemin solution: 10ml/L (Sigma H5533)

Vitamin K: $0.2 \mathrm{ml} / \mathrm{L}($ Sigma V3501)

Clindamycin: $6 \mu \mathrm{g} / \mathrm{mL}$

- Mix well by swirling the bottle. Do not shake the bottle (it will generate lots of bubbles)

- $\quad$ Pour plates; after BHI/Agar solution solidified, turn plates upside down, store plates in plastic bag at $4^{\circ} \mathrm{C}$.

\section{Liquid BHI broth}

BHI: 37g/L

Yeast Extract: $5 \mathrm{~g} / \mathrm{L}$

L- Cysteine: $0.5 \mathrm{~g} / \mathrm{L}$

- Combine ingredients in autoclavable container, add water to final volume of $1 \mathrm{~L}$

- $\quad$ Autoclave

- Let broth cool to room temperature

- $\quad$ Add: 
Hemin solution: $10 \mathrm{ml} / \mathrm{L}$

Vitamin $\mathrm{K}: \quad 0.2 \mathrm{ml} / \mathrm{L}$

Clindamycin: $6 \mu \mathrm{g} / \mathrm{mL}$

- Mix the solution

- $\quad$ Filter the broth, make aliquots, store at $4^{\circ} \mathrm{C}$ covered in silver foil.

\section{Hemin solution}

For $100 \mathrm{ml}$ :

- Weigh $50 \mathrm{mg}$ hemin in a weighing boat

- Add $1 \mathrm{ml}$ of $1 \mathrm{~N} \mathrm{NaOH}$ directly on the weighing boat, let it dissolve

- Add some $\mathrm{dH} 2 \mathrm{O}$

- Pour hemin solution into a $100 \mathrm{ml}$ bottle and add water to bring the volume to 100 $\mathrm{mL}$.

- Autoclave

- Store at $4^{\circ} \mathrm{C}$ covered in silver foil.

\section{Vitamin K1 solution}

- Dissolve $0.15 \mathrm{ml}$ of Vitamin $\mathrm{K} 1 \mathrm{in} 30 \mathrm{ml}$ of $95 \%$ ethanol

- Filter

- $\quad$ Store Vitamin $\mathrm{K} 1$ stock solution at $4^{\circ} \mathrm{C}$ in brown bottle or wrapped with aluminum foil. 


\section{Appendix 3: Enterotoxic Bacteroides fragilis (serotype 086) Inoculation Protocol}

1. Ensure mice are approximately 5 weeks of age. Younger mice will have a high mortality rate. In older mice, bacteria will be less effective at eliciting tumorigenesis.

2. 5 days prior to planned inoculation, place mice on water containing clindamycin $(0.1 \mathrm{~g} / \mathrm{L})$ and streptomycin $(5 \mathrm{~g} / \mathrm{L})$. Mice will take this water ad libitum until the day of inoculation.

3. On day of inoculation, place mice on standard water. Failure to do so will result in bacterial eradication after inoculation.

4. Using a 24 gauge feeding needle, administer $200 \mu \mathrm{L}$ ETBF suspension by oral gavage.

5. Inject each mouse with $500 \mu \mathrm{L}$ lactated ringers or normal saline intraperitoneally using a 26-30 gauge needle.

a. Mice suffer from dehydration as a result of the diarrhea that bacterial inoculation induces. IP fluid administration beginning on the day of ETBF inoculation helps prevent severe dehydration and decreases mouse mortality.

6. Monitor the mice daily for at least 7 days after bacterial inoculation. Administer $500 \mu \mathrm{L}$ lactated ringers or normal saline intraperitoneally as described in (5) throughout this time period.

7. For evaluation of early disease, begin treatment 7-10 days after ETBF inoculation. For evaluation of later stage disease, begin treatment 4 weeks after ETBF inoculation. 


\section{Appendix 4: Lymphocyte Isolation and Staining for Flow Cytometry Analysis}

\section{Protocol}

\section{Reagents}

- Pre-digestion Buffer

- $500 \mathrm{~mL} 1 \times$ PBS

○ $1 \mathrm{mM} \mathrm{DTT}(\mathrm{MW}=154.2,1 \mathrm{mM}=0.077 \mathrm{~g}$ in $500 \mathrm{~mL}$ )

- $10 \mathrm{mM}$ EDTA ( $\mathrm{MW}=372.2,10 \mathrm{mM}=1.861 \mathrm{~g}$ in $500 \mathrm{~mL}$ )

- Digestion Buffer (PREPARE FRESH PRIOR TO EACH USE)

○ 95 mL RPMl 1640

- $5 \mathrm{~mL}$ FBS

$\circ \quad 0.10 \mathrm{~g}$ Collagenase (Roche)

- Cell culture medium

- $500 \mathrm{~mL}$ RPMI 1640

○ $5 \%$ FBS

○ $100 \mathrm{U} / \mathrm{mL}$ penicillin $(50000 \mathrm{U} / 500 \mathrm{~mL})$

○ $100 \mathrm{U} / \mathrm{mL}$ streptomycin $(50000 \mathrm{U} / 500 \mathrm{~mL})$

- $2 \%$ FBS-PBS

- $90 \%$ Percoll (to be used in making $67 \%, 44 \%$ Percoll solutions)

- $90 \mathrm{~mL}$ Percoll

- $10 \mathrm{~mL} 10 x$ PBS

- $67 \%$ Percoll

○ $74.4 \mathrm{~mL} 90 \%$ Percoll

○ $25.6 \mathrm{~mL}$ cell culture medium

- $44 \%$ Percoll

○ $48.9 \mathrm{~mL} 90 \%$ Percoll 
- $\quad 51.1 \mathrm{~mL}$ cell culture medium

- FoxP3 Wash Buffer

- $5 \mathrm{~mL}$ FoxP3 wash buffer concentrate

○ $45 \mathrm{~mL} \mathrm{ddH} 20$

- Sample Fixing Buffer

- 250 microliters fixing buffer concentrate

○ 750 microliters fixing buffer diluent

- EC/IC Stain Master Mix

- 10 microliters FoxP3 wash buffer per sample

- $\quad 0.325-1.00$ microliters fluorophore-labeled antibody per sample

\section{Procedure}

1. Place Pre-digestion Buffer and Digestion Buffer in water bath at $37^{\circ} \mathrm{C}$ approximately 30 minutes prior to starting procedure.

2. Prepare ice tray for working with colon.

3. Sac mice, resect colon, mesenteric LN. Take care to remove excess mesenteric tissue from colon specimen, MLN.

4. Place MLN in 7-8 cc cell culture medium.

5. Lavage colon with ice cold PBS via plastic-tipped feeding needle to evacuate stool.

6. Hemisect colon longitudinally.

7. Place in petri dish with ice-cold PBS, wash vigorously for 10 seconds.

8. Morcellate very finely using scissors. Pieces should be between $0.2-0.5 \mathrm{~mm}$ in maximal diameter.

9. Place colon in $15 \mathrm{~mL}$ Pre-digestion Buffer.

10. Incubate colon at $37^{\circ} \mathrm{C}$ for 30 minutes in hybridization oven under slow rotation. 
For MLN Processing

11. Decant MLN + medium into small petri dish. Homogenize MLN between two frosted microscope slides.

12. Use $5 \mathrm{~mL}$ pipette to transfer cell suspension back to $15 \mathrm{~mL}$ centrifuge tube.

13. Spin $400 \mathrm{xg}, 4^{\circ} \mathrm{C}, 5 \mathrm{~min}$

14. Decant supernatant, resuspend with $10 \mathrm{~mL}$ media.

15. Transfer suspension into $50 \mathrm{~mL}$ centrifuge tube with 40 micron filter (green). Keep at 4 C.

For Colon Processing and LPL Isolation

16. Decant colon after incubation into labelled $50 \mathrm{~mL}$ centrifuge tube with 100 micron filter (yellow).

17. Add $10 \mathrm{~mL}$ room temperature $1 \times$ PBS to original centrifuge tube, decant into tube with filter.

18. Scrape tissue atop filter into original centrifuge tube, add $15 \mathrm{~mL}$ Digestion Buffer. Return to hybridization oven, incubate at $37^{\circ} \mathrm{C}$ for 30 minutes under slow rotation.

a. Note: This is a time-critical step. Not enough time will result in inadequate connective tissue digestion and poor LPL yield. Too much time will result in decreased cell viability.

19. After incubation, vortex each tube vigorously for 20 seconds.

a. Note: Vortexing for the full amount of time is critical to ensure connective tissue dissociation and liberation of LPL.

20. After vortexing, decant digested colon into labelled $50 \mathrm{~mL}$ centrifuge tube with 100 micron filter (yellow). 
21. Rub any remaining pieces of colon in the 100 micron filter gently to mechanically break up any residual connective tissue.

22. Add $10 \mathrm{~mL}$ cell culture medium to original $50 \mathrm{~mL}$ tube (the one in which the colon was digested), swirl, and decant into tube with 100 micron filter. This will collect any cells remaining in the tube/filter.

23. Centrifuge $L P L$ samples at $400 \mathrm{xg}, 4^{\circ} \mathrm{C}, 5 \mathrm{~min}$.

24. Discard supernatant and resuspend pellet in $10 \mathrm{~mL}$ cell culture medium. Decant into $50 \mathrm{~mL}$ centrifuge tube with 40 micron filter (green).

25. Centrifuge $L P L$ samples at $400 \mathrm{xg}, 4^{\circ} \mathrm{C}, 5 \mathrm{~min}$.

26. Discard supernatant. Resuspend pellet in $8 \mathrm{~mL} 44 \%$ Percoll (room temperature) and pipette cell suspension into $15 \mathrm{~mL}$ centrifuge tube.

27. Place glass Pasteur pipette $(1 \mathrm{~mL})$ in each centrifuge tube.

28. Slowly pipette $5 \mathrm{~mL} 67 \%$ Percoll (room temperature) into each Pasteur pipette to underlay the $67 \%$ Percoll under the cell suspension (cells $+44 \%$ Percoll). Be careful not to disturb sample and density gradient.

29. Centrifuge at $600 \mathrm{xg}, 2^{\circ} \mathrm{C}, 20$ mins. Make sure Accel $=\mathrm{SLOW}$, Decel $=$ OFF.

30. After slow spin, aspirate off supernatant above cell layer using transfer pipette. Get within $2 \mathrm{~mL}$ of cell layer (buffy coat) to remove as much fat and debris as possible.

31. Pipette cell layer (about 1-1.5 mL) into $10 \mathrm{~mL}$ cell culture in $15 \mathrm{~mL}$ centrifuge tube.

\section{Cell Stimulation}

32. Centrifuge at $400 \times \mathrm{g}, 4^{\circ} \mathrm{C}, 5$ mins for $\mathrm{LPL}, \mathrm{MLN}$. Discard supernatant and resuspend pellet in $2 \mathrm{~mL}$ cell culture medium for $\mathrm{LPL}, 10 \mathrm{~mL}$ cell culture medium for MLN. 
33. Count cells for each sample. Adjust cell concentration to 1-2 million per mL.

34. Add BFA (5 microliters per $\mathrm{mL}$ ), PMA (0.5 microliters per $\mathrm{mL}$ ), ionomycin (0.7 microliters per $\mathrm{mL}$ ), incubate at $37^{\circ} \mathrm{C}$ for $4-5$ hours.

\section{Staining for FACS Analysis}

35. After incubation, transfer samples to labeled flow tubes. Spin at 400xg, 3 mins, $4^{\circ} \mathrm{C}$.

36. Resuspend in 100 microliters cell culture medium. Add 0.7 microliters blocker (CD16/32) to each tube, incubate at room temperature $\times 8$ mins.

37. Make extracellular stain master mix

38. Add 10 microliters master mix per sample of EC stain master mix, incubate at room temperature $\times 12$ minutes

39. Centrifuge at $400 \times \mathrm{g}, 4^{\circ} \mathrm{C}, 3 \mathrm{~min}$.

40. Resuspend in $2 \mathrm{~mL} 2 \%$ FBS/PBS, centrifuge

41. Resuspend in 300 microliters per sample fixing buffer, incubate at $4^{\circ} \mathrm{C} \times 30 \mathrm{mins}$

42. Add 700 microliters FoxP3 wash buffer, centrifuge $1500 \mathrm{RPM}, 4^{\circ} \mathrm{C}, 3 \mathrm{~min}$

43. Make intracellular stain master mix

44. Resuspend in 50 microliters FoxP3 wash buffer. Add 10 microliters IC master mix per sample. Incubate overnight at $4^{\circ} \mathrm{C}$.

45. Add 700 microliters FoxP3 wash buffer, centrifuge $1500 \mathrm{RPM}, 4^{\circ} \mathrm{C}, 3 \mathrm{~min}$ 46. Reconstitute in 400 microliters FoxP3 wash buffer, ready for FACS analysis. 


\section{Appendix 5: APC ${ }^{\text {CKO }}$ (C57BL/6-Apctm1Tyj/J) Genotyping Protocol}

1. Make a master mix of $150 \mu \mathrm{L}$ tail lysis buffer (Viagen DirectPCR (Tail), cat\# 102-

$\mathrm{T})$ per tail $+7.5 \mu \mathrm{L}$ proteinase $\mathrm{K}(20 \mathrm{mg} / \mathrm{mL})$ per tail.

2. Add $150 \mu \mathrm{L}$ master mix to a $1.5 \mathrm{~mL}$ Eppendorf tube containing each tail snip.

3. Digest in hybridization oven under constant rotation for 1 hour at $57^{\circ} \mathrm{C}$, vigorously shaking tubes halfway through digestion.

4. Inactivate proteinase $\mathrm{K}$ by placing tubes in $95^{\circ} \mathrm{C}$ water bath for 1 hour.

5. Create PCR master mix as follows:
a. Promega Go Taq Green 2x (6.25 $\mu \mathrm{L}$ per tail)
b. Primer 1 (WT Fwd 01MR) $(5 \mu \mathrm{M})(1 \mu \mathrm{L}$ per tail)
c. Primer $2(\mathrm{WT}$ Rev 01MR) $(5 \mu \mathrm{M})(1 \mu \mathrm{L}$ per tail)
d. $\mathrm{ddH} 2 \mathrm{O}(2.25 \mu \mathrm{L}$ per tail)

6. Add a total of $10.5 \mu \mathrm{L}$ of master mix to each well.

7. Add $2.5 \mu \mathrm{L}$ of DNA to each well (one tail per well).

8. Run PCR as follows:
a. Step 1: $94^{\circ} \mathrm{C} \times 2$ minutes
b. Step 2: $94^{\circ} \mathrm{C} \times 25$ seconds
c. Step 3: $65^{\circ} \mathrm{C} \times 20$ seconds
d. Step $4: 68^{\circ} \mathrm{C} \times 15$ seconds
e. Repeat Steps $2-4 \times 10$ (11 cycles total)
f. Step 5: $94^{\circ} \mathrm{C} \times 20$ seconds
g. Step $6: 50^{\circ} \mathrm{C} \times 20$ seconds
h. Repeat Steps 5 and $6 \times 25$ (26 cycles total)
i. $4^{\circ} \mathrm{C} \times \infty$

9. After PCR complete, place samples in $-20^{\circ} \mathrm{C}$ overnight. 
10. The next morning, pour $3 \%$ agarose gel

a. Mix $3 \mathrm{~g}$ agarose with $150 \mu \mathrm{L} 1 \times$ TBE buffer in a graduated cylinder

b. Microwave for 2 minutes or until all agarose is dissolved and mixture is clear and boiling.

C. Add $2.5 \mu \mathrm{L}$ ethidium bromide to solution.

d. Pour into gel box.

e. Let cool completely (approximately 1 hour).

11. Load DNA ladder, negative control, positive control, tail samples into discrete wells.

12. Run gel at $150 \mathrm{~V}$ for 40 minutes or until adequate band separation occurs.

13. Image gel using ultraviolet transilluminator, $302 \mathrm{~nm}$.

14. Compare to representative image below to differentiate negative from positive mice.

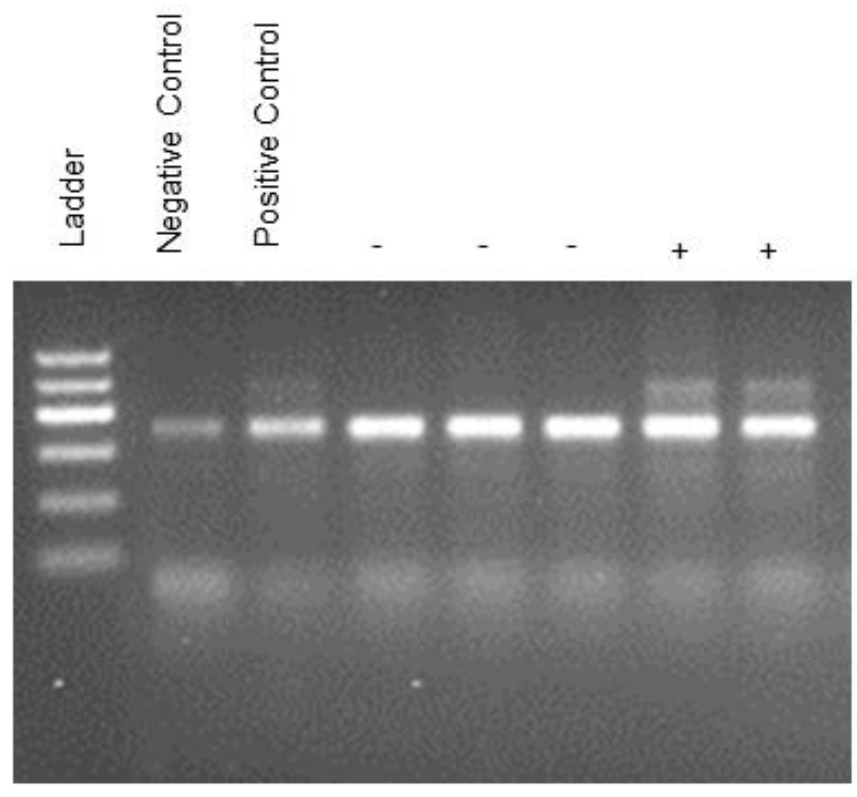




\section{Appendix 6: LSL-K-ras G12D (B6.129S4-Krastm4Tyj/J) Genotyping Protocol}

1. Make a master mix of $150 \mu \mathrm{L}$ tail lysis buffer (Viagen DirectPCR (Tail), cat\# 102-

$\mathrm{T})$ per tail $+7.5 \mu \mathrm{L}$ proteinase $\mathrm{K}(20 \mathrm{mg} / \mathrm{mL})$ per tail.

2. Add $150 \mu \mathrm{L}$ master mix to a $1.5 \mathrm{~mL}$ Eppendorf tube containing each tail snip.

3. Digest in hybridization oven under constant rotation for 1 hour at $57^{\circ} \mathrm{C}$, vigorously shaking tubes halfway through digestion.

4. Inactivate proteinase $\mathrm{K}$ by placing tubes in $95^{\circ} \mathrm{C}$ water bath for 1 hour.

5. Create PCR master mix as follows:
a. Promega Go Taq Green 2x (7.5 $\mu \mathrm{L}$ per tail)
b. Primer 1 (WT Fwd) $(5 \mu \mathrm{M})(1 \mu \mathrm{L}$ per tail)
c. Primer 2 (Mu Fwd) $(5 \mu \mathrm{M})(1 \mu \mathrm{L}$ per tail)
d. Primer 3 (Comm) $(5 \mu \mathrm{M})(1 \mu \mathrm{L}$ per tail)
e. $\mathrm{ddH} 2 \mathrm{O}(2.5 \mu \mathrm{L}$ per tail $)$

6. Add a total of $13.0 \mu \mathrm{L}$ of master mix to each well.

7. Add $2.0 \mu \mathrm{L}$ of DNA to each well (one tail per well).

8. Run PCR as follows:
a. Step 1: $94^{\circ} \mathrm{C} \times 2$ minutes
b. Step 2: $94^{\circ} \mathrm{C} \times 20$
c. Step 3: $65^{\circ} \mathrm{C} \times 15$ seconds
d. Step $4: 68^{\circ} \mathrm{C} \times 10$ seconds
e. Repeat Steps $2-4$ × 9 (10 cycles total)
f. Step 5: $94^{\circ} \mathrm{C} \times 15$ seconds
g. Step 6 : $60^{\circ} \mathrm{C} \times 15$ seconds
h. Repeat Steps 5 and $6 \times 27$ (28 cycles total)
i. $\quad 4^{\circ} \mathrm{C} \times \infty$ 
9. After PCR complete, pour $3 \%$ agarose gel

a. Mix $3 \mathrm{~g}$ agarose with $150 \mu \mathrm{L} 1 \times$ TBE buffer in a graduated cylinder

b. Microwave for 2 minutes or until all agarose is dissolved and mixture is clear and boiling.

c. Add $2.5 \mu \mathrm{L}$ ethidium bromide to solution.

d. Pour into gel box.

e. Let cool completely (approximately 1 hour).

10. Load DNA ladder, negative control, positive control, tail samples into discrete wells.

11. Run gel at $150 \mathrm{~V}$ for 40 minutes or until adequate band separation occurs.

12. Image gel using ultraviolet transilluminator, $302 \mathrm{~nm}$.

13. Compare to representative image below to differentiate negative from positive mice.

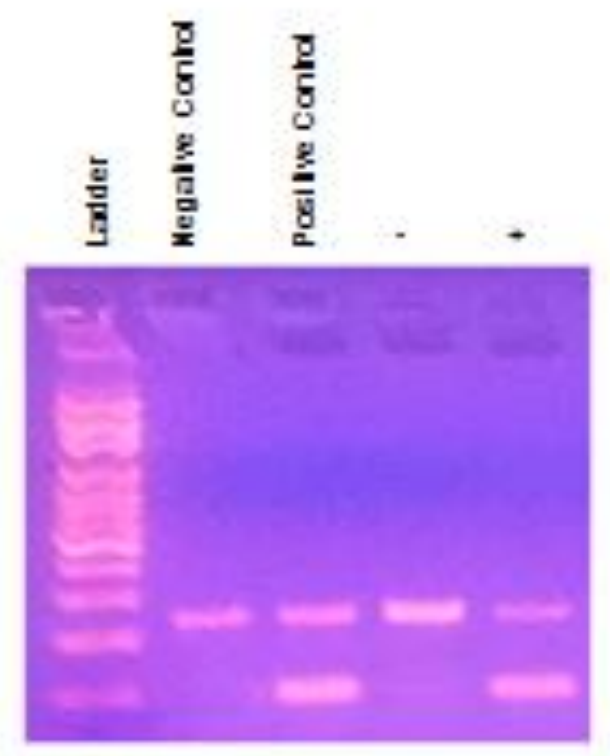




\section{Appendix 7: Publication Copyright Acknowledgements}

Chapter 1 was originally published in JNM. Bhutiani N, Grizzle WE, Galandiuk S, et al. Noninvasive Imaging of Colitis Using Multispectral Optoacoustic Tomography. Journal of nuclear medicine : official publication, Society of Nuclear Medicine. 2017;58(6):10091012. (C) SNMMI.

Chapter 3 has been accepted for publication in Oncoimmunology. Bhutiani $\mathbf{N}, \mathrm{Li}$ Q, Anderson CD, et al. Enhanced Gut Barrier Integrity Sensitizes Colon Cancer to Immune Therapy. Oncoimmunology. In press. 


\section{CURRICULUM VITAE}

Neal Bhutiani, MD

University of Louisville Department of Surgery

06 August 2018

550 S. Jackson St.

863-206-4008 (phone)

neal.bhutiani@louisville.edu

neal.bhutiani@gmail.com

\section{Personal Information}

Citizenship: United States of America

Date of Birth: 11 July 1987

Place of Birth: Winter Haven, Florida, USA

\section{Education}

2015-Present University of Louisville Department of Microbiology and Immunology

Ph.D. Candidate

2013-Present University of Louisville Department of Surgery

General Surgery Resident

2009-2013 Johns Hopkins University School of Medicine

M.D.

2005-2009 Duke University

B.S. in Biology and Political Science, summa cum laude

\section{Licensure}

Kentucky Board of Medical Licensure, \#R3156

\section{Certification}

Advanced Cardiac Life Support

Advanced Trauma Life Support

Honorary/Professional Societies

2017-Present Member, American Association for Cancer Research

2017-Present Resident Member, Society of Surgical Oncology 
2017-Present Trainee Member, American Association of Immunologists

2016-Present Member, American Association for the Advancement of Science

2016-Present Candidate Member, Association for Academic Surgery

2009

Phi Beta Kappa National Honor Society, Duke University

Pi Sigma Alpha National Honor Society, Duke University

\section{Invited Reviewer}

1. PLOS One (2018)

2. Surgery (2018)

3. Journal of Surgical Research (2017-Present)

\section{Language Skills}

Fluent in English and Spanish

\section{Publications}

$\underline{\text { Peer-reviewed Journals }}$

1. Warren SL, Bhutiani N, Agle SC, Martin RCG, 2nd, McMasters KM, Ajkay N. Differences between palpable and nonpalpable tumors in early-stage, hormone receptor-positive breast cancer. American journal of surgery. 2018.

2. Tierney J, Bhutiani N, Stamp B, Richey JS, Bahr MH, Vitale GC. Predictive risk factors associated with cholangitis following ERCP. Surgical endoscopy. 2018;32(2):799-804.

3. McClave SA, Patel J, Bhutiani N. Should fecal microbial transplantation be used in the ICU? Current opinion in critical care. 2018;24(2):105-111.

4. Jones JM, Bhutiani N, Wei D, Goldstein L, Jones CM, Cannon RM. A literaturebased cost analysis of tissue plasminogen activator for prevention of biliary stricture in donation after circulatory death liver transplantation. American journal of surgery. 2018.

5. Bhutiani N, Scoggins CR, McMasters KM, et al. The impact of caudate lobe resection on margin status and outcomes in patients with hilar cholangiocarcinoma: a multi-institutional analysis from the US Extrahepatic Biliary Malignancy Consortium. Surgery. 2018;163(4):726-731.

6. Bhutiani N, Mercer MK, Bachman KC, et al. Evaluating the Effect of Margin Consensus Guideline Publication on Operative Patterns and Financial Impact of Breast Cancer Operation. Journal of the American College of Surgeons. 2018.

7. Bhutiani N, Jones CM, Cannon RM, et al. Assessing relative cost of complications following orthotopic liver transplant. Clinical transplantation. 2018;32(4):e13209.

8. Bhutiani N, Egger ME, Ajkay N, Scoggins CR, Martin RC, 2nd, McMasters KM. Multigene Signature Panels and Breast Cancer Therapy: Patterns of Use and Impact on Clinical Decision Making. Journal of the American College of Surgeons. 2018;226(4):406-412.e401.

9. Bhutiani N, Adamson D, Eng M, Jones CM. An unusual case of cardiac failure following orthotopic liver transplantation. American journal of transplantation : official journal of the American Society of Transplantation and the American Society of Transplant Surgeons. 2018;18(4):1026-1028. 
10. Ajkay N, Bhutiani N, Huang B, et al. Early Impact of Medicaid Expansion and Quality of Breast Cancer Care in Kentucky. Journal of the American College of Surgeons. 2018;226(4):498-504.

11. Bhutiani N, Richey JS, Brown AN, et al. Assessing the value of endoscopic ultrasound in predicting symptom severity and long-term clinical course in chronic pancreatitis. HPB : the official journal of the International Hepato Pancreato Biliary Association. 2017;19(10):868-873.

12. Bhutiani N, Kimbrough CW, Burton NC, et al. Detection of microspheres in vivo using multispectral optoacoustic tomography. Biotechnic \& histochemistry : official publication of the Biological Stain Commission. 2017;92(1):1-6.

13. Bhutiani N, Grizzle WE, Galandiuk S, et al. Noninvasive Imaging of Colitis Using Multispectral Optoacoustic Tomography. Journal of nuclear medicine : official publication, Society of Nuclear Medicine. 2017;58(6):1009-1012.

14. Bhutiani N, Egger ME, McMasters KM. Optimizing Follow-up Assessment of Patients with Cutaneous Melanoma. Annals of surgical oncology. 2017;24(4):861-863.

15. Bhutiani N, Egger ME, Doughtie CA, et al. Intrapancreatic accessory spleen (IPAS): A single-institution experience and review of the literature. American journal of surgery. 2017;213(4):816-820.

16. Bhutiani N, Cheadle GA, Bahr MH, Vitale GC. Comparative Efficacy of Bilateral Thoracoscopic Splanchnicectomy for Intractable Pain Secondary to Pancreatic Cancer vs Chronic Pancreatitis. Journal of the American College of Surgeons. 2017;224(4):566-571.

17. Egger ME, Bhutiani N, Farmer RW, et al. Prognostic factors in melanoma patients with tumor-negative sentinel lymph nodes. Surgery. 2016;159(5):14121421.

18. Bhutiani N, Philips $P$, Scoggins CR, McMasters KM, Potts MH, Martin RC. Evaluation of tolerability and efficacy of irreversible electroporation (IRE) in treatment of Child-Pugh B (7/8) hepatocellular carcinoma (HCC). HPB : the official journal of the International Hepato Pancreato Biliary Association. 2016;18(7):593-599.

19. Bhutiani N, Philips P, Martin RC, 2nd, Scoggins CR. Impact of surgical margin clearance for resection of secondary hepatic malignancies. Journal of surgical oncology. 2016;113(3):289-295.

20. Bhutiani N, Martin RC, 2nd. Transarterial Therapy for Colorectal Liver Metastases. The Surgical clinics of North America. 2016;96(2):369-391.

21. Bhutiani N, Doughtie CA, Martin RC, 2nd. Ultrasound validation of mathematically modeled irreversible electroporation ablation areas. Surgery. 2016;159(4):1032-1040.

22. Bhutiani N, Akinwande $\mathrm{O}$, Martin RC, 2nd. Efficacy and Toxicity of Hepatic IntraArterial Drug-Eluting (Irinotecan) Bead (DEBIRI) Therapy in Irinotecan-Refractory Unresectable Colorectal Liver Metastases. World journal of surgery. 2016;40(5):1178-1190.

23. Bhutiani N, Agle S, Li Y, Li S, Martin RC, 2nd. Irreversible electroporation enhances delivery of gemcitabine to pancreatic adenocarcinoma. Journal of surgical oncology. 2016;114(2):181-186.

24. Bender M, Pradilla G, Batra S, et al. Effectiveness of repeat glycerol rhizotomy in treating recurrent trigeminal neuralgia. Neurosurgery. 2012;70(5):1125-1133; discussion 1133-1124.

25. Bhutiani N, Martin RCG II. Irreversible Electroporation for Treatment of Locally Advanced Pancreatic Cancer. CSurgeries. 2016. 
26. Cameron EH, Bhutiani N, Bozeman MC. Will Availability of SMOF Lipid Emulsions for Parenteral Nutrition Change Surgical Nutrition Practice? Current Nutrition Reports. In press.

27. Bhutiani N, Graffree B, Martin RCG II, Quillo AR. Factors influencing intraoperative parathyroid hormone levels and correlation with curative treatment of primary hyperthyroidism. The American Surgeon. In press.

28. Bhutiani N, Philips P, Martin RCG, Scoggins CR, McMasters KM, Davis EG, Bahr MH, Vitale GC, Bozeman, MC. Correlation of Biliary Colic in the Absence of Cholelithiasis with Pancreaticobiliary Obstruction. The American Surgeon. In press.

29. Bhutiani N, Miller KR, Benns MV, Smith JW, Harbrecht BG, Bozeman MC. Correlating Geographic Location with Incidence of Motor Vehicle Induced Pedestrian Injury. The American Surgeon. In Press.

30. Brown SR, Bhutiani N, Quillo AR. Optimizing Follow-up in Post-operative Parathyroidectomy Patients. The American Surgeon. In press.

31. Rostas JW, Bhutiani N, Crigger M, Crawford S, Hollenbach R, Heidrich S, Martin RCG, McMasters KM, Ajkay N. Calculation of Breast Volumes from Mammogram: Comparison of Four Separate Equations Relative to Mastectomy Specimen Volumes. Journal of Surgical Oncology. In press.

32. Bhutiani N, Schucht JE, Miller KR, McClave SA. Technical Aspect of Fecal Micribiome Transplant. Current Gastroenterology Reports. In press.

33. Hegde BN, Bhutiani N, Bozeman MC. Use of parenteral nutrition to manage malignant obstruction. Current Surgery Reports. In press.

34. Venegas-Borsellino CP, Sonikpreet, Bhutiani N. Nutritional Therapy in Neuro Oncology. Current Surgery Reports. In press.

35. Ludwig NA, Bhutiani N, Linsky PL, Dwivedi AJ; Bozeman MC. Outcomes and follow-up in patients undergoing endovacular repair for traumatic thoracic aortic injury. The American Surgeon. In press.

36. Bhutiani N, Quinn, SA, Jones JM, Mercer MK, Farmer RW, Scoggins CR, McMasters KM, Martin RCG. Financial impact of ERAS protocol implementation on perioperative care for upper gastrointestinal and HPB malignancies. Surgery. In press.

37. Bhutiani N, Li Q, Anderson CD, Gallagher HC, De Jesus M, Singh R, Jala VR, Fraig M, Gu T, Egilmez NK. Enhanced Gut Barrier Integrity Sensitizes Colon Cancer to Immune Therapy. Oncoimmunology. In press.

38. Martin EK, Bhutiani N, Martin RCG. Safety and efficacy of irreversible electroporation (IRE) for treatment of hilar cholangiocarcinoma. Presented (mini oral) at AHPBA 2018. HPB. In press.

39. Bhutiani N, Egger ME, Stromberg AJ, Gershenwald JG, Ross MI, Martin RCG II, Scoggins CR, McMasters KM. A model for predicting non sentinel lymph node status in patients with sentinel lymph node positive cutaneous melanoma. Journal of Surgical Oncology. In press.

\section{Book Chapters}

1. Bhutiani N, Benns MV, Miller KR. "Surgical Alteration of the Gastro-Intestinal Tract, Inherent Complications, and Implications for Nutrition Support."

A.S.P.E.N. Adult Nutrition Support Core Curriculum $3^{\text {rd }}$ Edition. 
2. Bhutiani N, Martin RCG II. "Hepatic Arterial Embolization Therapies." Primary and Metastatic Liver Tumors: Treatment Strategy and Evolving Therapies. In Press

3. Bhutiani N, Jones CM. Ex-vivo Resection/Transplantation for Hepatic Neoplasms. Society of Surgical Oncology Surgical Council on Resident Education (SCORE) Module.

\section{$\underline{\text { Abstracts }}$}

1. Hong YK, Pandit H, Pulliam Z, Bhutiani N, Li S, Li Y, Martin RCG. Enhancing immunotherapy by epigenetic silencing of histone and DNA methylation in pancreatic cancer. Society of Surgeons of the Alimentary Tract 2017 Annual Meeting.

2. Hong YK, Shah J, Kim W, Bhutiani N, Stevenson M, Egger ME, Philips P, McMasters KM, Martin RCG, Scoggins CR. Primitive neuroectodermal tumor (PNET) incidence, treatment patterns, and outcomes: analysis of the National Cancer Database 2004 to 2014. Southwestern Surgical Congress 2018 Annual Meeting.

3. Bhutiani N, Pandit H, Zheng Q, Li S, Li Y, Martin RCG. Electrochemotherapy with irreversible electroporation and FOLFIRINOX improves survival in murine pancreatic adenocarcinoma by increasing apoptosis and decreasing tumor cell proliferation. AACR 2018 Annual Meeting, Society of Surgical Oncology Annual Cancer Symposium 2018.

4. Bhutiani N, Wei D, Patkar AD, Johnston S, Martin RCG. Cost analysis of complications following pancreatic resection. Pancreas Club 2018 Annual Meeting.

5. Mercer MK, Bhutiani N, Ajkay N. Changing perceptions and practices regarding post-operative lymphedema in breast cancer. 2018 American Society of Breast Surgeons Annual Meeting.

6. Bruenderman EH, Bhutiani N, Mercer MK, Ajkay N. Evaluation of the presence of calcifications at the margins of operative specimens in patients with DCIS. Society of Surgical Oncology Annual Cancer Symposium 2018.

7. Bhutiani N, Quinn SA, Farmer RW, Scoggins CR, McMasters KM, Martin RCG. Financial impact of ERAS protocol implementation on perioperative care for upper gastrointestinal and HPB malignancies. Central Surgical Association 2018 Annual Meeting.

8. Bhutiani N, Mortensen GF, Brown AN, Bahr MH, Martin RCG, Vitale GC. Technical aspects of successful ERCP and stenting pre- and post- IRE in patients with unresectable pancreatic adenocarcinoma. Americas HepatoPancreato-Biliary Association Annual Meeting 2018.

9. Bhutiani N, Scoggins CR, McMasters KM, Martin RCG. A treatment algorighm for management of neuroendocrine liver metastases. Americas HepatoPancreato-Biliary Association Annual Meeting 2018.

10. Martin EK, Bhutiani N, Martin RCG. Safety and efficacy of irreversible electroporation (IRE) for treatment of hilar cholangiocarcinoma. Americas Hepato-Pancreato-Biliary Association Annual Meeting 2018.

11. Tierney J, Bhutiani N, Brown AN, Richey JS, Bahr MH, Vitale GC. Identifying factors predicting response to endoscopic management of chronic pancreatitis secondary to pancreas divisum. Society of American Gastrointestinal and Endoscopic Surgeons Annual Meeting 2018. 
12. Mortensen GF, Bhutiani N, Brown AN, Palin H, Richey JS, Tierney J, Vitale GC. Safety and efficacy of ERCP in patients with chronic abdominal pain in the setting of non-dilated duct disease. Society of American Gastrointestinal and Endoscopic Surgeons Annual Meeting 2018.

13. Bhutiani N, Jones JM, Wei D, Goldstein LJ, Jones CM, Cannon RM. Cost analysis of post-operative biliary strictures in patients undergoing orthotopic liver transplantation. 2018 American Transplant Congress.

14. Bhutiani N, Quinn SA, Mercer MK, Hong YK, Stevenson M, Egger ME, Philips P, Martin RCG, Scoggins CR. Identifying risk factors for peri-operative VTE in patients undergoing resection for gastrointestinal malignancy. 2018

Southwestern Surgical Congress Annual Meeting.

15. Bhutiani N, Harbrecht BG, Scoggins CR, Bozeman MC. Assessing the early impact of Medicaid expansion on operative care for benign gallbladder disease in Kentucky. 2018 Southwestern Surgical Congress Annual Meeting.

16. Bhutiani N, Egger ME, Stromberg AJ, Bhutiani IK, Ajkay N, McMasters KM. Identifying Factors Impacting Efficacy of Postmastectomy Radiotherapy in Women with T1-2 Breast Cancer and 1-2 Positive Lymph Nodes. 2018 Society of Surgical Oncology Symposium.

17. Bhutiani N, Egger ME, Ajkay N, Stromberg AJ, Scoggins CR, Martin RCG II, McMasters KM. Multigene Signature Panels and Breast Cancer Therapy: Patterns of Utilization and Impact on Clinical Decision-Making. 2017 Southern Surgical Association Annual Meeting.

18. Ajkay N, Bhutiani N, Huang B, Howard JD, Scoggins CR, McMasters KM, Polk $\mathrm{HC}$ Jr. Evaluating the Early Impact of Medicaid Expansion on the Quality of Breast Cancer Care in Kentucky. 2017 Southern Surgical Association Annual Meeting.

19. Bhutiani N, Mercer MK, Bachmann K, Heidrich SR, Martin RCG II, Scoggins CR, McMasters KM, Ajkay N. Evaluating the effect of margin consensus guideline implementation on operative patterns and financial impact of breast cancer surgery. 2017 Western Surgical Association Annual Meeting.

20. Bhutiani N, Raque JL, Mercer MK, May H, Donaldson M, Hines K, McMasters KM, Ajkay N. The Effect of Regional Anesthesia on Post-Operative Narcotic Use in Patients Undergoing Mastectomy for Breast Cancer. 2017 Meeting of the Kentucky Chapter of the American College of Surgeons.

21. Hong YK, Pandit H, Bhutiani N, Li S, Li Y, Martin RC II. Enhancing Immunotherapy by Epigenetic Silencing of Histone and DNA Methylation in Pancreatic Cancer. Society for Surgery of the Alimentary Tract 2017 Annual Meeting.

22. Mercer MK, Bhutiani N, Rostas JW, Ajkay N. Estimated Percentage Of Breast Volume Excision And Its Relationship With Quality Of Life And Satisfaction After Breast Conservation Therapy For Breast Cancer. 2017 American Society of Breast Surgeons Annual Meeting.

23. Bhutiani N, Miller KR, Benns MV, Smith JW, Harbrecht BG, Bozeman MC. Correlating Geographic Location with Incidence of Motor Vehicle Induced Pedestrian Injury. 2017 Annual Meeting of the Southwestern Surgical Congress

24. Bhutiani N, Li Q, Anderson CD, Gu T, Egilmez NK. Combined Oral Cytokine Therapy Effectively Treats Colon Cancer in a Murine Model. 2017 AACR Annual Meeting

25. Bhutiani N, Sobolev A; Fouts B; Chuong P; McNally M; Khanal B; Khanal A; Neal M; McNally LR. pH Specific Dual Targeting of Colloidal Mesoporous Silica Nanoparticles for Pancreatic Adenocarcinoma. 2017 AACR Annual Meeting 
26. Bhutiani N, Grizzle WE, Egilmez NK, Dryden GW, Galandiuk S, McNally LR. Non-invasive Imaging of Colitis using Multispectral Optoacoustic Tomography. 2017 AACR Annual Meeting.

27. Bhutiani N, Richey J, Bahr M, Vitale GC. Correlation of endoscopic ultrasound (EUS) findings with natural history and progression of disease in patients with chronic pancreatitis. Americas Hepato-Pancreato-Biliary Association Annual Meeting 2017.

28. Bhutiani N, Philips P, Scoggins CR, McMasters KM, Martin RCG. Assessing Relative Cost of Complications following Hepatic Resection. Americas HepatoPancreato-Biliary Association Annual Meeting 2017.

29. Bhutiani N, Philips P, Scoggins CR, McMasters KM, Martin RCG. Assessing Relative Cost of Complications following Pancreatic Resection. Americas Hepato-Pancreato-Biliary Association Annual Meeting 2017.

30. Bhutiani N, Philips P, Scoggins CR, McMasters KM, Martin RCG. Assessing Relative Cost of Complications following Orthotopic Liver Transplantation. Americas Hepato-Pancreato-Biliary Association Annual Meeting 2017.

31. Tierney, J; Bhutiani N; Stamp B; Richey J; Egger ME; Brown A; Bahr MH; Vitale GC. Predicting the risk of post-ERCP cholangitis. 2017 SAGES Annual Meeting.

32. Bhutiani N; Egger ME; Stromberg A; Quillo AR; Martin RCG II; Scoggins CR; McMasters KM. A logistic regression based calculator for predicting non sentinel lymph node positivity in patients with sentinel lymph node positive cutaneous melanoma. 2017 Society of Surgical Oncology Symposium.

33. Bhutiani N, Philips P, Scoggins CR, McMasters KM, Martin RCG. Assessing Relative Cost of Complications following Bile Duct Surgery. 2017 Society of Surgical Oncology Symposium.

34. Bhutiani N, Philips P, Scoggins CR, McMasters KM, Martin RCG. Assessing Relative Cost of Complications following Hepatic Ablation. 2017 Society of Surgical Oncology Symposium.

35. Brown SR; Bhutiani N; Quillo AR. Optimizing Follow-up in Post-operative Parathyroidectomy Patients. 2017 Academic Surgical Congress.

36. Edwards KN; Bhutiani N; Quillo AR. Assessing the influence of Graves' Disease on the incidence of thyroid cancer. 2017 Academic Surgical Congress.

37. Rostas JW, Bhutiani N, Philips P. Local advancement flap vs. skin graft in complex melanoma resection. 2017 Academic Surgical Congress.

38. Bhutiani N, Cheadle J; Stanelle E; Guzman J; Bahr M; Vitale GC. Comparing the efficacy of bilateral thoracoscopic splanchnicectomy for pain relief in patients with pancreatic cancer and chronic pancreatitis. 2016 Annual Meeting of the Southern Surgical Association.

39. Bhutiani $\mathbf{N}$, et al. The impact of caudate resection for hilar cholangiocarcinoma on margin status and outcome: results from the United States Extrahepatic Biliary Consortium. 2016 Annual Meeting of the Western Surgical Association.

40. Bhutiani N, Agle SC, Li Y, Li S, Martin RCG II. "Irreversible Electroporation Enhances Delivery of Gemcitabine to Pancreatic Adenocarcinoma." 2016 Meeting of the Kentucky Chapter of the American College of Surgeons.

41. Bhutiani N, Philips P, Scoggins CR, McMasters KM, Potts MH, Martin RCG. "Evaluation of Tolerability and Efficacy of Irreversible Electroporation (IRE) in Treatment of Child-Pugh B (7/8) Hepatocellular Carcinoma (HCC)." $12^{\text {th }}$ World Congress of the International Hepato-Pancreatico-Biliary Association.

42. Bhutiani N, Graffree B, Martin RCG II, Quillo AR. Factors influencing time to decrease in intraoperative parathyroid hormone levels in patients undergoing focused parathyroidectomy. 2016 Academic Surgical Congress. 
43. Bhutiani N, Doughtie CA, Martin RC II. Ultrasound validation of mathematically modeled irreversible electroporation ablation areas. 2016 Academic Surgical Congress.

44. Pradilla G, Bhutiani N, Batra S, Bender M, See AP, Garzon-Muvdi T, James C, Carson BS, Lim M. 2011. Safety and efficacy of secondary microvascular decompression as treatment for recurrent trigeminal neuralgia. College of Neurological Surgeons 2011 Annual Meeting.

\section{Presentations}

Oral

1. Bhutiani N, Quinn SA, Mercer MK, Hong YK, Stevenson M, Egger ME, Philips $P$, Martin RCG, Scoggins CR. "Identifying risk factors for peri-operative VTE in patients undergoing resection for gastrointestinal malignancy." 2018 Southwestern Surgical Congress Annual Meeting. Napa, CA. April 2018.

2. Bhutiani N, Harbrecht BG, Scoggins CR, Bozeman MC. "Assessing the early impact of Medicaid expansion on operative care for benign gallbladder disease in Kentucky." 2018 Southwestern Surgical Congress Annual Meeting. Napa, CA. April 2018.

3. Bhutiani N, Egger ME, Stromberg AJ, Bhutiani IK, Ajkay N, McMasters KM. "Identifying Factors Impacting Efficacy of Postmastectomy Radiotherapy in Women with T1-2 Breast Cancer and 1-2 Positive Lymph Nodes." Society of Surgical Oncology Annual Cancer Symposium 2018. Chicago, IL. March 2018.

4. Bhutiani N, Pandit H, Zheng Q, Li S, Li Y, Martin RCG. "Electrochemotherapy with irreversible electroporation and FOLFIRINOX improves survival in murine pancreatic adenocarcinoma by increasing apoptosis and decreasing tumor cell proliferation." Society of Surgical Oncology Annual Cancer Symposium 2018. Chicago, IL. March 2018.

5. Bhutiani N, Quinn SA, Farmer RW, Scoggins CR, McMasters KM, Martin RCG. "Financial impact of ERAS protocol implementation on perioperative care for upper gastrointestinal and HPB malignancies." Central Surgical Association 2018 Annual Meeting. Columbus, OH. March 2018.

6. Bhutiani N, Mortensen GF, Brown AN, Bahr MH, Martin RCG, Vitale GC. "Technical aspects of successful ERCP and stenting pre- and post- IRE in patients with unresectable pancreatic adenocarcinoma." Americas HepatoPancreato-Biliary Association Annual Meeting 2018. Miami, FL. March 2018.

7. Bhutiani N, Scoggins CR, McMasters KM, Martin RCG. "A treatment algorighm for management of neuroendocrine liver metastases." Americas HepatoPancreato-Biliary Association Annual Meeting 2018. Miami, FL. March 2018.

8. Bhutiani N, Raque JL, Mercer MK, May H, Donaldson M, Hines K, McMasters KM, Ajkay N. "The Effect of Regional Anesthesia on Post-Operative Narcotic Use in Patients Undergoing Mastectomy for Breast Cancer." 2017 Meeting of the Kentucky Chapter of the American College of Surgeons. Lexington, KY. September 2017.

9. Bhutiani N, Philips P, Scoggins CR, McMasters KM, Martin RCG. "Assessing Relative Cost of Complications following Orthotopic Liver Transplantation." 2017 AHPBA Annual Meeting, Miami, FL. March-April 2017.

10. Bhutiani N, Egger ME, Stromberg AJ, Quillo AR, Martin RCG II, Scoggins CR, McMasters KM. "A model for predicting non sentinel lymph node poisitivity in 
patients with sentinel lymph node positive cutaneous melanoma." 2017 Society of Surgical Oncology Symposium, Seattle, WA. March 2017.

11. Bhutiani N, Cheadle J; Stanelle E; Guzman J; Bahr M; Vitale GC. "Comparing the efficacy of bilateral thoracoscopic splanchnicectomy for pain relief in patients with pancreatic cancer and chronic pancreatitis." 2016 Annual Meeting of the Southern Surgical Association, Palm Beach, FL. December 2016

12. Bhutiani N, et al. "The impact of caudate resection for hilar cholangiocarcinoma on margin status and outcome: results from the United States Extrahepatic Biliary Consortium." 2016 Annual Meeting of the Western Surgical Association, Coronado, CA. November 2016

13. Bhutiani N, Agle SC, Li Y, Li S, Martin RCG II. "Irreversible Electroporation Enhances Delivery of Gemcitabine to Pancreatic Adenocarcinoma." 2016 Meeting of the Kentucky Chapter of the American College of Surgeons. Lexington, KY. August 2016

14. Bhutiani N, Philips P, Scoggins CR, McMasters KM, Potts MH, Martin RCG. "Evaluation of Tolerability and Efficacy of Irreversible Electroporation (IRE) in Treatment of Child-Pugh B (7/8) Hepatocellular Carcinoma (HCC)." $12^{\text {th }}$ World Congress of the International Hepato-Pancreatico-Biliary Association, Sao Paolo, Brazil. April 2016.

15. Bhutiani N, Graffree B, Martin RCG II, Quillo AR. "Factors influencing time to decrease in intraoperative parathyroid hormone levels in patients undergoing focused parathyroidectomy." $11^{\text {th }}$ Annual Academic Surgical Congress, Jacksonville, FL. February 2016.

16. Bhutiani N, Doughtie CA, Martin RCG. "Ultrasound Validation of Mathematically Modeled Irreversible Electroporation (IRE) Ablation Areas." $11^{\text {th }}$ Annual Academic Surgical Congress, Jacksonville, FL. February 2016.

\section{$\underline{\text { Poster }}$}

1. Bhutiani N, Pandit H, Zheng Q, Li S, Li Y, Martin RCG. "Electrochemotherapy with irreversible electroporation and FOLFIRINOX improves survival in murine pancreatic adenocarcinoma by increasing apoptosis and decreasing tumor cell proliferation." AACR 2018 Annual Meeting, Chicago, IL. April 2018

2. Bhutiani N, Sobolev A, Fouts B, Chuong P, McNally M, Khanal B, Khanal A, Neal M, McNally LR. "pH Specific Dual Targeting of Colloidal Mesoporous Silica Nanoparticles for Pancreatic Adenocarcinoma." 2017 AACR Annual Meeting, Washington, DC. April 2017

3. Bhutiani N, Li Q, Anderson CD, Gu T, Egilmez NK. Combined Oral Cytokine Therapy Effectively Treats Colon Cancer in a Murine Model. 2017 AACR Annual Meeting, Washington, DC. April 2017.

4. Bhutiani N, Philips P, Scoggins CR, McMasters KM, Martin RCG. "Assessing Relative Cost of Complications following Hepatic Resection." 2017 AHPBA Annual Meeting, Miami, FL. March-April 2017.

5. Bhutiani N, Philips P, Scoggins CR, McMasters KM, Martin RCG. "Assessing Relative Cost of Complications following Pancreatic Resection." 2017 AHPBA Annual Meeting, Miami, FL. March-April 2017.

6. Bhutiani N, Philips P, Scoggins CR, McMasters KM, Martin RCG. "Assessing Relative Cost of Complications following Bile Duct Surgery." 2017 Society of Surgical Oncology Symposium, Seattle, WA. March 2017. 
7. Bhutiani N, Philips P, Scoggins CR, McMasters KM, Martin RCG. "Assessing Relative Cost of Complications following Hepatic Ablation." 2017 Society of Surgical Oncology Symposium, Seattle, WA. March 2017.

8. Bhutiani N, Egilmez NK, McNally LR. "Tracking uptake and biodistribution of fluorophore containing nanoparticles using multispectral optoacoustic tomography (MSOT)." 2016 World Molecular Imaging Congress, New York, NY. September 2016.

9. Pradilla G, Bhutiani N, Batra S, Bender M, See AP, Garzon-Muvdi T, James C, Carson BS, Lim M. 2011. "Safety and efficacy of secondary microvascular decompression as treatment for recurrent trigeminal neuralgia." College of Neurological Surgeons 2011 Annual Meeting. Washington, DC.

10. Bhutiani, N. Characterizing Lactate Consumption in Rat Mammary Adenocarcinoma (R3230) Cells. Duke University Biology Honors Thesis Symposium. May 2009.

\section{Research Funding}

1. Bhutiani $\mathrm{N}(\mathrm{PI})$. Evaluting donor leukocyte telomere length as a predictor of time to decreased allograft function in solid organ transplant. Kentucky Organ Donation Affiliates (KODA). \$20,000: 2017

2. Bhutiani $\mathrm{N}$ (Co-investigator). The effect of irreversible electroporation on delivery and efficacy of gemcitabine and FOLFIRINOX in murine models of pancreatic adenocarcinoma. University of Louisville School of Medicine Basic Grant. \$20,000: 2016-2017

\section{Invited Speaking Engagements}

1. Managing Personal Finances. Invited panel discussant. $3^{\text {rd }}$ Annual Surgeons as Scholars and Leaders Consortium. Jackson, WY. January 2017

2. Exercise in the ICU. Invited lecture. Kentucky Society of Parenteral and Enteral Nutrition Annual Meeting. Louisville, KY. June 2017. 